
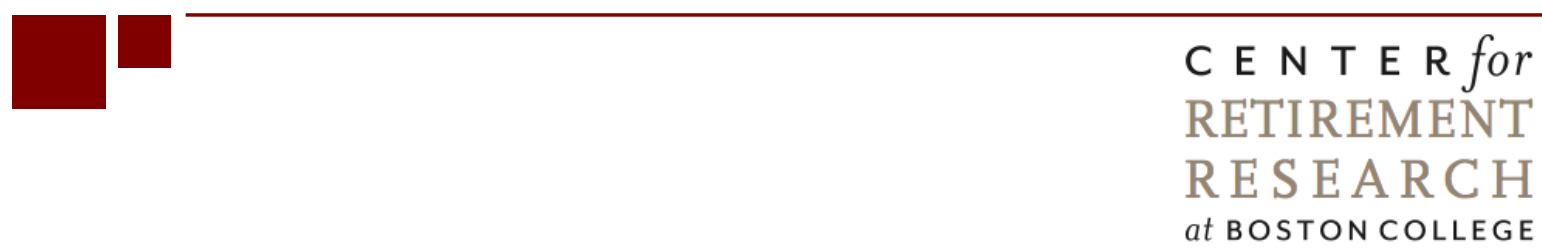

\title{
THE EQUILIBRIUM AND SPILLOVER EFFECTS OF EARLY RETIREMENT
}

\author{
Simon Jäger, Benjamin Schoefer, and Josef Zweimüller \\ CRR WP 2020-3 \\ January 2020 \\ Center for Retirement Research at Boston College \\ Hovey House \\ 140 Commonwealth Avenue \\ Chestnut Hill, MA 02467 \\ Tel: 617-552-1762 Fax: 617-552-0191 \\ https://crr.bc.edu
}

Simon Jäger is an assistant professor of economics at the Massachusetts Institute of Technology. Benjamin Schoefer is an assistant professor of economics at the University of California, Berkeley. Josef Zweimüller is a professor of economics at the University of Zurich. The research reported herein was performed pursuant to a grant from the U.S. Social Security Administration (SSA) funded as part of the Retirement Research Consortium. The opinions and conclusions expressed are solely those of the authors and do not represent the opinions or policy of SSA, any agency of the federal government, the Massachusetts Institute of Technology, the University of California, Berkeley, the University of Zurich, or Boston College. Neither the United States Government nor any agency thereof, nor any of their employees, makes any warranty, express or implied, or assumes any legal liability or responsibility for the accuracy, completeness, or usefulness of the contents of this report. Reference herein to any specific commercial product, process or service by trade name, trademark, manufacturer, or otherwise does not necessarily constitute or imply endorsement, recommendation or favoring by the United States Government or any agency thereof. The authors would like to thank Alberto Abadie, Daron Acemoglu, Josh Angrist, David Autor, Mark Bils, Peter Hull, Philipp Kircher, Patrick Kline, Moritz Kuhn, Guido Menzio, as well as audiences at conferences at briq Workshop on Jobs, Firms and Inequality, London-Paris Public Economics conference, CEPR/IZA Annual Symposium in Labour Economics, CEPR Public Economics Symposium, MILLS Workshop 2019, MunichWorkshop on Natural Experiments, NBER Summer Institute Macro Perspectives Meeting, NBER Labor Studies Meeting, NBER Public Economics Meeting, NBER Aging Program Meeting, NBER Longer Working Lives and Labor Demand Workshop, SED Meeting, CESifo Venice Summer Institute, and at seminars at Banca d'Italia, Harvard, IAB Colloquium, INSEAD, the Federal Reserve Bank of Philadelphia, UC Berkeley, UC Santa Barbara, and University of Pennsylvania. Karl Aspelund, Carolin Baum, Nikhil Basavappa, Dominik Egloff, David Hughes, Andreas Kettemann, René Livas, Damian Osterwalder, Nina Roussille, Philippe Ruh, Martina Uccioli, and Samuel Young provided excellent research assistance.

(C) 2020, Simon Jäger, Benjamin Schoefer, and Josef Zweimüller. All rights reserved. Short sections of text, not to exceed two paragraphs, may be quoted without explicit permission provided that full credit, including (C) notice, is given to the source. 


\begin{abstract}
About the Steven H. Sandell Grant Program
This paper received funding from the Steven H. Sandell Grant Program for Junior Scholars in Retirement Research. Established in 1999, the Sandell program's purpose is to promote research on retirement issues by scholars in a wide variety of disciplines, including actuarial science, demography, economics, finance, gerontology, political science, psychology, public administration, public policy, sociology, social work, and statistics. The program is funded through a grant from the Social Security Administration (SSA). For more information on the Sandell program, please visit our website at: http://crr.bc.edu/?p=9570, send e-mail to crr@bc.edu, or call (617) 552-1762.
\end{abstract}

\title{
About the Center for Retirement Research
}

The Center for Retirement Research at Boston College, part of a consortium that includes parallel centers at the National Bureau of Economic Research, the University of Michigan, and the University of Wisconsin-Madison, was established in 1998 through a grant from the U.S. Social Security Administration. The Center's mission is to produce first-class research and forge a strong link between the academic community and decision-makers in the public and private sectors around an issue of critical importance to the nation's future. To achieve this mission, the Center sponsors a wide variety of research projects, transmits new findings to a broad audience, trains new scholars, and broadens access to valuable data sources.

Center for Retirement Research at Boston College

Hovey House

140 Commonwealth Ave

Chestnut Hill, MA 02467

Tel: 617-552-1762 Fax: 617-552-0191

https://crr.bc.edu

\author{
Affiliated Institutions: \\ The Brookings Institution \\ Mathematica - Center for Studying Disability Policy \\ Syracuse University \\ Urban Institute
}




\begin{abstract}
This paper examines the labor market effects of unemployment insurance extensions. It uses administrative Social Security matched employer-employee data from Austria. Critical components of the analysis are effects on wages as well as retirement/job separation effects.

The paper found that:

- Older workers are very responsive to unemployment insurance benefit extensions, which in our setting may have served as a bridge into early retirement.

- These separations stem from high-value employment relationships, and may constitute inefficient separations.

- Wages appear unresponsive to such shifts on workers' nonemployment outside options, implying that these workers may not be able to use such reforms to improve their bargaining power - even if they are older and on the retirement margin.

- However, in a second study, we find no effects on separations of such policies even for older workers. The key difference is that one reform was potential benefit extensions (entailing early retirement) rather than the benefit levels (in increases in the generosity of which even older workers did not separate from their jobs).
\end{abstract}

The policy implications of the findings are:

- Unemployment insurance benefit generosity may interact with retirement policies and trigger early retirement among older workers eligible for these benefits.

- These separations may be socially inefficient and may therefore have welfare costs.

- Subsidizing early retirement policies does not entail wage pressure. 


\section{Introduction}

By Coasean theories of jobs, the employer and the worker exploit all bilateral gains from trade and reach bilaterally efficient outcomes, through flexible bargaining and contracting. Hence all job separations are mutually preferable and efficient, in that no bargain could have been found, however complex, to prevent the separation and save the employment relationship. By contrast, frictional ("non-Coasean") settings put limits on such contracting, for example in form of wage rigidity or institutional constraints. In such settings, inefficient separations can emerge. Whichever of these two perspectives provides a better description of real-world job dynamics naturally determines the welfare properties of employment adjustment and hence the potential scope for policy interventions. Yet, an empirical test of the Coasean benchmark is challenging exactly because of the abstract allocative concepts underlying its strong efficiency properties in the first place: joint job surplus split by unrestricted transferable-utility compensation arrangements 1

We present an empirical evaluation of whether the Coasean theory of jobs can account for separation behavior. Since surplus and bargaining cannot be directly observed, we implement a revealed-preference test. We study a transitory treatment that, while active, reduces job surplus and thereby causes separations. After the treatment is sharply deactivated, the group of surviving jobs then lacks a mass of marginal (low-surplus) matches. ${ }^{2}$ These surviving jobs should exhibit resilience to any kind of subsequent shocks - compared to a control group never exposed to the initial treatment, as the sole statistic determining separations is joint job surplus (with wage bargaining rendering the initial incidence of shocks on the firm or worker side irrelevant).

Our specific treatment reducing job surplus is a nonemployment subsidy, specifically a program implemented in Austria in 1988 that raised the duration of unemployment insurance benefits from one to four years. Since eligibility was determined by age cutoffs (age 50 and up) and was restricted to specific regions, we implement a difference-in-differences design comparing age groups and regions, in the universe of Austrian social security data. The program was suddenly abolished in 1993, providing us with the opportunity to implement our revealed preference test: after the program abolition, the group of formerly treated job survivors, whom we track in population-level administrative panel data, should be more resilient - have fewer separations - in response to any future shocks, compared to their control peers.

Our first empirical step documents that the program triggered an increase in separations of $10.9 \mathrm{ppt}(27 \%)$ over the five-year horizon the program was active: $51.4 \%$ of jobs in the treatment group separated, compared to a counterfactual separation rate of $40.5 \%$ in the absence of the reform 3

\footnotetext{
${ }^{1}$ For example, measured flow wages, even if appearing rigid, can be viewed as consistent with bilaterally efficient bargaining outcomes (Barro, 1977, Hall, 2005: Shimer. 2004: Hall and Milgrom, 2008), and labels like quit and layoff can both denote efficient separations (McLaughlin, 1991).

${ }^{2}$ Our point of departure are theories featuring heterogeneity in match surplus (as in, e.g., Mortensen and Pissarides. 1994: Bils, Chang, and Kim, 2012: Mui and Schoefer 2019), in contrast to simplified models of perfect homogeneity, either on the worker side (e.g. Hansen. 1985| Rogerson |1988), or on the firm (productivity) side as in textbook DMP models without heterogeneity (Pissarides. 2000).

salive et al. (2015) study the same program with a focus on search externalities from treated onto untreated
} 
The efficiency benchmark predicts that these program-induced separations should have extracted low joint surplus jobs. This low surplus property is not directly measurable by observables. To study the attributes of these incremental separators, we extend the complier analysis method by Imbens and Rubin (1997) and Abadie (2003) to difference-in-differences settings such as ours. These incremental separators did not carry features that would map clearly into surplus or efficiency properties. They only exhibit somewhat lower worker fixed effects, are more likely to stem from blue-collar jobs, and from industries with high disability incidence, which are characteristics perhaps pointing towards low worker surplus - but, for example, the compliers have similar wages and many other attributes as the other groups.

In a second step, we implement our revealed-preference strategy. We exploit the abrupt abolition of the policy in 1993, in our panel data tracking the job survivors (among those jobs active already at the onset of the program in 1988) in both the treatment and control groups. By the Coasean benchmark, the abolition should realign the surplus distributions among job survivors: each surviving control group job has an active, iso-surplus peer in the former treatment group, except, crucially, for a missing mass of marginal matches, which separated and which we do not track among the survivors in our panel data. This set of marginal jobs should have surplus between zero and a cutoff equal to the surplus value of the UI program, and are the first to separate ahead of any inframarginal program survivors.

Strikingly, in the data, the two groups exhibit identical post-abolition separation behavior despite the massive depletion in the ranks of the former treatment group, which is around 18\% smaller than the control group due to the treatment. The lack of resilience in separations holds unconditionally as well as in response to negative labor demand shocks (sharp establishment shrinkage events and negative industry growth).

Juxtaposed, the large separations effect and subsequent absence of resilience are hard to reconcile with the Coasean view of jobs. Specifically, we show that the Coasean view can only rationalize the perfect comovement of separation rates under narrow conditions: if idiosyncratic job-level surplus exhibits no persistence whatsoever, so that the post-abolition surplus distribution immediately and perfectly reconverges between the former treatment and control groups, despite the large extraction of marginal jobs. Yet, such perfect reshuffling may be implausible, as it would need to occur within the very first year after the program abolition.

To quantify this rationalization formally within the Coasean framework, we estimate the weight the data put on either a full-persistence benchmark or an immediate-and-perfect-reshuffling benchmark. We estimate this model structurally with GMM, using variation across cohorts, and industryoccupation cells. Point estimates imply that $100 \%$ (lower end of $95 \%$ confidence interval: $89.0 \%$ ) of

unemployed job seekers' job finding rates; Inderbitzin et al. (2016) study effects on disability insurance entry among the unemployed, but also assess composition effects from the inflow margin. Winter-Ebmer (2003) studies inflow effects of the program between two broad groups (all below 50 vs. 50-65) using a $\%$ sample of our population data. Lalive et al. (2011) find small inflow effects of different, national reform in Austria. Kyyrä and Wilke (2007) provide empirical evidence on the interaction of UI and retirement in Finland. Hartung et al. (2018) study the macro consequences of a German UI reform, including separations. Feldstein (1976) and Doornik et al. (2018) study how UI eligibility affects temporary layoffs and recalls. 
jobs would need to follow the "full-reshuffling already within one year post-abolition" benchmark for a Coasean model to rationalize the data.

In this estimation, we exploit the model-implied piece-wise linear relationship between the treatment group separations and the control group separations after the policy's abolition. We assume that the groups are hit by the same aggregate surplus shocks within cells across the regions. In the Coasean model, separations follow a single-dimensional "pecking order" of jobs ranked by joint job surplus. Our first benchmark is given by a model variant with perfect persistence in idiosyncratic job surplus. Then, for small shocks, separations occur in the control group but not in the treatment group - up until a "kink" beyond which the former treatment group should start seeing separations. That kink is positioned at the job that was marginal with respect to the UI extension in the model, and in the data its position is identified as the control group reaching a post-abolition separation rate corresponding to the treatment effect from the initial UI extension. The treatment effect was large, so that kink is far to the right of zero on the x-axis, and hence this full-persistence benchmark predicts substantial resilience among the program survivors. Our second benchmark is a variant in which job-level surplus has no persistence whatsoever. Here, the previous extraction of marginal jobs by the policy is inconsequential for the post-abolition surplus distribution, which immediately converges across the two groups. As a result, the former treatment group post-abolition separations move one-to-one with control group separations. That is, they are predicted to trace out the 45 -degree line (as we find in the data), rather than the piecewise linear kinked relationship with the resilience (zero-separations) region (as predicted by the the aforementioned persistence variant).

Our preferred alternative interpretation of the absence of resilience is that the separations were not Coasean (rather than surplus reshuffling within a short period of time). Yet, while all efficient separations are alike, each frictional setting is inefficient in its own way, as with Tolstoy's unhappy families. A specific non-Coasean candidate that is parsimonious and perhaps particularly plausible for our sample builds on wage-setting frictions, for example completely fixed wages on the job 4 Then, exactly by preventing the flexible (re)bargaining underlying the Coasean result, such frictions complicate the "positive surplus" test from single-dimensional Coasean joint surplus, to two unilateral surplus concepts: separations may occur because either worker-specific or firm-specific surplus turns negative. As a result, jobs can inefficiently separate even though one party's surplus remains large enough to, in principle, bribe the other party into continuation (if only the requisite trade were implementable).

In this non-Coasean setting, the original UI extension, which boosted worker's outside options, would have destroyed matches with initially low worker surplus - but not necessarily with low firm surplus. After the abolition, the non-Coasean model would therefore predict resilience in response to worker-surplus shocks - but analogously not necessarily to firm surplus shifts. Consequently, if firm-surplus shocks account for most post-abolition separation, then the non-Coasean model

\footnotetext{
${ }_{4}^{4}$ Jager et al. (2018) document that Austrian wages appear unresponsive to UI benefit level shifts (studying reforms that did not entail separations). We cannot credibly study wage effects in the present empirical setting given the large attrition implied by the separation effects.
} 
can rationalize the empirical perfect comovement of post-abolition separations between former treatment and control groups - even if idiosyncratic job-level surplus has persistence. In fact, the non-Coasean model naturally suggests a direct reinterpretation of the previous structural estimation of the Coasean one. Now, the perfect empirical comovement we document implies that at least $89 \%$ of the post-abolition separations are due to low firm surplus.

This non-Coasean narrative is consistent with, first, the absence of resilience specifically in response to negative labor demand shocks (sharp establishment shrinkage events and negative industry growth). Second, for our sample of older workers, the implied type of joint distribution of high worker surplus and low firm surplus turns out to be predicted by the long-standing hypothesis of implicit contract models, in the form of backloading of compensation over the job spell (Lazear. 1979. 1981): in a period-by-period consideration, young (low-tenure) workers are "underpaid", while older (high-tenure) workers are "overpaid.' 5 In fact, the Austrian institutional setting features an explicit role for works councils that are consulted in the separation process, providing formal support for such implicit contracts. Third, the tentative evidence on the characteristics of the marginal jobs we document in our complier analysis appears somewhat consistent with this narrative on some dimensions, such as larger separation effects in industries with a higher share of sick or disability leave.

We also resolve an ostensible tension: why did the extraction of so many low worker-surplus jobs in response to the initial UI program not measurably lower separation rates of the survivors? The resolution has two simple features. First, high typical worker surplus rationalizes why few workers are usually on their unilateral separation margin. Indeed, our sample consists of male, older, and high-tenured workers. Institutionally, Austria mandates multiple months of severance payments in the case of layoffs that are potentially foregone when quitting, providing little incentive for workers to quit unilaterally and pushing up worker surplus. Second, the exceptional generosity of the UI extension - four years of eligibility, potentially serving as a bridge into early retirement, and worth around $70 \%$ of an annual salary - still strongly affected those typical workers otherwise inframarginal to normal worker-side shocks. 6

Section 2 presents an overview of the Austrian institutional context, the policy, and our data. In Section 3, we document the large separation effects entailed by the UI extension. We also conduct a complier analysis to characterize the marginal jobs destroyed by the policy. In Section 4. we study the separation behavior of the treatment and control groups after the program was abolished. Section 5 assesses the capacity of the Coasean model to rationalize the data, while Section 6 presents a specific non-Coasean setting that can potentially do so. Section 7 concludes.

F Frimmel et al. (2018) shed light on this hypothesis studying seniority-wage profiles and separations. Employer competition model with downward wage rigidity (Cahuc et al. 2006) generate an increasing wage-experience profile with high worker surplus and small firm surplus, but these models feature bilaterally efficient bargaining and separations.

${ }^{6}$ A prediction of this view is that smaller shifts in outside options should not induce workers to separate. Indeed,Jäger et al. (2018), who study wage responses in response to UI benefit changes from different Austrian reforms, document that such smaller benefit shifts did not entail separation effects even among older workers and even during the 1980s in Austria. 


\section{Institutional Context, the Policy Variation, and Data}

We review the UI system, the UI reform, other relevant institutional context as well as our data below.

\subsection{The Austrian UI System and the UI Benefit Extension}

The Austrian UI System During the 1980s and 1990s Two crucial institutional features ensure that UI generosity cleanly shifts the nonemployment outside option of workers in our setting. First, Austrian workers are fully eligible for UI benefits upon quitting, after fulfilling a four-week waiting period. Second, similar to most other European countries, the Austrian system does not feature experience rating, as the Austrian UI system is funded through employer and employee payroll taxes (which were not affected by the reform).

During the 1980s and 1990s, the gross replacement rate was between 40 and $48 \%$ for most employees, and capped below and above at a minimum and maximum amount.7 The potential benefit duration (PBD) of UI benefits during the 1980s was 30 weeks, provided the worker had worked (and paid contributions to the UI system) for at least three out of the last five years; otherwise, PBD was 20 weeks. After exhaustion of UI, the unemployed can apply for unemployment assistance (UA, "Notstandshilfe"). UA benefits are means-tested (on income of other household members) and granted for successive 39-week periods, but could in principle be extended forever if eligibility is maintained. UA benefits are capped at 92 percent of UI benefits; in 1990 the median paid UA was about 70 percent of the median UI benefit. 59 [26] percent of the unemployed receive UI [UA].

1988-93 Regional Extended Benefit Program (REBP) In 1988, the Austrian government enacted a regional extended benefit program (going forward "REBP"), a large region- and age-specific expansion of potential benefit duration - originally 30 weeks - to 209 weeks for older (50+) workers in a subset of regions in the country. We calculate the cash value of extended benefits to correspond to about $71 \%$ of a typical worker's annual salary in Appendix A Figure 1 Panel (a) presents the resulting potential benefit duration (PBD) by age group over time.

A job loser had to satisfy each of the following criteria at the beginning of his or her unemployment spell: (i) age 50 or older; (ii) a continuous work history (780 employment weeks during the last 25 years prior to the current unemployment spell); (iii) residence in any of the 28 selected labor market districts for at least 6 months prior to the claim; and (iv) start of a new unemployment spell after June 1988 or spell in progress in June 1988.8

Therefore, the reform induces variation along multiple dimensions, permitting a difference-indifferences design: first, across age as we can compare workers aged 50 and above to their younger

${ }^{7} \mathrm{UI}$ benefits are not taxed. The net replacement ratio, UI benefits over the wage net of social security contributions and income taxes, is around 55\%, slightly higher than in the US. See Jäger et al. (2018) for details on replacement rates.

${ }^{8}$ The location criterion was further tightened by a 1991 reform which added the requirement to also have had employment in the REBP regions. 
peers; second, across regions: REBP regions to non-REBP regions 9 The difference-in-differences nature of our research design allows us to control for unobservable confounders at the region and age/cohort level. We net out region-level shocks (including market-level effects of the reform) by comparing slightly younger and older workers in the same region who were narrowly affected or unaffected by the reform. We also net out age- or cohort-specific factors by comparing the same cohorts across regions.

Figure 1 Panel (a) also plots an economy-wide UI reform in August 1989, again based on age and experience. 10 That national 1989 reform raises PBD for workers aged 40 to 49 [50 and above] to 39 [52] weeks with an experience requirement of 312 [468] weeks of employment in the last 10 [15] years. This 1989 reform is orthogonal to the regional variation we analyze: as it applied uniformly across all regions, it is netted out through our cross-regional differences. More precisely, then, for job losers from August 1989 onward, REBP's incremental effect on duration was by 3 years (as then the baseline PBD was 52 weeks), and it was 3.44 years (= 209 weeks - 30 weeks) before August 1989.

The policy aimed to mitigate labor market consequences of a crisis in the iron-, steel- and other heavy industries ("steel sector" in the following). The state-owned company, the Oesterreichische Industrie AG (OeIAG), had suffered from low commodity prices, shrinking markets, and low productivity since the mid-1970s. In response, the new OeIAG management implemented a sequence of restructuring plans during the 1980s, leading to plant closures and downsizing. The REBP regions were selected due to their large share of employment in the steel sector: in the REBP regions, about $17 \%$ of workers were employed in the steel sector, compared to around $5 \%$ in the non-REBP-regions. Figure 1 Panel (b) provides a map of the REBP labor market districts.

Importantly, REBP did not impose any industry requirement for a job loser's eligibility: all unemployed who met criteria (i) to (iv) were eligible. To minimize UI policy endogeneity problems, our empirical analysis below excludes workers employed in the steel sector. We address any further concerns that remaining workers may be subject to spillovers from the steel sector decline or otherwise to differential regional shocks or trends by capturing - and netting out - such effects with our second difference of young ineligible cohorts in the REBP and non-REBP regions.

Abolition of the Program REBP was initially in effect until December 1991 before it was extended in January 1992 11 REBP was then abolished (stopped accepting new entrants) on August 1, 1993, though claimants e.g. in ongoing spells who established eligibility to REBP before August 1993

\footnotetext{
${ }^{9}$ In principle, we could leverage additional variation over time and using an experience cutoff for the reform. In Section 3.1. we describe why we focus on region and cohorts as our main source of variation.

${ }^{10}$ The reform also increased the replacement rate from 41 to $47 \%$ in the monthly income bracket from 5,000 to 10,000 ATS, roughly 400 to 800 USD at the time.

${ }^{11}$ The 1992 extension enacted two changes for new spells. First, the benefit extension was abolished in 6 of the original 28 regions. We exclude from our analysis the set of treated regions that were excluded after the 1991-reform. Second, the 1992-extension tightened eligibility criteria for extended benefits: new beneficiaries had to be not only residents, but also previously employed in a treated region.
} 
continued to be covered 12 The abolition decision was formally announced in June 1993, an implementation gap of only two months. The program end occurred in an abrupt fashion: in fact, in January 1993 the Austrian government had come up with a plan to expand access to longer benefit duration to older unemployed workers in all Austrian regions from one to four years along with changes in the eligibility requirements 13 In the following weeks and months, the government reversed course completely and abolished REBP.

\subsection{Other Institutional Details}

Interaction of UI with Other Social Policies Interacting with other welfare programs, REBP could effectively serve as a bridge into permanent nonemployment. In the absence of the REBP, unemployed men could effectively retire early at age 58 by claiming unemployment benefits for one year and special income support for another year before drawing a regular public pension at age 60 (the retirement age for male workers with at least 35 years of social security insurance contributions) ${ }^{14}$ Since the REBP extended the maximum duration of UI benefits by three years, eligible workers could already permanently withdraw from the labor force at age 55.

Another important program was disability insurance (DI). During the study period, the Austrian system granted relaxed access to a DI pension from age 55 onward, allowing job losers in REBP regions to retire already as young as age 51 while being on some kind of benefit up until age 60 , when they could then start claiming their public pension. ${ }^{15}$

Advance Notice for Layoffs, Works Councils, and Severance Pay While employment protection is not as stringent as in many other countries, an Austrian firm laying off a worker has to obey a set of rules. At the time of the REBP, the firm had to give advance notice, which amounted to 5 [ 4 , $3,2,1.5]$ months for workers with at least $25[15,5,2,0]$ years of tenure. Workers, too, are obliged to give a (one-month) advance notice.

\footnotetext{
${ }^{12}$ In addition, the 1993 abolition featured a grandfathering clause (\$81) that granted longer benefits for those employment relationships that had already been announced to be terminated before the implementation of the reform, but for which the advance notice period would lead to a formal end of the relationship after the abolition date. We describe the advance notice periods below. Empirically, we analyze post-REBP resilience starting in 1994q1 to take account of the grandfathering clauses.

${ }^{13}$ We confirm this course of events in a newspaper content analysis. For instance, Der Standard (a major Austrian newspaper) reported in an article entitled "Länger Geld für alle Altersarbeitslosen (Longer benefits for all unemployed workers)" from January 9, 1993: "All older unemployed workers throughout Austria - and not only in [REBP regions] as in the past - will be eligible for unemployment benefits of four years instead of one. Minister of Social Affairs, Josef Hesoun, and the social partners have agreed in principle on this [...]." [translation by the authors].

${ }^{14}$ For men, our sample, special income support is a program available for unemployed workers during the last year before they can claim the regular public pension. For workers with long contribution histories, the statutory retirement age was 60 (unemployed women aged 59) during the study period. Special income support was therefore available at age 59. Special income support is equivalent to UI spell in legal terms, but with 25 percent higher benefits, paid for a period of at most 12 months.

${ }^{15} \mathrm{DI}$ applicants below age 55 get a DI pension when a health impairment reduces the work capacity by more than 50 percent in any occupation. In contrast, DI applicants above age 55 are considered as disabled if their work capacity is lower than 50 percent in the same occupation. In practice, this means that not only health but also employability criteria establish DI access after age 55.
} 
The Austrian labor law provides a role for works councils in the layoff process. In firms with 5 or more employees, workers can organize in works councils. The firm has to inform and consult the works council when a layoff is planned and, if the firms fails to do so, the layoff becomes void. If a layoff violates substantial interests of the worker, the firm has to prove that the layoff is economically necessary for the survival of the firm. The works council must also be consulted when choosing the particular worker to be separated. The works council also ensures that potential hardships of layoffs candidates are taken into account, which provides some employment protection for older and longer-tenured workers. Mass layoffs in larger firms are subject to specific further rules. Firms with more than 100 employees that reduce employment by more than 5 percent (or more than 50 employees) within one month must give written notice to the regional employment agency, one month before the mass layoff is implemented, where failure to notify renders the mass layoff void.

In case of a layoff, the firm has to make a severance payment to the worker in our study period. The amount is a step function of worker tenure: $3(5,10,15,20,25)$ years of tenure map into $2(3$, $4,6,9,12)$ monthly salaries, and zero below three years. Severance payments are only due for the following separation types: layoffs (but not dismissals for cause), job terminations upon mutual agreement (between firm and worker), and after the end of a temporary contract. In contrast, worker-induced quits are exempt from the severance-pay rule, except for workers with more than 10 years of tenure.

\subsection{Data and Sample}

The Austrian Social Security Database (ASSD) is a matched employer-employee data set covering the universe of private-sector and non-tenured public sector employees in Austria from 1972 onward (Zweimüller et al., 2009). 16

We drop all individuals working in the steel sector because the reform targeted these workers who presumably face worse labor market prospects. Likewise, we drop the 6 regions that were covered by the REBP only until 1991. We also drop women for data and institutional reasons 17 The vast majority of our sample fulfilled the experience requirement; since this sample restriction turned out to not affect our estimates, we present the unconditional results. We report summary statistics for our main analyses in Table 1. We primarily focus on workers born after 1933 as older cohorts had already exited the REBP program at the time of its abolition due to regular retirement. We include cohorts up to workers born in 1948, so that our age-based control cohorts are those born between 1943 and 1948, who were younger than 50 at the time the program was abolished in 1993.

\footnotetext{
${ }^{16}$ We complement the ASSD with data from the Austrian Ministry of Social Affairs on employment histories before 1972, to determine whether or not a worker is eligible for REBP (which is based on work experience within the last 25 years).

${ }^{17}$ First, whereas old age insurance rules allow men to retire at age 60, women can retire at age 55. Second, individuals must have been employed in 15 out of the last 25 years in order to be eligible for REBP. Since we cannot observe all 25 years prior to the reform, it is likely that classification errors arise for women, who have a less continuous work history than men in our data.
} 


\section{Large Separation Effects from the REBP Unemployment Insurance Benefit Extension}

In Section 3.1. we estimate that the differential benefit extension (from 52 to 209 weeks) increased job separations among eligible workers by 10.9ppt (27\%) among initial matches over the five year program horizon, in the treated cohorts compared to their ineligible peers. Most of these excess separations went into long-term nonemployment perhaps followed by early retirement, rather than to employment with other firms. We present visual evidence of raw data before turning to regression estimates, to assess the parallel trends assumptions underlying our research design. In Section 3.2. we additionally characterize the incremental REBP separators in a complier analysis, showing that it would be difficult to assess the efficiency properties of these separations on the basis of observables, motivating our subsequent revealed-preference strategy on the basis of postabolition separation behavior of job survivors in Section 4 .

\subsection{Separation Effects}

Plotting Raw Data: Cohort Gradients of Separations We sort the population of 1988 job holders (the onset of the reform) by month-of-birth cohorts and into REBP and non-REBP regions, and then plot outcomes over the course of REBP in Figures 2 and 3 . Each figure presents levels for each cohort by region, and the within-cohort, between-region difference.

Younger cohorts born after 1943 turned 50 after the REBP was abolished in 1993 and therefore could never claim extended benefits under the program. Older cohorts born before 1933, while eligible for extended benefits, were older than 55 at the time the REBP was initiated in 1988 and, at that age, also had access to more generous disability/early retirement benefits with relaxed entry conditions, as described in Section 2. For completeness, we plot these cohorts in the figure but do not include them in subsequent regression samples. Moreover, they had reached the male retirement age of 60 before the program's abolition in 1993, thus these older cohorts separated by 1993 even in the control region. The intermediate cohorts, born between 1933 and 1943, were exposed to the reform in REBP regions. Exposure to extended benefits was maximal for the cohort born in 1938, who turned 50 at the onset of the reform in 1988 and was then exposed to the reform until it was abolished in 1993, when the 1938 cohort turned 55.

In Panels (a) and (b) of Figure 2, we plot raw data of the fraction of workers in a cohortregion group that separates from their 1988 employer (defined as a worker-establishment match) by 1993q3, the first quarter after the REBP had been abolished. We assign workers by the location of their 1988q2 establishment and leave out workers in the 6 regions eligible for the REBP only through 1991 (TR1 regions in Figure 1 Panel (b)). The red dashed and blue solid lines of Figure 2 Panel (a) show the share of workers in respectively the REBP and control regions who had separated from their 1988 employer by 1993; Panel (b) shows the difference between the share of separators in the REBP and in the control regions. Throughout, there is a clear cohort gradient in the control regions, indicating that older workers' 1988-93 separation share is higher than younger 
workers' share starting with the 1943 cohort and older (who are 50 in 1993), but flat for younger cohorts (born after 1943).

We start our analysis of REBP on separations from the right, with the ineligible young control cohorts born after 1943. These exhibit a separation rate of roughly 40 percent in both regions, and differences between REBP and control regions are flat (in fact close to zero). These parallel trends among younger cohorts validate the identification assumptions of our difference-in-differences design by suggesting that labor market conditions were comparable for the separation margin across REBP and control regions during the study period 18

For affected cohorts born between 1933 and 1943, separations are markedly higher in REBP regions (but not in the non-REBP regions), representing the treatment effect of REBP. At its peak, the difference in the share is about 20 percentage points relative to a control region share of about 50 percent. By comparing slightly older and younger cohorts within the same region, we net out any differences between regions that are constant across cohorts (including market-level effects of the program).

We conclude with the oldest cohorts on the left. A treatment effect emerges even in this cohort: the gradient in the REBP region reveals a higher separation rate than in the non-REBP region. However, note that these cohorts born before 1933 had mostly retired by the end of the REBP, and therefore hardly any workers in these birth cohorts were still employed with their 1988 employer by 1993 in either region.

Potential remaining confounders are shocks or unobservables in separation behavior that vary at the region-by-age level. For instance, pathways to retirement could differ between regions as a consequence of different industry structures. To address this concern, we switch to separation during a fixed age window, 50 to 55, rather than between time periods (years 1988 to 1993) 19 Panels (c) (levels) and (d) (differences) in Figure 2 show that separations between the ages of 50 and 55 increased markedly in cohorts exposed to the REBP relative to older and younger non-exposed cohorts, again compared to the gradients in the control region. The job separation probability increases steadily from around 40 percent in older cohorts to about 50 percent in younger cohorts in the control region (blue solid line, Figure 2 Panel (c)). While the pattern for the pre-1933 and post-1943 cohorts is similar in the REBP regions (red dashed line) with slightly lower shares throughout, separations rise sharply for the treated, intermediate cohorts born between 1933 and 1943. As Figure 2 Panel (d) shows, the magnitude of the increase at peak is almost 20 percentage points.

Finally, Figure 3 plots quarters nonemployed (Panels (a) and (b)) and unemployed (benefit receipt) (Panels (c) and (d)) between 1988q2 and 1993q3, mirroring Figure 2 For the non-exposed younger cohorts, trends are flat and the levels between the regions are essentially the same. For the cohorts exposed to the reform, Figure 3 reveals an economically significant increase of

\footnotetext{
${ }^{18} \mathrm{We}$ also include a figure studying a longer horizon of younger cohorts where trends remain completely flat and close to zero (see Appendix Figure A.3.

${ }^{19}$ In practice, we select the job in the quarter before the 50th birthday (right before aging into REBP eligibility), and the separation outcome in quarter before turning 55 (as the disability and early retirement incentives changed at 55).
} 
nonemployment and a similar increase in unemployment (unemployment insurance/assistance spells) of about three and two quarters at peak, respectively.20 We find similar results when we consider the sample of employed workers at age 50 and track these non-/unemployment outcomes through age 55 (see Appendix Figure A.4).

Regression Estimates of Treatment Effect We complement the graphical evidence with regression estimates on the average treatment effect in Table 2, in a difference-in-differences specification on the population of workers holding a job in 1988 before the onset of the reform, for various outcomes $D_{r c i}$ described below, for worker $i$ in region $r$ in birth cohort $c$ :

$$
D_{r c i}=\beta+\text { REBP Region }_{r}+\text { Treated Cohort }_{c}+v \cdot \underbrace{\text { REBP Region }}_{Z_{r c}} \times \text { Treated Cohort }_{c}+\chi_{r c i} .
$$

The coefficient of interest $v$ captures the effect of REBP eligibility $Z_{r c}$, defined by birth cohort and location. We set $Z_{r c}=1$ for workers located in the REBP region who were born before August 1943, so that they were older than 50 at some point during the active period of the REBP (and zero otherwise, for our control groups) ${ }^{21}$ Here and in subsequent regression analyses, we exclude workers born before August 1933 from our sample because an overwhelming majority had retired by August 1993 anyway. The model includes a baseline REBP region effect and a baseline effect for being a member of a treated cohort. Our regression specification thus exploits within-region, within-cohort variation. ${ }^{22}$ In the main specifications, we cluster standard errors at the level of administrative regions (groups of districts, Arbeitsamtsbezirke) but have also assessed robustness for clustering at other levels. Our main table reports on the cohort-based design (199893 outcomes) in Table 2, we additionally report the age-based estimates (50-55) in Table A.3. finding similar results. We keep the young control cohorts to a five-year range to isolate the workers most comparable to the older, treated cohorts in the labor market. As in the raw data plots, we ignore workers in the few municipalities where the REBP was abolished early.

Table 2 column (1) shows our main result regarding the separations effect of the outside option increase: a 10.9ppt increase in separations by 1993q3 among initially employed workers from their

\footnotetext{
${ }^{20}$ For the more continuous employment measures we also find treatment effects among older cohorts since they were affected by the REBP before reaching retirement age (even though they are overwhelmingly retired by 1993).

${ }^{21}$ That is, we do not condition on the REBP experience requirement (more than 15 years of experience in the last 25 years before unemployment entry). We do so because the correct point in time to measure eligibility is hard to define and reaching the experience requirement during REBP is potentially affected by the policy itself (and workers not meeting the requirement in 1988 may meet it later on). This differs from the design in Lalive et al. (2015), who focus on unemployed workers for whom the experience requirement is well-defined at unemployment entry. More than $90 \%$ of workers older than 50 in our sample fulfill the experience requirement. Accordingly, unreported checks confirmed that the exclusion of workers ineligible due to the experience requirement would not substantially affect our results (neither the treatment effects estimated here, nor the post-REBP predictions and resilience effects studied later). Regarding the location in the REBP region, we draw on the location of workers' establishment and, if that is missing, the residence based on data from AMS, the Austrian employment agency.

${ }^{22}$ Since the project varied over time, we could additionally use variation in the time dimension, e.g., in a triplediff specification with a placebo reform in 1978. Due to the long program duration (five years), we shy away from additionally using variation over time for identification since we would have to rely on long lags to implement a placebo reform analysis (including the post-REBP analyses) that would not be contaminated by the REBP.
} 
1988q2 employer. This represents about a 27\% increase in separations, relative to a counterfactual separation rate of $40.5 \%$ in the absence of REBP (regression constant plus the baseline effects for treatment region and old cohorts). The $95 \%$ confidence interval for the separation effect ranges from 2.9 to $18.9 \mathrm{ppt}$.

We then split the effects on separations into the two possible types: separations either into nonemployment (i.e. those without employment at another employer between 1988 and 1993) and the complement (i.e. separations of individuals who had employment at another employer at some point between 1988 and 1993). REBP-induced separations are entirely made up of the former (separations into nonemployment). In column (2), we report a sizable increase in nonemployment separations of 12.0ppt (SE 4.3ppt). Column (3) of Table 2 reports effects on quarters nonemployed between 1988 and 1993, finding a positive effect of 1.46 quarters (SE 0.38). Column (4) reports that quarters unemployed (defined as unemployment insurance/assistance receipt) increased by 0.95 quarters (SE 0.53). Column (5) shows that a large share of the increase in nonemployment can be accounted for by a reduction of 1.05 quarters in continuous employment with the initial employer (SE 0.37), such that our setting does not represent the standard temporary layoffs mechanism (Feldstein, 1976).

Taken together, the evidence shows that REBP benefit extensions caused job separations (10.9ppt or $27 \%$ increase), shorter employment with the initial employer, and a tantamount increase in unemployment ${ }^{23}$ This effect comes in response to a treatment of 209 weeks of potential benefit duration compared to 52 weeks in the older control group, i.e. a differential four-fold increase in potential duration of UI benefits by three years.24

\subsection{Complier Characterization of REBP Separators}

We next dissect the characteristics of the incremental REBP separators - marginal jobs. They are compliers: they would have survived in the absence of the treatment (hence still present in the control group) but did not survive when exposed to it (treatment group). To study their attributes, we extend the complier analysis method (Imbens and Rubin, 1997, Abadie, 2003) to difference-in-difference settings such as ours. We compare the marginal matches separating due to REBP ("compliers") to those matches that separate even in the control group i.e. absent REBP ("always-separators") and the matches that survive even in the treatment group i.e. despite REBP ("never-separators"). All characteristics we consider are measured before the start of REBP in 1988 and thus are unaffected by the reform by construction.

We will conclude that studying the attributes of these REBP separators is neither exactly nor definitely informative about their efficiency properties, because worker, firm and joint job surpluses

\footnotetext{
${ }^{23}$ We also replicate the separation effects based on survey data from the Austrian Micro Census. The survey also allows us to measure ostensible types of separations, e.g., quits, based on a retrospective classification by survey respondents (see Appendix Section B).

${ }^{24}$ Both groups had at most 30 weeks PBD in 1988, but a national reform in 1989 increased that level to 52 weeks, leading us to choose this benchmark given the program duration through 1993.
} 
are not directly observed - the key motivation for our study to focus on a revealed-preference test of the Coasean framework based on the introduction and then abolition of a surplus-relevant nonemployment subsidy program, which we continue in Section 4 .

\subsubsection{Methodology: Complier Analysis in Difference-in-Differences Settings}

In Appendix C.1, we provide a detailed, stand-alone methodological guide and proof to extend complier analysis (Abadie, 2003) to difference-in-differences contexts, building on an additive separability assumption for attributes. We provide intuition and then a summary of the formal methodology below.

Intuition of Complier Analysis For the ease of illustration, suppose that policy $Z$ is randomly allocated and leads to additional separations of, by definition, marginal matches $M$. We would like to distill information about the attributes carried by these marginal jobs $M$ (for example the mean $\bar{x}$ of some scalar variable $x$, but the methodology can be applied to identify any moment of the distribution). Empirically, we observe the attributes as well as the share of treatment-group separators and control-group separators. On their own, treatment and control separators stem from largely inframarginal surplus ranges, of surplus at least or at most 0 . Yet, we can back out the attributes of marginal matches (those with close to 0 surplus and hence swept up by REBP) by rearranging the expression for treatment separators, which are the union of control separators and marginal matches, such that $\bar{x}^{Z=1}$ is just the average of $\bar{x}^{Z=0}$ and $\bar{x}^{M}$, weighted by their share in total separations:

$$
\begin{aligned}
\bar{x}^{\mathrm{Z}=1} & =\frac{\delta^{0}}{\delta^{1}} \times \bar{x}^{\mathrm{Z}=0}+\frac{\delta^{1}-\delta^{0}}{\delta^{1}} \times \bar{x}^{M} \\
\Leftrightarrow \bar{x}^{M} & =\frac{\delta^{1}}{\delta^{1}-\delta^{0}} \times \bar{x}^{\mathrm{Z}=1}-\frac{\delta^{0}}{\delta^{1}-\delta^{0}} \times \bar{x}^{\mathrm{Z}=0} .
\end{aligned}
$$

The weights are measured as group-level separation rates, along with treatment effect $\delta^{1}-\delta^{0}$. Below we extend complier analysis to difference-in-differences settings such as ours; to our knowledge, the formal derivation is new.

Setup We set up a potential outcomes framework for the difference-in-differences model (1) estimating the effect of REBP on separations among workers holding a job already pre-REBP in 1988, with fixed effects for region $r$ and cohort $c . D \in\{0,1\}$ indicates whether a given worker separates from their initial, pre-reform job by the end of the reform period (whether she was treated or not). $Z \in\{0,1\}$ captures whether workers are REBP-eligible (1) or not (0). $D_{0}$ and $D_{1}$ denote the potential values of $D$ for $Z=0$ and $Z=1\left(D=Z D_{1}+(1-Z) D_{0}\right)$. REBP varied by region $r \in\left\{r_{0}, r_{1}\right\}$ and cohort $c \in\left\{c_{0}, c_{1}\right\} . r_{0}$ is the control region and $r_{1}$ is the REBP region. $c_{0}\left(c_{1}\right)$ denotes ineligible (eligible) cohorts. We thus have that $Z=1$ for $\left(r_{1}, c_{1}\right)$ only, and $Z=0$ for other combinations $\left(r_{1}, c_{0}\right),\left(r_{0}, c_{0}\right)$ and $\left(r_{0}, c_{1}\right)$. Always-Separators have potential outcomes $\left(D_{0}=1, D_{1}=1\right)$ and share 
$\pi^{A S}$. Never-Separators have potential outcomes $\left(D_{0}=0, D_{1}=0\right)$ and share $\pi^{N S}$. Compliers-marginal jobs - have potential outcomes $\left(D_{0}=0, D_{1}=1\right)$, i.e. they separate when affected by the policy but would otherwise not separate, and share $\pi^{C}$. Figure 4 Panel (a) illustrates the empirical shares of these three groups from identified by our difference-in-difference design.

Identification and Estimation of Complier Means We show how complier characteristics in difference-in-differences designs are identified under familiar first stage, independence, and monotonicity assumptions as well as a new additive separability assumption for characteristics $x$. The latter is analogous to assumptions of parallel trends or additive separability in recent work extending the instrumental variables setup to difference-in-differences settings (De Chaisemartin and D'Haultfoeuille, 2018; Hudson et al., 2017).

Under the four assumptions above, we can express the characteristics of compliers, $\mathbb{E}\left[x \mid r_{1}, c_{1}, D_{1}=\right.$ $\left.1, D_{0}=0\right]$, in terms of estimable quantities:

$$
\mathbb{E}\left[x \mid r_{1}, c_{1}, D_{1}=1, D_{0}=0\right]=\frac{\pi_{r c}^{C}+\pi_{r c}^{A}}{\pi_{r c}^{C}} \cdot \mathbb{E}\left[x \mid r_{1}, c_{1}, D_{1}=1\right]-\frac{\pi_{r c}^{A}}{\pi_{r c}^{C}} \mathbb{E}\left[x \mid r_{1}, c_{1}, D_{0}=1\right] .
$$

Under the independence and additive separability assumption, the quantities on the right-hand side can all be measured in the sample so that complier characteristics are identified in DiD IV settings such as ours (see Appendix C).

In Appendix C.1. we also present a one-step regression estimation of complier attributes in difference-in-differences settings. For inference, we use the non-parametric bootstrap to arrive at a sampling distribution of (4).

\subsubsection{Results}

Figure 4 and Appendix Table A.2 provide an overview of means for compliers, never-separators, and always-separators, based on pre-reform data from 1988, along with p-values for mean differences between compliers and the other groups.25 As before, we focus on cohorts born after 1933. Figure 4 Panel (b) plots differences between compliers and never-separators, normalized by the standard deviation for each variable. In Figure 4 Panel (c) (and the last column of Appendix Table A.2, we also present estimates of treatment effect heterogeneity of REBP on separations by estimating (1), interacting treatment with covariate indicators (we split continuous variables at the median), largely mirroring the results of the complier analysis ${ }^{26}$

${ }^{25}$ We provide a test of an implication of the additive separability assumption and report results in Appendix Table A.1. While we find statistically significant differences for some variables we consider, the magnitudes of the respective differences are small.

${ }^{26}$ Specifically, the density of nearly marginal matches carrying $x$, backed out from treatment and control separators, is: $s^{M}(x)=\frac{\delta^{Z=1}}{\delta^{Z=1}-\delta^{Z=0}} \times s(x)^{Z=1}-\frac{\delta^{Z=0}}{\delta^{Z=1}-\delta^{Z=0}} \times s(x)^{Z=0}$. Alternatively, $s(x)$ is the product of the relative treatment effect on separations among type- $x$ jobs compared to the average effect, times the share of type- $x$ job in the initial sample, $f(x)$ : $s^{M}(x)=\frac{\delta^{Z=1}(x)-\delta^{Z=0}(x)}{\int_{x}\left[\delta^{Z=1}(x)-\delta^{Z=0}(x)\right] d F(x)} \cdot f(x)$. See also results in Angrist and Pischke 2008) and Angrist and Fernandez-Val (2013) for Bernoulli-distributed covariates. 
We briefly summarize the results here. At a broad level, observable characteristics of separators provide some (though limited and mixed) evidence consistent with low and declining surplus. Another limitation is that each given attribute may carry multiple potential interpretations. Finally and most importantly, and a priori, observable complier attributes are merely potentially surplus-relevant, in that they are mere correlates of those underlying surplus-relevant factors in V. Appendix C.3 provides a full discussion of the results.

We find that, compared to jobs surviving the treatment, compliers are primarily blue-collar workers in declining establishments from manual labor-intensive industries with higher shares of disability or sickness among older workers. Compared to surviving jobs, marginal jobs had a higher risk of long nonemployment duration and were more prevalent in shrinking industries and firms.

But the analysis also reveals that the REBP separators stemmed from many pockets of the Austrian labor market and would therefore not be definitely identified based on their pre-separation attributes. For example, we remarkably find no evidence for wage differences between compliers and never-separators - perhaps consistent with wage compression or wage rigidity.

This difficulty to trace surplus concepts on the basis of observables motivates our revealedpreference test, to which we devote the rest of the paper, tracking jobs after the surprise abolition of REBP in 1993.

\section{Puzzle to the Coasean Benchmark: No Attenuated Separations Among REBP Survivors After its Surprise Abolition in 1993}

We now exploit the surprise abolition of the reform in August 1993 (described in Section 2) to study whether REBP "survivors" - jobs that existed before the onset of the reform in 1988 and continued through its abolition in 1993 - subsequently exhibited lower separation rates unconditionally and in response to negative labor demand shifts. That is, we essentially test for a dynamic known as "harvesting effects" in demography (see, e.g., Schwartz, 2000; Basu, 2009), usually applied to transitory negative health shocks (e.g., heat waves) that induce deaths among lowhealth individuals and reduce the subsequent average mortality rate of the survivors.

We find that, after the abolition of the reform, the "survivors" in the dramatically shrunk former treatment group exhibited exactly the same separation behavior as the control group - on average, and in response to industry and firm labor demand shifts. Together, the large quantity of REBPinduced separations before 1993, and the zero differential post-REBP behavior, are inconsistent with the core prediction of resilience from our preferred Coasean benchmark, for which we lay out intuitions in Section 4.1 and in formal detail in Section 5 . We will dedicate the rest of the paper to understanding this result. 


\subsection{Informal Discussion of Coasean Prediction: Resilience from Missing Mass of Marginal Matches}

To motivate our study of post-abolition separation outcomes, we now summarize the intuitions underlying the predictions from the Coasean framework, which we fully present in a formal model as well as in a structural estimation in Section 5 .

The Coasean benchmark assumes that efficient bargaining reallocates surplus between the worker and the firm so that any job with positive joint surplus will be viable, and a match will be destroyed when joint surplus turns negative. This framework implies an ordered set of jobs that separate in response to a surplus-relevant shock exceeding the initial surplus level: small shocks destroy jobs with small joint surplus, larger shocks will additionally sweep up jobs with larger joint surplus. Hence, REBP - which reduced surplus by boosting worker's nonemployment outside option - ought to have extracted jobs with surplus below the REBP surplus shift, and thereby changed the composition of surviving employment relationships towards jobs with higher initial surplus, or gross-of-REBP surplus. Crucially, we exploit the abolition of REBP, which reduces again the outside options, aligns surplus between treatment and control groups, and restores the allocative surplus distribution of the surviving jobs to the right. By then focusing on job survivors (active at the onset of REBP and through its abolition date) in the panel, the former treatment group then features a missing mass of marginal matches destroyed by REBP, which are still active in the control group. A testable prediction of the Coasean view of jobs is that right after the REBP abolition, the formerly treated group of REBP survivors should be more resilient in their separation rate compared to the control group, where these marginal, low-surplus jobs are still present. As time progresses after the abolition, reshuffling of idiosyncratic surplus may weaken this prediction by somewhat "filling" the hole again, as we discuss in the formal model.

Figure 5 illustrates this logic. Panel (a) represents a hypothetical distribution of joint job surplus right before REBP takes place. During REBP, all jobs with negative joint surplus (i.e. all jobs in the left-most, light blue area) separate both in the control and in the treated regions. In the treated regions, there is an extra set of jobs (the polka-dotted region) that separate only because exposed to REBP but would survive active absent REBP. We estimated this treatment effect of REBP on separations as a 10.9ppt (about 27\%) increase from a separation rate of 40.5ppt in the absence of the program (Table 2, column (1)).

These marginal matches will be missing in the treatment group once the reform is abolished. This missing mass is illustrated in Panels (b) and (c), which plot the distribution of surviving jobs by the end of REBP, in the treated and in the control regions respectively. This missing mass is large in the data: by the end of REBP, the eligible older cohorts in the REBP region has shed 10.9ppt of workers from their ranks. Among older cohorts in the control group, who had a $40.5 \%$ separation rate, these marginal, low-surplus jobs still comprise $\frac{0.109}{1.0-0.405}=18 \%$ of active jobs.

The crucial feature of our two-step research design becomes clear when contrasted to an alternative naive design studying differential separation behavior during REBP, without the sharp program abolition. In such a design, the treatment group is indeed subject to a stricter cutoff, 
but only in terms of gross-of-REBP surplus. Yet, net surplus is allocative during REBP. The separation rates as well as responsiveness depend only on the local density of surplus around the two thresholds, which could be larger or smaller following REBP and would simply inherit the properties of the surplus distribution, which is not measured ${ }^{27}$ So, on their own, these outcomes during REBP are uninformative about the efficiency properties of these initial separations.

Given the tension between data and Coasean predictions, in Section 6 we will discuss how nonCoasean settings - which do not feature a one-dimensional ordering of jobs by joint surplus - can rationalize our findings. For example, suppose that wages were rigid or layoff/firing restrictions were active, so that REBP selected workers into separation based solely on worker surplus (as it boosted workers' outside options) rather than joint job surplus. Then, resilience would emerge to worker surplus shifts, but not necessarily to firm surplus shifts.

\subsection{Baseline Results}

We now present our study of the post-REBP separation behavior as a revealed-preference test of the prediction of the baseline Coasean model, that the extraction of marginal jobs leads to more resilience in the former treatment group, which lacks marginal matches, compared to the former group control group, where the marginal matches are still present.

Plotting Raw Data: Post-REBP Separation Rate Gradients by Cohort In Figures 6 (levels) and 7)(differences between regions) we plot the post-REBP separation cohort gradient among "REBP survivors" in the former treatment and control groups: among the jobs already active right before the onset of REBP in 1988 that continued through its abolition in 1993. In practice, we allow for REBP spillovers due to layoff notices and explicit grandfathering that the law permitted for pre-scheduled layoffs (see Section 2) so we focus on jobs that survived through the first quarter of 1994 for the post-REBP analysis. ${ }^{28}$ Hence, our cutoff defining the survivor sample is 1994q1. Except for this sample restriction, the figures mirror the cohort gradients of separations in Figures 2 and 3 . We explore the fraction of REBP survivors subsequently separating at various horizons.

Figures 6 and 7 reveal that, comparing a given treated cohort and its peer control cohort, there are no post-REBP separation differences whatsoever among surviving jobs previously exposed to the REBP compared to those those that were not. The raw data on separation-cohort gradients provide nonparametric evidence for the absence of resilience.

As a quantitative benchmark, both figures plot the predicted separation rate based on the Coasean model, which we formally derive and discuss in Section 5 Intuitively, the Coasean model derived in Section 5 predicts smaller separation effects in the formerly treated group post-abolition as initial

\footnotetext{
${ }^{27}$ The specific statistical property guiding separations in response to small shocks would locally be the reverse hazard rate, discussed in detail for extensive margin employment adjustment more generally in Mui and Schoefer (2019) with a focus on spot-market labor supply.

${ }^{28}$ This grandfathering likely played a role in the additional increased separation rate in the REBP region immediately after the program's abolition. Appendix Figure A.5 documents these additional separations by separating the withincohort regional difference from 1993q3 to 1994q1 into quintiles of industry growth over the same time period. In Section 6.4. we clarify that a version of the non-Coasean model can rationalize these patterns even without grandfathering.
} 
treatment effects of REBP on separations in a given cohort become larger (depicted in Figure 2 Panel (b)). This is because these cohorts feature a larger mass of margin matches at the point of REBP abolition, and separations in these formerly treated cohorts pick up only once the counterfactual proxied for by the peer cohort in the control region - exceeds the initial treatment effect. For now, we note that the gap between the prediction and the Coasean benchmark is quantitatively large, confirming that our test has power.

Regression Evidence To gauge quantitative effects and to assess confidence intervals, we again estimate the difference-in-differences model in Equation (1) on the current sample (REBP stayers) and the post-REBP separation outcomes. We choose 1994-1996 as one example but report the full set of horizons in Appendix Tables A.4 through A.6. Specifically, we track the labor market status of "REBP survivors" from 1994q1 through 1996q1, following the same birth cohorts and regions but with the additional "survival" restriction of being observed in the same establishment in 1988q2 and 1994q1. Just as in the previous section, we ignore those working in the regions where REBP was abolished by the end of 1991 . We estimate, for a series of outcomes, the model:

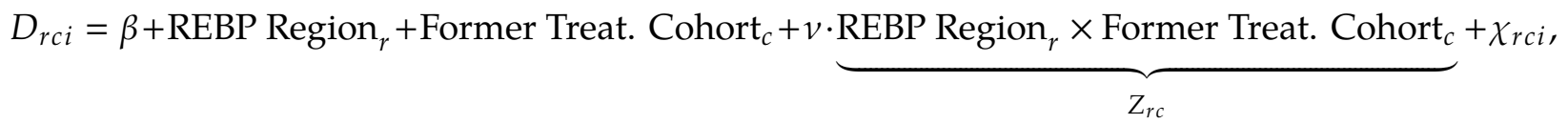

among the sample of survivors in both groups for both regions. Again, we focus on a sample of workers born after 1933 and include younger control cohorts born from 1943 to 1948 never eligible for the REBP. As before, we cluster standard errors at the administrative region level. The coefficient of interest remains $v$, the difference-in-differences between REBP-eligible and -ineligible cohorts and regions.

We report results in Table 3 This basic difference-in-differences analysis of post-abolition separation behavior shows that the REBP survivors were not more resilient in the post-abolition phase. If anything, we find point estimates indicating small, positive effects - the opposite of resilience. In column (1), studying separations by 1996 as outcome, we report an estimate of $v$ of 0.6ppt (SE 0.9). The tight confidence intervals allow us to rule out effects smaller than $-1.2 p p t{ }^{29}$ We find similar effects on the separation-into-nonemployment margin in column (2). In columns (3) through (5), we consider more continuous measures of time in nonemployment, time on unemployment benefits or assistance, and continuous employment with the original employer. Across all margins, we detect no resilience and the point estimates are small with tight confidence intervals.

${ }^{29}$ At longer horizons, we find small positive effects and can statistically rule out any negative effects (see Appendix Tables A.4 through A.6. We also report an alternative version of Table 3 where we drop workers close to the retirement age (see Appendix Table A.7). In that sample we again find small positive and statistically significant separation effects, i.e. the opposite of more resilience. 


\subsection{Labor Demand Shocks}

We present additional results below to illustrate the robustness of the absence of differential postREBP separation behavior. This is a subset of the long series of unreported robustness checks in search for resilience we have conducted 30 Here, we test whether proxies for negative labor demand shocks may unmask the potentially underlying missing mass of marginal matches that, by the Coasean view, should render the formerly treated group less sensitive to shocks. Both at the industry and at the establishment level, we find that separation behaviors of the groups remain indistinguishable.

Heterogeneity by Industry Growth Since we study the separation margin, the Coasean benchmark would predict resilience to negative shocks in particular. We therefore plot the differential separation rates among jobs that survived REBP separately for the top, middle and bottom tercile of the industry growth distribution from 1994 onward (to the reference year in which we define separations after REBP) in Figure 8 Even in declining industries (the 1st tercile has negative employment growth), in which joint surplus is arguably shrinking, REBP cohorts do not exhibit relative resilience compared to the control group.

Establishment-Level "Hockey Sticks" We construct proxies for separation-inducing establishment labor demand shocks by tracing out "hockey stick" graphs (Davis et al., 2013): separation rates are steeply negative in the negative net employment growth region (largely driven by layoffs), then feature a kink around zero employment growth and are somewhat positive (due to turnover associated with hiring). Exploiting the matched employer-employee dimension of our population data, we replicate these graphs in Figure9 9 Panel (a), where we plot annual separation rates for all male employees employed in Q1, by bins of annual net employment growth (where both employment and separations are hence defined beyond our worker sample).

Figure9 Panel (b) plots cohort-region-specific the separation rates through 1996 (we include other end points 1995, 1997, 1998 in Appendix Figure A.6), among the sample of jobs that survived the REBP period through 1994q1. For this analysis, we focus on cohorts from 1936 through 1948 to avoid retiring cohorts (in Appendix Figure A.6, we focus on cohorts born after 1935, 1937, and 1938, respectively). The results are naturally noisier due to the shrinking sample, but are still informative because the Coasean view would predict that separations occur by a pecking order following the ranking of job surplus within an establishment. We estimate linear slopes separately for shrinking and growing establishments and for four separate groups: by birth cohort eligibility (July 1936 to July 1943 vs. August 1943 to July 1948) and regional eligibility.

The slopes for the old ineligible, non-REBP-region workers and the formerly treated and formerly eligible (old REBP-region) workers lie almost on top of each other. This similar separation behavior provides additional evidence that the massive extraction of potentially marginal jobs due

\footnotetext{
${ }^{30}$ In fact, the original motivation of this paper was to establish such resilience, and our firm prior was to find such resilience.
} 
to REBP does not seem to affect subsequent layoffs (or separators) at firms.

Lastly, we report cohort-specific slopes, estimating for each birth-year cohort $c$ and region $r$ with establishment employment growth $\hat{n}$,

$$
\begin{aligned}
D_{i, 1994+t}= & \beta_{1}^{c, r} \cdot\left(\hat{n}_{e(i), 1994+t} \times \mathbb{1}\left(\hat{n}_{e(i), 1994+t}<0\right) \times \mathbb{1}_{c(i), r(i)}\right)+\beta_{2}^{c, r} \cdot\left(\hat{n}_{e(i), 1994+t} \times \mathbb{1}_{c(i), r(i)}\right) \\
& +\beta_{3}^{c, r} \cdot\left(\mathbb{1}\left(\hat{n}_{e(i), 1994+t}<0\right) \times \mathbb{1}_{c, r}\right)+\beta_{4}^{c, r} \cdot \mathbb{1}_{c(i), r(i)}+\xi_{i}
\end{aligned}
$$

where $D_{i, 1994+t}$ is an indicator of whether a worker $i$ employed in 1994q1 is still employed with the same establishment $e$ in Q1 of year $1994+t$. Our worker sample is again the 1988-94 job stayers.

$\hat{n}_{e(i), 1994+t}$ is the growth in total establishment employment between 1994q1 and Q1 of year $1994+t$, in the establishment $e$ where individual $i$ is employed in 1994. $\mathbb{1}_{c, r}$ is an indicator for being in cohort $c$ and region $r$. The coefficient of interest is $\beta_{1}^{c, r}+\beta_{2}^{c, r}$, the sensitivity of separations to downsizing at the establishment level by year of birth $c$ and region $r$.

Figure 9 Panel (c) plots the estimates from this regression for separations/growth rates from 1994 to 1996 (i.e. $t=2$ ), with similar results for other years (reported in Appendix Figure A.6). In both the REBP and the non-REBP region, the 1988-94 job stayers (REBP survivors) exhibit a downward-sloping sensitivity gradient in birth date, indicating that older workers appear shielded from a given establishment shrinkage event, perhaps due to seniority rules (see Section 2), larger job values for human capital reasons (Oi, 1962), or lower outside options among these older workers. Yet, the lines lie on top of each other: REBP and non-REBP birth cohorts exhibit the same sensitivity of separations to negative establishment labor demand shifts. (If anything fact, the REBP cohort appears slightly more exposed to firm shocks.)

\section{Can the Coasean Model Rationalize the Absence of Resilience?}

We now clarify under which conditions the Coasean view of jobs can rationalize our findings of no resilience post-abolition. Section 5.1 sets up the general model; Sections 5.2 and 5.3 view the empirical design through the lens of that model. We start by formally showing that in the Coasean setting, the formerly-treated group should be less sensitive to any subsequent negative shocks, as long as there is some persistence in job-level idiosyncratic surplus after the abolition, say from 1994 to 1995 . We then show that only under narrow conditions can the Coasean setting explain the absence of resilience: namely we would require no persistence whatsoever, such that from 1994 to 1995, the first year after the abolition, the surplus distribution of the formerly treated group would need to converge immediately and fully to that of the control group. In Section 5.4, we structurally estimate which weight the economy puts on two extremes concerning the evolution of idiosyncratic job surplus: perfect persistence vs. full reshuffling of surplus. We find that in order to account for our empirical results, the point estimates reveal that essentially $100 \%$ (lower confidence interval: $89 \%$ ) of labor market cells of our sample must feature no persistence whatsoever in joblevel surplus. Section 5.5 discusses the model features as well as the interpretation of this horse 
race. In Section 6, we then lay out how a non-Coasean setting can rationalize our results of no resilience, even with persistence in idiosyncratic, job-level surplus.

\subsection{Coasean Bargaining and Efficient Job Separations}

The following framework formalizes how improvements in worker outside option affect separations and truncate the distribution of job surplus among the surviving jobs, generating a missing mass of marginal matches, thereby predicting resilience after the abolition of REBP.

Jobs and Surplus Generally, jobs carry worker surplus $S^{W}$ and firm surplus $S^{F}$, each of which must be non-negative: each party $i \in\{W, F\}^{\prime}$ s inside job value $V_{\text {In }}^{i}$ (amenities, productivity,...) plus/minus wage $w$ (with which the parties can transfer utility in terms of e.g. present values), must amount to at least her (separation) outside value $V_{\text {Out }}^{i}$ (unemployment, retirement, working for another firm, the value of a vacancy and hiring another worker,...):

$$
\begin{aligned}
S^{W}\left(w, \mathbf{V}^{W}\right) & =V_{\text {In }}^{W}+w-V_{\text {Out }}^{W} \geq 0, \\
S^{F}\left(w, \mathbf{V}^{F}\right) & =V_{\text {In }}^{F}-w-V_{\text {Out }}^{F} \geq 0,
\end{aligned}
$$

where $\mathbf{V}=\left\{V_{a}^{i}\right\}_{i \in\{W, F\} ; a \in\{\text { In,Out }\}}$, and sometimes $\mathbf{V}^{i}=\left\{V_{a}^{i}\right\}_{a \in\{\text { In,Out }\}}$. Additionally, we define joint job surplus, which the wage splits between the worker and firm:

$$
S(\mathbf{V})=\overbrace{V_{\text {In }}^{W}+V_{\text {In }}^{F}-V_{\text {Out }}^{W}-V_{\text {Out }}^{F}}^{S^{W}\left(w, \mathbf{V}^{W}\right)+S^{F}\left(w, \mathbf{V}^{F}\right)} .
$$

Figure 10 Panel (a) plots the two-dimensional job space. The x-axis denotes worker surplus, and the $y$-axis denotes firm surplus. The figure plots various case studies of jobs characterized by different surplus coordinates. The solid circles $(\bullet)$ denote gross-of-wage surpluses, i.e. $V_{\text {In }}^{W}-V_{\text {Out }}^{W}$ for the worker and $V_{\text {In }}^{F}-V_{\text {Out }}^{F}$ for the firm. This is the surplus combination these job "fundamentals" would trigger before wage setting, or equivalently in the scenario of a zero wage. The empty circles (o) denote net of wage surpluses: for each gross job, we provide various examples of potential wages. Wages move net surpluses of the parties along the 135-degree i.e. iso-joint-surplus line.

The figure also partitions jobs into four regions of viability: feasible jobs (north east, solid line), quits (north west, dashed line), layoffs (south east, dotted line) and mutual separations (south west, dot-dash-patterned line). For a job to be viable, it must be in the north east corner, providing positive surplus to both parties. Three natures of separations are represented by the three remaining corners. Quits emerge if the worker is in negative surplus territory, while the firm would prefer to continue. This case would emerge in job $A$, which is "born" in such a potential quit case. Yet, thanks to redistribution in form of a positive wage, the job can be moved into the viable quadrant, $A_{1}$. The wage can also "overshoot": job $A_{2}$ has too high a wage, playing the job firmly into positive territory from the worker's perspective, but pushing it into negative firm 
surplus territory, leading to a layoff, along with all jobs in the south east quadrant. By contrast, job $B$ is born in the feasible region even with a zero wage, as one would imagine with, e.g., an internship or a high-amenity job, for which workers would work for free. Yet the figure plots two ways to have wages entail separations: $B_{1}$ has too positive a wage, leading to a layoff. $B_{2}$ has a too negative a wage, entailing a quit. Job $C$ goes one step further, where a no-wage scenario would have the firm prefer a layoff, but too low (negative) a wage would entail a quit $\left(C_{1}\right)$, whereas any positive wage would leave the job in layoff territory $\left(C_{2}\right)$. By contrast, doomed jobs such as $X$ are born in negative surplus territory for both parties, and so provide negative joint surplus such that no wage can be found to fulfill both participation constraints. Wages in the south west quadrant hence entail a mutual separation (otherwise a quit or layoff). Finally, $M$ is a "marginal" job, carrying exactly zero joint surplus. That job is born in, e.g., quit territory, but can be rendered feasible with a unique wage that moves that job into the origin, where both parties enjoy exactly zero unilateral surplus. Any increase [decrease] from that wage will entail a layoff [quit].

Coasean Bargaining The essence of the Coasean setup is that the parties find a wage within the bargaining set of reservation wages $w \in\left[\underline{w}^{W}, \bar{w}^{F}\right]$, where $S^{W}\left(\underline{w}^{W}, \mathbf{V}^{W}\right)=0$ and $S^{F}\left(\bar{w}^{F}, \mathbf{V}^{F}\right)=0$, any of which implements the bilaterally efficient allocation: forming and maintaining matches that carry non-negative joint - rather than private - job surplus (i.e. whenever $\bar{w}^{F} \geq \underline{w}^{W}$ ) 31 As a result, the two-dimensional surpluses that determine job viability, Equations (7) and (8), collapse to a one-dimensional, single allocative concept of joint surplus (9) defining job viability and hence separations (defined next in Equation (10)), which the parties split to leave both participation constraints fulfilled.

In Figure 10 Panel (a), Coasean i.e. efficient bargaining, by flexibly moving jobs along the iso-joint-surplus curve, renders feasible all jobs born north east of the marginal-jobs frontier (i.e. those with positive joint surplus).

Separations With Coasean bargaining, we observe a separation if the joint surplus moves into negative territory. Suppose job values evolve following a Markov process $k\left(\mathbf{V}^{\prime} \mid \mathbf{V}\right)$, where, going forward, $x^{\prime}$ denotes the next-period value of $x$. Then, for a job of value vector $\mathbf{V}$ the probability of separating next period is the probability of transitioning to job values $\mathbf{V}^{\prime}$ that yield negative joint surplus:

$$
\mathbb{d}(\mathbf{V})=\int_{\mathbf{V}^{\prime}} \mathbb{1}\left(S\left(\mathbf{V}^{\prime}\right)<0\right) k\left(\mathbf{V}^{\prime} \mid \mathbf{V}\right) d \mathbf{V}^{\prime} .
$$

Below, we formally explain why our evidence from the empirical REBP and its abolition is difficult

\footnotetext{
${ }^{31}$ For example, by Nash bargaining, the worker (firm) receives their outside option [or reservation wage], plus fraction $\beta$ (resp. $1-\beta$ ), the party's bargaining power, of the surplus (the reservation wage difference):

$$
\max _{w}\left(\left[V_{\mathrm{In}}^{W}+w\right]-V_{\text {Out }}^{W}\right)^{\beta} \cdot\left(\left[V_{\mathrm{In}}^{F}-w\right]-V_{\text {Out }}^{F}\right)^{1-\beta} \Rightarrow w^{N}=\left[V_{\text {Out }}^{W}-V_{\mathrm{In}}^{W}\right]+\beta \cdot S=\underline{w}^{W}+\beta \cdot\left[\bar{w}^{F}-\underline{w}^{W}\right]
$$
}


to reconcile with the Coasean view, unless one is willing to make extreme assumptions on the evolution of idiosyncratic job surplus after REBP captured by the Markov process.

The UI Extension (REBP) We think of the treatment, an increase in UI generosity (a binary variable $Z \in\{0,1\}$ such that $b_{Z}=b_{0}+Z \times \Delta b$, with $Z=1$ for the treatment group and $Z=0$ for the control group), as primarily improving the worker's outside option $V_{\text {Out }}^{W}(b)$, such that the worker surplus size of the shock is $\varepsilon_{b}^{W \prime}=V_{\text {Out }}^{W}\left(b_{0}+\Delta b\right)-V_{\text {Out }}^{W}\left(b_{0}\right)>0$, where our convention is that a positive $\varepsilon_{b}^{W \prime}$ denotes a negative shock to worker surplus (arising from an increase in the worker outside option), and more generally we will use positive $\varepsilon^{i}$ to denote negative surplus shocks going forward - which we assume to be a homogeneous level shifter 32 In our Austrian context, described in Section 2, even worker-sided quits receive full benefits (after a brief waiting period). There is no experience rating. UI take-up is high. Alternatively, due to moral hazard and efficiency-wage mechanisms, the worker's improved outside option may lower productivity (Shapiro and Stiglitz, 1984; Akerlof and Yellen, 1986; Katz, 1986) and thus the firm's inside value, $V_{\text {In }}^{F}(b)$. Or, surplus may fall if implicit layoff/firing costs fall when workers stand to lose less from a separation, in effect increasing $V_{\text {Out }}^{F}(b){ }^{33}$ Still, these alternative mechanisms ultimately arise from the worker's outside option. In Appendix A, we calculate the cash value of the reform as $71 \%$ of a worker's annual salary.

Equilibrium Effects of REBP on Job Values $\quad b$ may also enter the firm's separation value through shifts in recruitment costs or quality of replacement hires. However, our empirical difference-indifferences would net out such mechanisms with the second control group (i.e. we additionally compare slightly younger, ineligible control workers across the REBP and non-REBP regions), such that the treatment is the differential exposure of the treatment group to the program. These within-region ineligible slightly younger control workers are presumably close substitutes to and in the same labor market as their slightly older peers. We therefore suppress such market-level or spillover effects of the REBP shift on control groups or the treatment groups' inside values in our model notation. We discuss this argument in detail in Section 5.5 below.

\subsection{Effects of REBP in the Coasean Setting: Intuitions from Contour Maps}

We now read our full set of empirical findings during REBP and following its abolition through the Coasean lens, starting with intuitions from contour maps, then returning to our formal model in Section 5.3 .

To study the group-level effects of REBP, we now switch gears from individual job case studies to the distribution of heterogeneous jobs. Figure 11 depicts, as our expositional example, the

\footnotetext{
${ }^{32}$ With heterogeneous treatment effects, marginal jobs also comprise workers with particularly large valuation of REBP, a possibility we discuss and address empirically and conceptually in Section 5.5. where we conclude that this possibility is unlikely to revise our main results.

${ }^{33}$ For example, since firms may backload compensation due to agency concerns, firms' flow surplus and the continuation value from older workers is negative gross of layoff/firing costs, generated, e.g., by the erosion of the firm's reputation to honor such implicit contracts (Lazear. 1979, 1981, Hall and Lazear. 1984, Bewley. 2002).
} 
contour maps of the joint distribution of worker and firm surplus embedded in the four quadrants of Figure 10 Panel (a) and illustrates the evolution of jobs: the treatment effect of REBP, which jobs it destroys, and the consequences for post-REBP job dynamics. The upper panels plot the Coasean i.e. efficient bargaining cases. (The lower panels plot the analogous non-Coasean (fixed-wage) case, which we will discuss in Section 6) The figures plot contour maps of the density of the joint distribution of firm (y-axis) and worker (x-axis) surpluses; darker shades indicate higher densities, at the example of a bivariate normal distribution.

We start with the Coasean setting in Panels (a.C), (b.C), and (c.C). Panel (a.C) depicts how REBP lowers joint surplus by $\varepsilon_{b}^{W \prime}=V_{\text {Out }}^{W}(b+\Delta b)-V_{\text {Out }}^{W}(b)>0$. That is, REBP shifts all potential jobs to the west, along worker surplus, by $\varepsilon_{b}^{W \prime}$ and thereby extracts - pushes into separation - all matches with "gross-of- $\varepsilon_{b}^{W \prime \prime \prime}$ surplus below $\varepsilon_{b}^{W \prime}$. These jobs drive the treatment effect on separations documented in Section 3

After REBP is abolished, depicted in Figure 11Panels (b.C) (treatment group) and (c.C) (control group), each surviving formerly treated match's surplus is restored again to its peer in the control group, except that the abolition does not bring back to life the previously destroyed jobs, since we track survivors only. As a result, the former treatment group features a missing mass of marginal matches with respect to the REBP surplus shock. This gap is indicated by a parallel gap between the surviving matches and the zero-joint-surplus line. By contrast, these low-surplus jobs continue to be present in the former control group, depicted in Panel (c.C).

The testable prediction characterizing the Coasean view is that the former treated group should exhibit attenuated sensitivities to any post-REBP surplus shocks compared to the control group. Figure 11 Panels (b.C) and (c.C) illustrate this feature in form of shifts in the worker component of surplus to the west, which moves the job down the ranking of iso-joint-surplus lines. But as long as the subsequent shock is smaller than the REBP shocks, it will not entail any separations. Importantly, in the Coasean setting, the same resilience would emerge with an equally sized firm surplus - i.e. southward - decline. Therefore due to efficient (re)bargaining and hence joint surplus serving as the allocative concept, the missing mass of low-joint-surplus matches emerging from REBP henceworth attenuates the formerly treated REBP survivors' separations in response to surplus shocks of either kind. Just as in Coasean bargaining initial endowments are irrelevant to achieving efficient outcomes, in the job setting the initial incidence of shocks (between workers and firms) is irrelevant. Such "fungibility" of the incidence of surplus shifts is broken in the non-Coasean setting in Section 6, and foreshadowed in the lower panels of Figure 11 .

Note that in Figure 11 Panels (b.C) and (c.C) we are implicitly considering the case immediately following the abolition of REBP, when idiosyncratic shocks have not yet reshuffled the surplus distribution (although during REBP such shocks have hit), generating a clean missing mass of marginal matches. We discuss and relax this assumption in the formal model below, by permitting such idiosyncratic shocks. 


\subsection{Formal Model of the Research Design}

The full model below formalizes the effect of REBP and its abolition within the general Coasean model of jobs.

During-REBP Separation Behavior Separations (during [after] REBP denoted by $\delta[\Delta]$ ) occur if joint surplus were to turn negative, either due to aggregate shocks denoted by $\varepsilon$ (e.g., $\varepsilon_{b}^{W \prime}$ from the shift in UI benefits) or idiosyncratic shocks (health, productivity, amenities,...). Denote by $k\left(\mathbf{V}^{\prime} \mid \mathbf{V}\right)$ the Markov process governing the transition of job values into REBP and by $K\left(\mathbf{V}^{\prime \prime} \mid \mathbf{V}^{\prime}\right)$ the Markovian transition out of REBP, into the post-REBP period. We define $\widetilde{S}\left(\mathbf{V}^{\prime}\right)$ as the short-hand for the surplus level gross of a given aggregate surplus shifter, such that, for an aggregate shock $-\varepsilon^{\prime}<0, \widetilde{S}\left(\mathbf{V}^{\prime}, \varepsilon^{\prime}=0\right)=S\left(\mathbf{V}^{\prime}, \varepsilon^{\prime}\right)-\varepsilon^{\prime}$. For REBP, $\varepsilon^{\prime}=\varepsilon_{b}^{W^{\prime}}$, and hence separations in the treatment [control] group $Z=1[=0]$ are:

$$
\delta^{Z}=\int_{\mathbf{V}} \underbrace{\int_{\mathbf{V}^{\prime}} \mathbb{1}\left(\widetilde{S}\left(\mathbf{V}^{\prime}\right)<Z \times \varepsilon_{b}^{W \prime}\right) k\left(\mathbf{V}^{\prime} \mid \mathbf{V}\right) d \mathbf{V}^{\prime}}_{\equiv \widetilde{\mathbb{d}}\left(\mathbf{V}, Z \times \varepsilon_{b}^{W \prime}\right)} f^{Z}(\mathbf{V}) d \mathbf{V} .
$$

where $\widetilde{d}$ is a slight modification of $\mathbb{d}$ defined in Equation $(10)$ to a gross-surplus concept with separate aggregate shocks, and $f^{Z}($.$) denotes the distribution prevailing at the onset of REBP,$ where we will assume that initial distributions are the same across groups $f^{0}()=.f^{1}($.$) . In$ Appendix Table A.1. we empirically support this condition for observable characteristics. By contrast, $f_{\text {post }}^{Z}($.) will denote post-REBP distributions that will naturally diverge due to REBP, not only in terms of surplus, but also in terms of some direct observables as captured by the complier analysis in Section 3.2

In this framework, the treatment effect estimated in Section 3 corresponds to:

$$
\begin{aligned}
\delta^{1}-\delta^{0} & =\int_{\mathbf{V}} \int_{\mathbf{V}^{\prime}} \mathbb{1}\left(0 \leq \widetilde{S}\left(\mathbf{V}^{\prime}\right)<\varepsilon_{b}^{W \prime}\right) k\left(\mathbf{V}^{\prime} \mid \mathbf{V}\right) d \mathbf{V}^{\prime} f^{0}(\mathbf{V}) d \mathbf{V}=\int_{\mathbf{V}} \int_{\mathbf{V}^{\prime} \in M^{\prime}} k\left(\mathbf{V}^{\prime} \mid \mathbf{V}\right) d \mathbf{V}^{\prime} f^{0}(\mathbf{V}) d \mathbf{V} \\
& =\int_{\mathbf{V}}\left[\widetilde{d}\left(\mathbf{V}, \varepsilon_{b}^{W \prime}\right)-\widetilde{d}(\mathbf{V}, 0)\right] f^{0}(\mathbf{V}) d \mathbf{V},
\end{aligned}
$$

where the last line clarifies that the difference in separation rates comes from different thresholds (the gross-of-REBP surplus in the treated regions needs to meet a higher bar) and not from different pre-REBP distributions between the treated and the control regions (which instead we assume to be the same). The marginal jobs extracted by REBP make up set $M^{\prime}=\left\{\mathbf{V}^{\prime}: 0 \leq \widetilde{S}\left(\mathbf{V}^{\prime}\right)<\varepsilon_{b}^{W \prime}\right\}$. These jobs correspond to those indicated by the red polka-dot pattern in Figure 5 (and the cut-outs in contour maps in Figure 11 (upper panels)). Figure 5 Panel (a) visualizes these surplus ranges, plotting the hypothetical distribution of gross-of-REBP surplus $\widetilde{S}\left(\mathbf{V}^{\prime}\right)$ at the onset of REBP. Our model makes no assumption on the origin of this distribution through surplus evolution $k\left(\mathbf{V}^{\prime} \mid \mathbf{V}\right)$. The surplus distribution is partitioned into: (i) jobs that separate even in the control group - 
fraction $\delta^{0}$ of the total mass at the onset of REBP (light-blue solid pattern); (ii) marginal jobs that separate due to REBP - fraction $\delta^{1}-\delta^{0}$ (red polka-dot pattern); and (iii) infra-marginal jobs that don't separate even with REBP - fraction $1-\delta^{1}$ (green dashed pattern).

REBP-Induced Truncation of the Surplus Distribution After the abolition of REBP, the program has truncated the treatment group's joint-surplus distribution below $\varepsilon_{b}^{W \prime}$. Hence, while the wider set of surviving jobs in the control group is $J^{\prime}=\left\{\mathbf{V}^{\prime}: \widetilde{S}\left(\mathbf{V}^{\prime}\right) \geq 0\right\}$, in the treatment group, the entire mass of survivors is concentrated in the inframarginal jobs, $V^{\prime} \in\left(J^{\prime} \backslash M^{\prime}\right)$. ${ }^{34}$

In schematic Figure 5. Panels (b) and (c) plot the corresponding surplus distributions right at the end of REBP - when $K\left(\mathbf{V}^{\prime \prime} \mid \mathbf{V}^{\prime}\right)$ has not yet kicked in. Panel (b) plots the treatment group, where all end-of-REBP survivors are inframarginal jobs ( $1-\delta^{1}$ of the original mass). In the control group, Panel (c) shows that among the survivors ( $1-\delta^{0}$ of pre-REBP jobs), fraction $\frac{\delta^{1}-\delta^{0}}{1-\delta^{0}}=18.3 \%$ are marginal, and $\frac{1-\delta^{1}}{1-\delta^{0}}=81.7 \%$ are inframarginal.

Post-Abolition Separation Behavior We denote post-abolition-of-REBP functions with capital letters, namely $\Delta$ for $\delta, \mathbb{D}$ for $\mathbb{d}$, and $K$ for $k$. Post-REBP aggregate shocks and job value factors are denoted by " rather than'. The post-REBP separation behavior of the formerly treated and control groups can be formalized by considering aggregate (common to both groups) worker and firm surplus shocks $\varepsilon^{W^{\prime \prime}}$ and $\varepsilon^{F^{\prime \prime}}$, which we combine into a joint-surplus shock $\varepsilon^{\prime \prime}=\varepsilon^{W^{\prime \prime}}+\varepsilon^{F^{\prime \prime}}$. Post-REBP, these shocks lead to the following separation rates in the treatment [control] group $Z=1[=0]$ :

$$
\Delta^{Z}=\int_{\mathbf{V}^{\prime}} \underbrace{\int_{\mathbf{V}^{\prime \prime}} \mathbb{1}\left(\widetilde{S}\left(\mathbf{V}^{\prime \prime}\right)<\varepsilon^{\prime \prime}\right) K\left(\mathbf{V}^{\prime \prime} \mid \mathbf{V}^{\prime}\right) d \mathbf{V}^{\prime \prime}}_{\equiv \widetilde{\mathbb{D}}\left(\mathbf{V}^{\prime}, \varepsilon^{\prime \prime}\right)} f_{\text {post }}^{Z}\left(\mathbf{V}^{\prime}\right) d \mathbf{V}^{\prime} .
$$

Post-REBP, differences in separation rates will arise from differences in $f_{\text {post }}^{Z}$, the densities of job qualities between the treatment and the control groups, due to the selective separations induced by REBP (rather than from differences in aggregate shocks and thresholds $\varepsilon^{W^{\prime \prime}}$ and $\varepsilon^{F^{\prime \prime}}$, which in turn we here assume to the same across the groups, hence unlike during REBP, which shifted thresholds $\left.Z \times \varepsilon_{b}^{W \prime}\right)$ :

$$
\Delta^{1}-\Delta^{0}=\int_{\mathbf{V}^{\prime}} \widetilde{\mathbb{D}}\left(\mathbf{V}^{\prime}, \varepsilon^{\prime \prime}\right)\left[f_{\text {post }}^{1}\left(\mathbf{V}^{\prime}\right)-f_{\text {post }}^{0}\left(\mathbf{V}^{\prime}\right)\right] d \mathbf{V}^{\prime}
$$

${ }^{34}$ The density $f_{\text {post }}^{1}\left(\mathbf{V}^{\prime}\right)$ is zero for the marginal jobs, while the inframarginal REBP survivors reflect the (conditional) distribution in the control group starting from truncation point $\varepsilon_{b}^{W \prime}$ :

$$
f_{\text {post }}^{1}\left(\mathbf{V}^{\prime}\right)= \begin{cases}0 & \text { if } \quad \mathbf{V}^{\prime} \notin\left(J^{\prime} \backslash M^{\prime}\right) \Leftrightarrow \widetilde{S}\left(\mathbf{V}^{\prime}\right)<\varepsilon_{b}^{W \prime} \\ \frac{f_{\text {post }}^{0}\left(\mathbf{V}^{\prime}\right)}{1-\int_{\mathbf{V} \in M^{\prime}} f_{\text {post }}^{0}\left(\mathbf{V}^{\prime}\right) d \mathbf{V}^{\prime}} & \text { if } \quad \mathbf{V}^{\prime} \in\left(J^{\prime} \backslash M^{\prime}\right) \Leftrightarrow \widetilde{S}\left(\mathbf{V}^{\prime}\right) \geq \varepsilon_{b}^{W \prime} .\end{cases}
$$


We now derive the separation rate of the former treatment group by replacing its densities as truncated versions of the control group's, as following Equation (13):35

$$
\begin{aligned}
\Delta^{1} & =\int_{\mathbf{V}^{\prime}} \widetilde{\mathbb{D}}\left(\mathbf{V}^{\prime}, \varepsilon^{\prime \prime}\right) f_{\text {post }}^{1}\left(\mathbf{V}^{\prime}\right) d \mathbf{V}^{\prime} \\
& =\int_{\mathbf{V}^{\prime} \in M^{\prime}} \widetilde{\mathbb{D}}\left(\mathbf{V}^{\prime}, \varepsilon^{\prime \prime}\right) f_{\text {post }}^{1}\left(\mathbf{V}^{\prime}\right) d \mathbf{V}^{\prime}+\int_{\mathbf{V}^{\prime} \notin M^{\prime}} \widetilde{\mathbb{D}}\left(\mathbf{V}^{\prime}, \varepsilon^{\prime \prime}\right) f_{\text {post }}^{1}\left(\mathbf{V}^{\prime}\right) d \mathbf{V}^{\prime} \\
& =0+\int_{\mathbf{V}^{\prime} \notin M^{\prime}} \widetilde{\mathbb{D}}\left(\mathbf{V}^{\prime}, \varepsilon^{\prime \prime}\right)\left[f_{\text {post }}^{0}\left(\mathbf{V}^{\prime}\right) \frac{1-\delta^{0}}{1-\delta^{1}}\right] d \mathbf{V}^{\prime} \\
& =\frac{1-\delta^{0}}{1-\delta^{1}}\left[\int_{\mathbf{V}^{\prime} \notin M^{\prime}} \widetilde{\mathbb{D}}\left(\mathbf{V}^{\prime}, \varepsilon^{\prime \prime}\right) f_{\text {post }}^{0}\left(\mathbf{V}^{\prime}\right) d \mathbf{V}^{\prime} \pm \int_{\mathbf{V}^{\prime} \in M^{\prime}} \widetilde{\mathbb{D}}\left(\mathbf{V}^{\prime}, \varepsilon^{\prime \prime}\right) f_{\text {post }}^{0}\left(\mathbf{V}^{\prime}\right) d \mathbf{V}^{\prime}\right] \\
& =\frac{1-\delta^{0}}{1-\delta^{1}}\left[\Delta^{0}-\int_{\mathbf{V}^{\prime} \in M^{\prime}} \widetilde{\mathbb{D}}\left(\mathbf{V}^{\prime}, \varepsilon^{\prime \prime}\right) f_{\text {post }}^{0}\left(\mathbf{V}^{\prime}\right) d \mathbf{V}^{\prime}\right] .
\end{aligned}
$$

The intuition is straightforward: modulo re-scaling by $\frac{1-\delta^{0}}{1-\delta^{1}}$ (since, post-REBP, the fraction of original jobs that remain in the treatment and in the control group is different), the separation behavior of the treatment group $\left(\Delta^{1}\right)$ is the same as that of the control group $\left(\Delta^{0}\right)$ except for the contribution of marginal matches $\left(\mathbf{V}^{\prime} \in M^{\prime}\right)$ to the separation behavior of the control group, which the expression nets out.

In order to map Equation (17) into an empirically tangible object, we now put some structure on $K\left(\mathbf{V}^{\prime \prime} \mid \mathbf{V}^{\prime}\right)$.

Post-Abolition Idiosyncratic Shocks $K\left(\mathbf{V}^{\prime \prime} \mid \mathbf{V}^{\prime}\right)$ Up until now we have not made any assumption on the processes underlying the evolution of job surplus, $k\left(\mathbf{V}^{\prime} \mid \mathbf{V}\right)$ and $K\left(\mathbf{V}^{\prime \prime} \mid \mathbf{V}^{\prime}\right)$ - neither during REBP, nor for separations after the abolition. Two extreme cases are now presented below: perfect persistence as well as immediate reshuffling of idiosyncratic surplus. Importantly, in both cases, the evolution of surplus during the five-year REBP period $k\left(V^{\prime} \mid \boldsymbol{V}\right)$ is left fully general. We only specify the Markov process for right after REBP is abolished in 1993, namely $K\left(V^{\prime \prime} \mid V^{\prime}\right)$, so that this assumption only covers a shorter time horizon than the original REBP period.

Case I: Perfect Persistence This case permits fully general pre-abolition evolution $k\left(\mathbf{V}^{\prime} \mid \mathbf{V}\right)$. But it assumes that right after the abolition of REBP, specifically between the abolition period and the next period, the underlying job surplus is persistent - as the contour maps in Section 5.2 above assumed. The sensibility of this assumption naturally depends on the given time interval from the abolition to the time we measure separations in the data; our most conservative horizon is

${ }^{35}$ Specifically, from Equation $\left[13\right.$, for $\mathbf{V}^{\prime} \notin M^{\prime}$ :

$$
f_{\text {post }}^{1}\left(\mathbf{V}^{\prime}\right)=\frac{f_{\text {post }}^{0}\left(\mathbf{V}^{\prime}\right)}{1-\int_{\mathbf{V}^{\prime} \in M^{\prime}} f_{\text {post }}^{0}\left(\mathbf{V}^{\prime}\right) d \mathbf{V}^{\prime}}=\frac{f_{\text {post }}^{0}\left(\mathbf{V}^{\prime}\right)}{1-\frac{\delta^{1}-\delta^{0}}{1-\delta^{0}}}=f_{\text {post }}^{0}\left(\mathbf{V}^{\prime}\right) \frac{1-\delta^{0}}{1-\delta^{1}},
$$

where the second equality follows from the fact that the mass of marginal jobs (i.e. $\mathbf{V}^{\prime} \in M^{\prime}$ ) in the control group is $\frac{\delta^{1}-\delta^{0}}{1-\delta^{0}}$. 
one single year following the abolition. Formally, the post-abolition transition matrix is now the identity matrix: $K\left(\mathbf{V}^{\prime \prime} \mid \mathbf{V}^{\prime}\right)=1$ if $\mathbf{V}^{\prime \prime}=\mathbf{V}^{\prime}$ and 0 otherwise; as a result, $\widetilde{\mathbb{D}}\left(\mathbf{V}^{\prime}, \varepsilon^{\prime \prime}\right)=\int_{\mathbf{V}^{\prime \prime}} \mathbb{1}\left(\widetilde{S}\left(\mathbf{V}^{\prime \prime}\right)<\right.$ $\left.\varepsilon^{\prime \prime}\right) K\left(\mathbf{V}^{\prime \prime} \mid \mathbf{V}^{\prime}\right) d \mathbf{V}^{\prime \prime}=\mathbb{1}\left(\widetilde{S}\left(\mathbf{V}^{\prime}\right)<\varepsilon^{\prime \prime}\right)$.

It is useful to distinguish two cases: $\varepsilon^{\prime \prime} \leq \varepsilon_{b}^{W^{\prime}}$, and $\varepsilon^{\prime \prime}>\varepsilon_{b}^{W_{\prime}}$. In case $\varepsilon^{\prime \prime} \leq \varepsilon_{b}^{W^{\prime}}$, it follows from the definition of $\Delta^{1}$ in Equation (14) that only marginal matches separate i.e. $\Delta^{0}=\int_{\mathbf{V}^{\prime} \in M^{\prime}} \widetilde{\mathbb{D}}\left(\mathbf{V}^{\prime}, \varepsilon^{\prime \prime}\right) f_{\text {post }}^{0}\left(\mathbf{V}^{\prime}\right) d \mathbf{V}^{\prime}$, and therefore ${ }^{36}$

$$
\Delta_{\varepsilon^{\prime \prime} \leq \varepsilon_{b}^{W}}^{1}=0
$$

In case $\varepsilon^{\prime \prime}>\varepsilon_{b}^{W \prime}$, all marginal matches separate in the control group and more, and so $\widetilde{\mathbb{D}}\left(\mathbf{V}^{\prime}, \varepsilon^{\prime \prime}\right)=$ $1 \forall \mathbf{V}^{\prime} \in M^{\prime}$, and $\Delta^{0}>\int_{\mathbf{V}^{\prime} \in M^{\prime}} \widetilde{\mathbb{D}}\left(\mathbf{V}^{\prime}, \varepsilon^{\prime \prime}\right) f_{\text {post }}^{0}\left(\mathbf{V}^{\prime}\right) d \mathbf{V}^{\prime}$. Hence, for this case, Equation (17) becomes:

$$
\begin{aligned}
\Delta_{\varepsilon^{\prime \prime}>\varepsilon_{b}^{W}}^{1,} & =\frac{1-\delta^{0}}{1-\delta^{1}}\left[\Delta^{0}-\int_{\mathbf{V}^{\prime} \in M^{\prime}} f_{\text {post }}^{0}\left(\mathbf{V}^{\prime}\right) d \mathbf{V}^{\prime}\right] \\
& =\frac{1-\delta^{0}}{1-\delta^{1}}\left[\Delta^{0}-\frac{\delta^{1}-\delta^{0}}{1-\delta^{0}}\right]
\end{aligned}
$$

where $\frac{\delta^{1}-\delta^{0}}{1-\delta^{0}}$ is the fraction of marginal jobs in the control group, as discussed above.

Putting the two cases together, for the full range of aggregate shocks $\varepsilon^{\prime \prime}$ - which are unobserved but sufficiently revealed through realized control group post-REBP separation rate $\Delta^{0}$ - we obtain the model-predicted $\Delta^{1}$ as a function of $\Delta^{0}$, piece-wise linear with slopes and kink positions given by $\left(\delta^{0}, \delta^{1}\right)$ :

$$
\Delta^{1}\left(\Delta^{0}\left(\varepsilon^{\prime \prime}\right), \delta^{0}, \delta^{1}\right)=\max \left\{0, \frac{1-\delta^{0}}{1-\delta^{1}}\left[\Delta^{0}\left(\varepsilon^{\prime \prime}\right)-\frac{\delta^{1}-\delta^{0}}{1-\delta^{0}}\right]\right\} .
$$

Figure 10 Panel (b) illustrates the relationship between post-REBP treatment group against control group separations for the persistence version of the Coasean model formalized by Equation (18) for an example truncation point and surplus distribution, in form of the red dashed line, along with other variants the paper derives later.

As long as control group post-REBP separation rate $\Delta^{0}$ is lower than the fraction of marginal matches $\frac{\delta^{1}-\delta^{0}}{1-\delta^{0}}$, no separations should occur in the treatment group, simply because these matches are missing. Once control group separations cross that threshold, separations commence, and with a slope steeper than one, $\frac{1-\delta^{0}}{1-\delta^{1}}$, because the incremental separator count is over a smaller count of survivors in the formerly treated group, and both groups will have separation rates of $100 \%$ if all control jobs dissolve. Similarly, if the initial REBP treatment effect was zero, the curve would trace out a 45 degree line $\Delta^{1}=\Delta^{0}$. In that sense, the design has power if the initial treatment effect during REBP was large - shifting the kink far to the right away from zero on the x-axis.

That is, the revealed-preference treatment/control group approach makes empirically and

\footnotetext{
${ }^{36}$ To see this, note that under the assumption of perfect persistence, $\Delta^{0}=\int_{\mathbf{V}^{\prime}} \mathbb{1}\left(\widetilde{S}\left(\mathbf{V}^{\prime}\right)<\varepsilon^{\prime \prime}\right) f_{\text {post }}^{0}\left(\mathbf{V}^{\prime}\right) d \mathbf{V}^{\prime}$. If $\varepsilon^{\prime \prime} \leq$ $\varepsilon_{b}^{W \prime}$, all the separations come from $\mathbf{V}^{\prime}$ such that $\mathbb{1}\left(\widetilde{S}\left(\mathbf{V}^{\prime}\right)<\varepsilon_{b}^{W \prime}\right)$, which are $\mathbf{V}^{\prime} \in M^{\prime}$; therefore $\Delta^{0}=\int_{\mathbf{V}^{\prime}} \mathbb{1}\left(\widetilde{S}\left(\mathbf{V}^{\prime}\right)<\right.$ $\left.\varepsilon^{\prime \prime}\right) f_{\text {post }}^{0}\left(\mathbf{V}^{\prime}\right) d \mathbf{V}^{\prime}=\int_{\mathbf{V}^{\prime} \in M^{\prime}} \mathbb{1}\left(\widetilde{S}\left(\mathbf{V}^{\prime}\right)<\varepsilon^{\prime \prime}\right) f_{\text {post }}^{0}\left(\mathbf{V}^{\prime}\right) d \mathbf{V}^{\prime}=\int_{\mathbf{V}^{\prime} \in M^{\prime}} \widetilde{\mathbb{D}}\left(\mathbf{V}^{\prime}, \varepsilon^{\prime \prime}\right) f_{\text {post }}^{0}\left(\mathbf{V}^{\prime}\right) d \mathbf{V}^{\prime}$
} 
quantitatively tractable the Coasean benchmark (with persistence) by reformulating the empirically elusive surplus concepts in form of observables $-\Delta^{1}, \Delta^{0}$ and $\left(\delta^{0}, \delta^{1}\right)$. These properties sufficiently encode the surplus concepts $S$ as well as shocks $\varepsilon_{b}^{W \prime}$ of REBP, and the post-abolition $\operatorname{shocks}\left(\varepsilon^{W \prime \prime}, \varepsilon^{F \prime \prime}\right)$.

Complementing the separation-rate-based illustration in Figure 10 Panel (b), we also include a surplus-based representation driving the separations in Panels (c) and (d), which again as a red dashed line plots the model-predicted comovement between the post-REBP separation rates of the treatment and control groups, but now both as a function of post-abolition worker surplus shifts $\varepsilon^{W \prime \prime}$ (Panel (a)) and firm surplus shifts $\varepsilon^{W \prime \prime}$ (Panel (b)). In the Coasean setting (but not in the non-Coasean alternative discussed in Section 6 and also traced out in the figure), the initial incidence on firms and workers is inconsequential, since only joint surplus, stemming from the sum of the two shocks $\varepsilon^{\prime \prime}=\varepsilon^{W \prime \prime}+\varepsilon^{F \prime}$, matters. Therefore, either shock type leaves the formerly treated group's separations flat at zero - up until the truncation point of surplus equal to the REBP surplus threshold $\varepsilon_{b}^{W \prime}$, from which point on they turn positive with a steeper slope than in the control group, eventually meeting the control group at $100 \%$ for very large surplus shocks. We have therefore now formally derived of the resilience intuitively described in the contour maps in Figure 11 (upper panels).

Interim Empirical Evaluation of Coasean Benchmark: Cohort Graphs To gauge the gap between the data and a benchmark model, we compute and provide the predicted separations following a strictly interpreted Coasean view with perfect persistence in job surplus as presented in Equation (18). Specifically, for each cohort, we collect the separation rates in the control and REBP regions to proxy for $\left(\delta_{c}^{0}, \delta_{c}^{1}\right)$ (respectively the blue solid and red dashed lines respectively in Figure 2 Panel (a)), and feed in post-REBP cohort-specific separation rates from the peer cohorts in the control group $\Delta_{c}^{0}$ (blue solid line in Figure 6). The yellow dashed line in Figures 6 (levels) and 7 (differences) plots this predicted Coasean (perfect persistence) benchmark, along with the actually realized post-REBP separation rates by cohort for the treatment and control groups. We do so for the first four post-abolition years.

The figures confirm that the design has power. For instance, within the first year (Figure 6(a)), which covers the first post-abolition year through 1995, the benchmark model would predict zero separations in the formerly treated group for the majority of formerly treated cohorts, whereas the control group's actual post-REBP separation rate hovers around $20 \% 37$ This is because as Figure 2 Panels (a) and (b) clarify, $\frac{\delta_{c}^{1}-\delta_{c}^{0}}{1-\delta_{c}^{0}}$ i.e. the fraction of marginal jobs in the control group was even higher than the control group post-REBP separation rate, such that, post-REBP in the control group, only the marginal jobs are separating - which are not present in the treated group with persistence in job surplus.

In stark contrast to this Coasean benchmark, the empirical cohort gradient of the post-abolition separation rate in the formerly treated group follows a pattern that instead nearly perfectly co-

\footnotetext{
${ }^{37}$ Separations spike once a birth cohort turns 60 years old, the age of retirement for Austrian men in this period.
} 
incides with the post-abolition separation rate cohort gradient of the control group. This is true already at the shortest post-abolition time horizon, from 1994 to 1995, likely captured best by persistence in job-level surplus. Yet even over longer horizons, multiple years post-separation, the design retains power but the differences shrink (since $\Delta^{0}$ grows) as Figures 6(b) through (d) clarify. (On the other hand, at those multi-year horizons such as from 1994 to 1998, the assumption of persistence in idiosyncratic job-level surplus may serve as a weaker approximation.)

This Coasean benchmark clarifies that the absence of any attenuated separation responses whatsoever among the treated cohorts documented in Section 4 is quantitatively significant benchmarked against this prediction, because the effect induced by the REBP on separations was so dramatic (Section 3 and Figures 2 and 3 .

Case II: Perfect Reshuffling Next, we clarify the conditions required for the Coasean framework to rationalize the observed pattern: $\Delta^{1}=\Delta^{0}$. Since we will compare $\Delta^{0}$ and $\Delta^{1}$ across cohorts (and for an even wider range across an industry-occupation cells in an additional design below in Section 5.4.3, we look for the condition that is capable of delivering $\Delta^{1}\left(\varepsilon^{\prime \prime}\right)=\Delta^{0}\left(\varepsilon^{\prime \prime}\right)$ for the entire range of post-REBP aggregate shocks $\varepsilon^{\prime \prime}$ hence captured by any level of $\Delta^{0} \in[0,1]$, and for any $\left(\delta_{0}, \delta_{1}\right)$ arising from the REBP determining the missing mass of low-surplus matches in the former control group. We show that this condition yields a particular assumption about the process governing idiosyncratic surplus evolution - which then renders inconsequential the extraction of marginal jobs during REBP, by reshuffling immediately and fully the surplus distribution, such that the former treatment and control groups reconverge despite the massive extraction of marginal, low-surplus jobs. This condition requires that individual jobs do not have any persistence in their job-level surplus and their ranking, such that even within just a year the "hole" left by REBP in the treatment group is fully filled again. Formally, the Coasean case requires the following condition for equality between the separation rates:

$$
\begin{aligned}
& \Delta^{1}\left(\varepsilon^{\prime \prime}, \delta_{0}, \delta_{1}\right)=\Delta^{0}\left(\varepsilon^{\prime \prime}, \delta_{0}, \delta_{1}\right)
\end{aligned}
$$

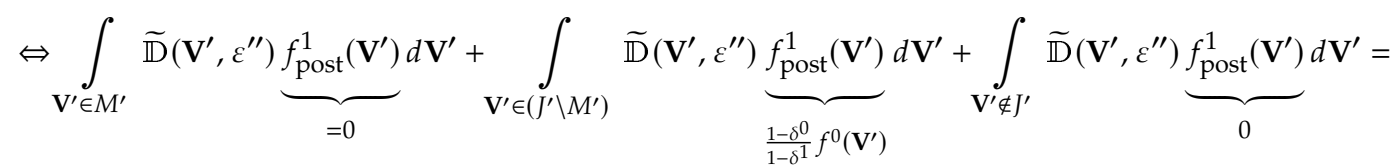

$$
\begin{aligned}
& \int_{\mathbf{V}^{\prime} \in M^{\prime}} \widetilde{\mathbb{D}}\left(\mathbf{V}^{\prime}, \varepsilon^{\prime \prime}\right) f_{\text {post }}^{0}\left(\mathbf{V}^{\prime}\right) d \mathbf{V}^{\prime}+\int_{\mathbf{V}^{\prime} \in\left(J^{\prime} \backslash M^{\prime}\right)} \widetilde{\mathbb{D}}\left(\mathbf{V}^{\prime}, \varepsilon^{\prime \prime}\right) f_{\text {post }}^{0}\left(\mathbf{V}^{\prime}\right) d \mathbf{V}^{\prime}+\int_{\mathbf{V}^{\prime} \notin J^{\prime}} \widetilde{\mathbb{D}}\left(\mathbf{V}^{\prime}, \varepsilon^{\prime \prime}\right) \underbrace{f_{\text {post }}^{0}\left(\mathbf{V}^{\prime}\right) d \mathbf{V}^{\prime}}_{0} \\
& \Leftrightarrow \int_{\mathbf{V}^{\prime} \in M^{\prime}} \widetilde{\mathbb{D}}\left(\mathbf{V}^{\prime}, \varepsilon^{\prime \prime}\right) f_{\text {post }}^{0}\left(\mathbf{V}^{\prime}\right)\left[\frac{1-\delta^{0}}{\delta^{1}-\delta^{0}}\right] d \mathbf{V}^{\prime}=\int_{\mathbf{V}^{\prime} \in\left(J^{\prime} \backslash M^{\prime}\right)} \widetilde{\mathbb{D}}\left(\mathbf{V}^{\prime}, \varepsilon^{\prime \prime}\right) f_{\text {post }}^{0}\left(\mathbf{V}^{\prime}\right)\left[\frac{1-\delta^{0}}{1-\delta^{1}}\right] d \mathbf{V}^{\prime} \\
& \Leftrightarrow \underbrace{\int_{\mathbf{V}^{\prime} \in M^{\prime}} \int_{\mathbf{V}^{\prime \prime}} \mathbf{1}\left\{\widetilde{S}\left(\mathbf{V}^{\prime \prime}\right)<\varepsilon^{\prime \prime}\right\} K\left(\mathbf{V}^{\prime \prime} \mid \mathbf{V}^{\prime}\right) d \mathbf{V}^{\prime \prime} \tilde{f}_{M}^{0}\left(\mathbf{V}^{\prime}\right) d \mathbf{V}^{\prime}}=\underbrace{\int_{\mathbf{V}^{\prime} \in\left(J^{\prime} \backslash M^{\prime}\right)} \int_{\mathbf{V}^{\prime \prime}} \mathbf{1}\left\{\widetilde{S}\left(\mathbf{V}^{\prime \prime}\right)<\varepsilon^{\prime \prime}\right\} K\left(\mathbf{V}^{\prime \prime} \mid \mathbf{V}^{\prime}\right) d \mathbf{V}^{\prime \prime} \tilde{f}_{I}^{0}\left(\mathbf{V}^{\prime}\right) d \mathbf{V}^{\prime}}, \\
& \text { Avg. sep. rate for the marginal jobs } \\
& \text { Avg. sep. rate of inframarginal jobs }
\end{aligned}
$$


where $\tilde{f}_{M}=f_{\text {post }}^{0}\left(\mathbf{V}^{\prime}\right)\left[\frac{1-\delta^{0}}{\delta^{1}-\delta^{0}}\right]$ is the density of the marginal jobs in the control group and $\tilde{f}_{I}^{0}=$ $f_{\text {post }}^{0}\left(\mathbf{V}^{\prime}\right)\left[\frac{1-\delta^{0}}{1-\delta^{1}}\right]$ is the density of the inframarginal jobs in the control group.

The derivation above shows that, to observe the same post-REBP separation rate in the treatment and the control group, the average post-REBP separation rate for the jobs in the marginal group $\left(\mathbf{V}^{\prime} \in M^{\prime}\right)$ must be the same as that for the jobs in the inframarginal group $\left(\mathbf{V}^{\prime} \in\left(J^{\prime} \backslash M^{\prime}\right)\right)$.

This condition is fulfilled if we have perfect reshuffling of job surpluses one period to the next (formally, a transition matrix with identical rows). 38 That is, perfect reshuffling of idiosyncratic job surplus can mask Coasean separations: if the economy featured no persistence in worker and firm surplus whatsoever (such that the idiosyncratic Markov process reshuffles the position of jobs into the same, stationary surplus distribution in each period), the economy would not actually feature a truncated distribution when REBP is abolished - despite the massive extraction of temporarilymarginal jobs from REBP. We will quantify the weight the data put on this extreme interpretation below in Section 5.4 in a simple structural model, and discuss this assumption and hence more broadly the capacity of the Coasean model to account for the empirical separation outcomes in Section $5.5^{39}$

\subsection{Structural Estimation of a "Mixed Model"}

Neither a full-persistence nor a perfect-reshuffling setting will fully describe empirical labor markets. To gauge the quantitative power of each extreme view, we present a structural estimation of a "mixed model" of the Coasean setting that asks which fraction of labor market cells must accord with the perfect-reshuffling variant to rationalize the observed patterns. We present two strategies: a simple reduced-form approach and a more formal approach based on a simple structural model. Both strategies reveal a formal estimate of the visually clear observed pattern that the Austrian labor market would need to be characterized by no persistence whatsoever in idiosyncratic job surplus to rationalize a Coasean framework. Confidence intervals bound this share from below at $89 \%$, with point estimates of $100 \%$ (or higher).

\subsubsection{Mixed Model}

Consider a labor market cell $i$ (cohort, or industry-occupation group) present in both REBP and non-REBP regions. We permit a given cell $i$ to operate according to either the full reshuffling

\footnotetext{
${ }^{38}$ Strictly speaking, for a given single surplus shock $\varepsilon^{\prime \prime}$ we do not need perfect reshuffling: the condition is that the average separation probabilities of marginal versus inframarginal jobs are the same. However, for the condition to hold globally i.e. for all $\varepsilon^{\prime \prime}$ surplus shocks and REBP treatment effects $\delta^{1}-\delta^{0}-$ a range we try to trace out with cohort cells but then also when splitting up the sample into industry-occupation cells with large variation in separation rates in an additional design below in Section 5.4.3-, perfect reshuffling (i.e. identical rows in the transition matrix) becomes necessary.

${ }^{39}$ Another assumption fulfills the condition at a particular point, but cannot account for the full set of findings: if all jobs have the same separation probability (say, some large negative (e.g., health) shock that is independent of the current job surplus), then the condition holds. But while tractable and intriguing, this assumption would be inconsistent with differential separation in the treated regions in response to REBP (i.e. our treatment effect in the first place) or across cells post-REBP.
} 
protocol in a Coasean setting or to the perfect-persistence benchmark. Share $\kappa$ is of the fullreshuffling type; share $1-\kappa$ is of the perfect-persistence type ${ }^{40}$ We estimate share $\kappa$. We do so by leveraging the structural relationships implied by our economic model of separations for either model, relating the post-REBP separation rates among cells in the former treatment group $Z=1$ i.e. $\Delta_{i}^{1}$ with the post-REBP separation rates among the peer cells in the control group $Z=0$, for a given cell-i-specific set of REBP separation rates $\left(\delta_{i}^{0}, \delta_{i}^{1}\right)$ (which we observe), as well as $i$ 's set of (not directly observed) post-REBP firm and worker shocks resulting in joint surplus shock $\varepsilon_{i}=\varepsilon_{i}^{W}+\varepsilon_{i}^{F}$ (which however is sufficiently revealed empirically by the post-REBP separation rate in the control group $\left.\Delta_{i}^{0}\right)$ :

$$
\Delta_{i}^{1}=\kappa \times \underbrace{\Delta_{i}^{0}}_{\text {Coasean: Reshuffling }}+(1-\kappa) \times \underbrace{\max \left\{0, \frac{1-\delta_{i}^{0}}{1-\delta_{i}^{1}} \cdot \Delta_{i}^{0}-\frac{\delta_{i}^{1}-\delta_{i}^{0}}{1-\delta_{i}^{1}}\right\}}_{\text {Coasean: Persistence }}+v_{i}
$$

since perfect reshuffling implies $\Delta_{i}^{1}=\Delta_{i}^{0}$ while perfect persistence implies that $\Delta_{i}^{1}$ follows the piecewise linear curve as predicted from Equation (18). $v_{i}$ captures errors related to, e.g., group-specific shocks.

\subsubsection{Reduced-Form Model with Cohort Variation}

Our first strategy estimates model (20) at the birth-year cohort level, comparing post-REBP separations by cohort between REBP and non-REBP regions (i.e. we collapse the data to the cohort and region level). That is, we essentially simply take the data points from cohort separation gradients plotted in Figures 6(levels) and7(differences) of the predicted Coasean (perfect persistence) benchmark, along with the original realized post-REBP separation rates by cohort for the treatment and control groups. We then let the data select weights on the yellow dashed line (perfect persistence) versus the blue solid line (perfect reshuffling). For simplicity, we here also approximate model (20) in this reduced form model while not imposing that the two models have weights adding up to one. Instead, we here estimate unconstrained coefficients $\alpha^{\kappa}$ as an estimate for $\kappa$ and $\alpha^{1-\kappa}$ as an estimate for $1-\kappa$.

Table 4 reports the estimates for various horizons after REBP. For the short run, 1995, the estimates imply an essential unit weight on the perfect reshuffling scenario as $\alpha^{\kappa}=1.027$ and an (insignificantly) negative weight on the perfect persistence scenario $\alpha^{1-\kappa}=-0.037$. In later years, when likely the power of the resilience prediction decreases, the estimate of weight $\alpha^{1-\kappa}$ becomes more negative and even statistically significant. That is, if we were in a Coasean world, we would fully reject any persistence of job surplus whatsoever, even already in the short run.

${ }^{40}$ An alternative interpretation of our setup is that we permit a share $\kappa$ of matches within a cell to be of the fullreshuffling type and the remainder of the perfect-persistence type. 


\subsubsection{Structural Estimation with Industry-by-Occupation Variation}

We now present a formal structural estimation of our model to estimate $\kappa$, by estimating Equation (20) with (i) richer variation at the industry-by-occupation level, (ii) accounting for the nonlinear nature of the specification (due to the max operator in Equation (18)), and (iii) providing a single estimate and confidence interval for $\kappa$ in each specification. (Our methodology could be easily applied to other cell categorizations.) This second estimation again reveals point estimates $\hat{\kappa}$ that are close to or even above 1. Even in our most conservative specification, the lower limit of the 95\% confidence interval for $\kappa$ is 0.890 , indicating that at least $89 \%$ of separations had to come from full reshuffling of job surplus for the data to be consistent with a Coasean setting.

Formally, we estimate $\kappa$ using non-linear least squares/GMM based on the moment condition $E\left[v_{i} \mid \Delta_{i}^{0}\right]=0$ in Equation (20). Intuitively, the parameter $\kappa$ is identified through the non-linearity in the relationship between $\Delta_{i}^{1}$ and $\Delta_{i}^{0}$ induced by the resilience to non-large shocks to surplus predicted by the Coasean model with persistence.

To proxy for $\Delta_{i}^{0}$, we use post-REBP separation rates among workers in the non-REBP region in the same industry-occupation cell who were not treated by the program and who thus still contain marginal jobs but that are exposed to plausibly similar industry-level surplus shocks. We use observed separation rates during REBP in the REBP and non-REBP regions to measure $\delta_{i}^{1}$ and $\delta_{i}^{0}$ in each industry-occupation cell.

Reduced-Form Evidence on Comovement of Separation Rates Before providing results of the structural estimation, we plot the reduced-form relationship between $\Delta_{i}^{1}$ and $\Delta_{i}^{0}$ at the industryby-occupation level in binned scatter plots in Figure 12 . The figure shows the relationship between post-REBP separation rates (at horizons ranging from 1995 to 1998) at the industry-by-occupation cell level in formerly treated cohorts (born between 1938 and 1943) in the REBP regions against separations rates in the same cohorts in non-REBP regions who did not experience the treatment. The relationship appears strong, positive, and linear. As a placebo benchmark, we also plot as outcome variable the separation rate in slightly younger, ineligible cohorts (born between 1943 and 1948). The relationship is just as strong and positive, thus providing evidence that the industryby-occupation cells in the different regions were affected by similar shocks to surplus. As Figure 12 reveals, there is no discernible difference between the slope of the relationship of separation behavior of formerly treated cohorts to their same-aged peers in the non-REBP region and the relationship in slightly younger cohorts. In particular, we do not observe evidence for resilience, i.e. lower separation rates, at low post-REBP separation rates in the non-REBP regions (which our structural model will then formally also link with variation in the cell-specific kink position).

Results of Estimation Table 5 reports estimates of $\kappa$ based on estimation of Equation (20) with non-linear least squares. Across specifications, we find estimates of $\kappa$ that are always above 1 in the non-linear least squares model. The smallest point estimate is 1.027 with variation at the four-digit level and considering post-abolition separations by 1995. Even the most conservative i.e. lowest 
$95 \%$ confidence interval for $\hat{\kappa}$ rules out $\hat{\kappa} \leq 0.89$, that fewer than $89 \%$ of cells in the Austrian labor market in our sample would need to be of the perfect-shuffling type to rationalize the data through the Coasean lens.

The table further reveals even larger estimates at longer horizons, e.g., with the confidence intervals excluding one when considering separations by 1998, according with intuition that full reshuffling becomes less implausible at multi-year horizons. However, even at the shortest horizon, considering separations by 1995 as outcome variable, we find that 100\% (lower 95\% confidence interval: $89 \%$ ) of the cells would need to be of the perfect reshuffling kind ${ }^{41}$

\subsection{Discussion of the Coasean Framework}

Some Persistence in Idiosyncratic Job Surplus The model and its estimation above have clarified that the Coasean model can only rationalize the evidence under narrow and strong assumptions, with no persistence whatsoever in job-level surplus, such that the extraction of a large amount of then-marginal jobs has no consequence for the formerly treated group's post-REBP job surplus distribution, which immediately converges to the control group's distribution. We believe that this assumption is unlikely to explain our results, although of course this property is difficult to assess empirically directly. First, such full convergence would be required already at the oneyear horizon. Second, the reform was large such that the idiosyncratic shocks would need to be accordingly large to replenish the mass of marginal matches: REBP increased separations by about 27\%; and as discussed above, REBP amounted to around $71 \%$ of the average worker's annual salary. Third, in Section 4, we conduct a complier analysis and in fact trace out some persistent observable attributes associated with the incremental REBP separators that distinguish them from their non-separating peers. Fourth, our sample contains older workers with high labor force attachment, whose baseline turnover is typically lower, perhaps plausibly suggesting that the amount of separation-relevant idiosyncratic shocks is, if anything, selected towards a stable and persistent surplus group.

Below in Section 6 we therefore study departures from the Coasean assumption to account for the observed patterns even in the presence of surplus persistence.

Market-Level and Spillovers Effects Our model appears to require that the abolition restore the surplus level of each formerly treated inframarginal survivor to the level of the respective control peer. That is, REBP only affects post-abolition separation behavior through the channel of the shift in the surplus composition (as marginal matches are now missing). A concern is that REBP could have affected the surplus distribution through potential separate channels that could offset and mask the predicted resilience 42

\footnotetext{
${ }^{41}$ We have also explored strategies to account for measurement error using methodology for nonlinear models developed in Li and Vuong (1998), Li (2002) and Schennach (2004), surveyed in Schennach (2012). These lead to qualitatively and quantitatively similar results.

${ }^{42}$ Relatedly, the demography literature distinguishes harvesting effects akin to our Coasean prediction - by which transitory negative shocks (e.g., heat waves) induce low-health individuals into exit and thereby reduce the subsequent
} 
Fortunately, due to the two control groups, our empirical double-difference design nets out and therefore implicitly permits but resolves - any market-level or spillover effects, even those that have some persistence. It does so specifically for the kinds of confounders that also affect the ineligible slightly younger workers (49 and younger) in the REBP regions, whom our second difference compares with the same younger birth cohorts in the non-REBP regions. For example, if REBP (or shocks correlated with REBP) had lowered firm investment, we would expect higher separations among slightly younger colleagues within the REBP regions (presumably close substitutes and in the same labor market). If norms to hire older workers had shifted persistently, we would expect 49 year old workers along with their 50 year old colleagues to be affected. A similar argument applies to the fact that REBP aimed to ameliorate the impact of restructuring the steel sector and thereby targeted perhaps overall slackening labor markets, and lower surplus matches. Plausibly, these market-wide trends did not discontinuously affect 49 year old workers than 51 year old workers, and hence are netted out by our difference-in-differences design.

The kind of concern our design does not rule out must therefore discontinuously affect eligible but not ineligible workers, and persistently so beyond the abolition of the program. Perhaps younger workers in REBP regions are more likely to retrain, making them more resilient to shocks compared to older (eligible) REBP workers, such that the ostensible absence of resilience may mask resilience among the old compared to if REBP had not happened. However, we would then expect young cohorts in REBP regions to be more resilient than their peers in the non-REBP regions. As another example, persistent wage effects of the workers' outside option boost could, in combination with wage rigidity, perhaps lead to subsequently higher separations masking resilience - but such a route to explain non-resilience already departs from the Coasean view by appealing to allocative wage rigidity 43 Relatedly, spillovers between eligible and ineligible cohorts within the REBP region may dilute the control group. Examples are search or labor demand spillovers (Lalive et al., 2011; Beaudry et al. 2012), replacement hiring (Mercan and Schoefer (forthcoming)), shifts in marginal products and wages (Jäger and Heining, 2016) - to the degree that these are persistent beyond the abolition of the policy (as in the hysteresis effects in Saez et al., 2019). However, these effects would then still need to show up in the differenced effect between the young across regions. These differences appear economically small or absent, supporting our design 44

mortality rate of the survivors - from scarring effects - by which the original shock may permanently lower the survivors' health going forward thereby working in the other direction in increasing subsequent mortality.

${ }^{43} \mathrm{We}$ cannot credibly study wage effects in the present context given the attrition (separation effects). Yet Jäger et al. (2018) document that in Austria, wages of stayers are insensitive to UI-induced boosts in workers' outside option, even for older workers such as our sample here, and during the 1980s.

${ }^{44}$ In principle, perhaps exactly the younger cohorts may in fact be expected to do worse in the REBP regions (even after dropping the steel sector), and hence their parallel behavior post-REBP with their non-REBP peers may be evidence of positive spillovers, such that the parallel age gradients may mask differential resilience among the old REBP cohorts. We expect any such attenuation to be mild given the already nearly perfect alignment between the young during REBP. Most importantly, the stable parallel behavior across the ineligible cohorts extends to even younger cohorts, as we confirmed in Appendix Figure A.3. making such differential aggregate trends unlikely to mask resilience among the eligible cohorts. In any case, such patterns would need to exactly offset the hump shape of the initial treatment effect in the separation-cohort gradient depicted in Figure 2 (b). 
Heterogeneous Sensitivity to the REBP Treatment Our model assumes homogeneous shifts in outside options and thus in the level of surplus - as a result of which the REBP marginal jobs are low-surplus jobs - rather than jobs whose surplus is more sensitive to the REBP treatment e.g. because valuation of REBP may be heterogeneous (in that the outside option increases by more for some workers than others).

One specific potential concern for our interpretation of (or a potential explanation for) the absence of post-abolition resilience is then that the workers responding to REBP by separating, are those workers that may have (initially and absent REBP) higher surplus. Then, the introduction and abolition of REBP would remove high-surplus workers - rather than generating a "hole" of low-surplus matches around the zero-surplus threshold. In principle, therefore, such a positive correlation between surplus valuation $\varepsilon_{b}^{W \prime}$ and gross-of-REBP surplus $\widetilde{S}$ could rationalize our findings even within a Coasean framework and even with persistence in job surplus.

To assess the relevance of this thought experiment, we sort workers into cells that are plausibly related to heterogeneous valuation of REBP i.e. in $\varepsilon_{b}^{W \prime}$. Our sorting criterion is a worker's risk of having a spell of nonemployment longer than one year ${ }^{45}$ This categorization should almost mechanically sort workers by a factor mediating the value of REBP because the program, by raising benefit duration from one to four years, shifted outside options depending on the worker's expected time in long-term nonemployment spells in excess of one year. To assign workers REBP-relevant long-term nonemployment risk scores to our sample employed at the onset of the REBP reform in 1988, we estimate a prediction model regressing realized nonemployment spell outcomes on pre-separation attributes, in the pre-reform sample of pre-reform period 46

Figure 13 plots three separation gradients as a function of this heterogeneity dimension, which we bin by quintile and then separately measure outcomes or estimate effects. First, the figure (in navy hollow squares and connected by the solid navy line) shows that the REBP treatment effect on separations during REBP was indeed substantially stronger among workers with a higher predicted risk of long-term nonemployment. In the lowest-risk quintile, the separation effects of REBP are relatively small at around 5 percentage points. The separation effects are markedly larger, at more than 20 percentage points, in the quintiles with the highest risk of long-term nonemployment ${ }^{47}$

Second, Figure 13 plots (in maroon solid circles, dotted line) the level of the separation rate (during REBP) for the control group. If workers in the highest bin of the valuation of REBP had absent REBP being active, as in the control group during REBP - higher surplus, then we would expect

\footnotetext{
${ }^{45}$ Other factors may be awareness of the policy, the capacity to quit, myopia, stigma costs from additional time unemployed.

${ }^{46}$ For all workers employed in 1982, we regress an indicator for whether they had a nonemployment spell of one year or longer on a rich set of covariates measured in 1982: industry fixed effects, an indicator for working in a white collar occupation, the local unemployment rate, and third degree polynomials in tenure and experience. We then take the estimated coefficients and predict the probability of having a nonemployment spell of one year or longer among the sample of workers employed in 1988 and create a predicted long-term nonemployment risk score. We then split workers into quintiles based on their long-term nonemployment risk score and estimate the effect of the REBP reform within each quintile in a fully interacted difference-in-differences regression taking out the baseline effects of being in an REBP-eligible cohort and residing in an REBP region both interacted with indicator variables for each risk quintile.

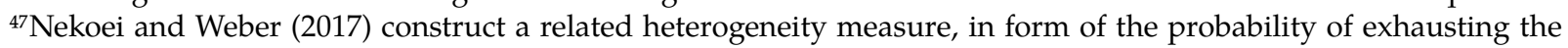
pre-reform benefit level with a focus on unemployed job seekers' search behavior, in the context of different reform.
} 
a steeply declining separation gradient as a function of the valuation variable. We do not detect such a gradient ${ }_{48}^{48}$ This result suggests that potential heterogeneity in workers' valuation of REBP is not positively associated with initial surplus.

Third, Figure 13 plots (in green hollow triangles and connected by the dashed green line) the post-REBP separation "treatment effect", i.e. the difference in the post-REBP separation rates within each risk cell. This slope is perfectly flat, indicating that even within the high valuation cells, where the treatment effect was the largest and where therefore high-valuation or low-surplus jobs were extracted, there is no resilience - an effect that is unlikely to be explained by these jobs having higher surplus on average (given the mostly flat red-square gradient for the REBP separation rate in the control regions).

\section{Which Non-Coasean Setting Can Rationalize the Findings?}

An alternative interpretation of the absence of resilience is that the separations were not Coasean. For example, frictions such as institutional constraints on selective layoffs or wage rigidity, may prevent the flexible (re-)bargaining and efficient-only separations underlying the Coasean result.

We now present one plausible non-Coasean alternative that can rationalize the full set of empirical findings, building on wage rigidity. We again start by presenting the core intuitions in stylized contour maps. We then move to the corresponding formal model. We again derive predictions by specifying two cases: one in which post-REBP separations in the former treatment and control groups track each other - namely if firm surplus shocks drive post-abolition separations - and one in which we would expect similar piece-wise linear resilience, namely if worker surplus shocks drive post-abolition separations. The non-Coasean model can hence rationalize the perfect comovement even with full persistence in idiosyncratic job surplus, if firm surplus shock drive most separations post-REBP.

\subsection{Intuitions from Contour Maps: Effects of REBP in the Non-Coasean Setting}

We now provide non-Coasean narrative of REBP and its aftermath in the non-Coasean setting, plotting contour maps of the density of the joint distribution of firm (y-axis) and worker (x-axis) net of wage surpluses in the lower panels of Figure 11. In the non-Coasean setting, wages are not efficiently (re-)bargained. As a result, the inequality conditions allocative for non-Coasean separations are the unilateral participation constraints of positive worker surplus and positive firm surplus i.e. the y-axis and x-axis. This contrasts with the Coasean diagonal line of zero joint surplus as in the upper panels discussed in Section 5.2

Figure 11 Panel (a.NC) illustrates that REBP improved worker's outside option i.e. lowered worker surplus, hence the treated jobs shift west. After REBP, Figure 11 Panel (a.NC) illustrates

${ }^{48} \mathrm{~A}$ priori, one might have even expected a strongly upward-sloping curve, whereas the empirical nearly flat line is perhaps explained by the sorting on the x-axis being unconditional of a separation, and because for consistency here we plot the most general notion of a job separation on the y-axis (rather than only those into nonemployment). 
that the former treatment group features a missing mass of low-worker-surplus matches. But the treatment group may retain a similar share of jobs with low firm surplus as the control group.

Figure 11Panels (b.NC) and (c.NC) depict the resulting post-REBP behavior in response to firm and worker shocks, separately for the former treatment group (Panel (b.NC)) and control group (Panel (c.NC)). The treatment group features resilience to negative post-REBP worker surplus shifts, but in response to firm surplus shocks, of which both groups may feature a similar share, separation responses can be accordingly similar.

For the sizable truncation along worker surplus to be quantitatively inconsequential for subsequent separation behavior, it must be that also the control group features hardly any separations to worker shocks post-REBP. This implies that absent REBP, the typical worker surplus must have been high to begin with (i.e. far to the right as depicted) ${ }^{49}$ And, REBP must have been large in order to reach deep into the worker surplus distribution to cause (otherwise inframarginal) workers to separate. In Section 6.4 below we discuss why these features are plausible for our sample and setting.

\subsection{Formal Model of the Research Design}

Non-Coasean Bargaining and Inefficient Separations The strong Coasean result of efficient separations arises from the assumption of flexible (re-)bargaining of compensation, from which joint job surplus stems as the sole allocative concept. However, a variety of potential real-world frictions, e.g. wage rigidity, may preclude such wage setting. In our model depicted in Figure 10 Panel (a), such frictions prevent the parties from moving towards a wage in the feasible-jobs frontier even though the job carries positive joint surplus, thereby shrinking the set of feasible jobs. Then, the Coasean allocation is not necessarily attainable, and inefficient separations can emerge. In this non-Coasean setting, we therefore think of wage $w$ as one additional job attribute that can evolve or be fixed, such that jobs are now characterized by $(w, \mathbf{V})$, and unilateral worker and firm surpluses $S^{W}\left(w, \mathbf{V}^{W}\right)$ and $S^{F}\left(w, \mathbf{V}^{F}\right)$ are allocative.

In consequence, with non-Coasean bargaining, a separation occurs if at least one of worker surplus or firm surplus turns negative at the given wage. Hence, the job-level separation probability is given by

$$
\mathbb{d}(w, \mathbf{V})=\int_{\left(w^{\prime}, \mathbf{V}^{\prime}\right)} \mathbb{1}(\underbrace{S^{W}\left(w^{\prime}, \mathbf{V}^{W \prime}\right)<0}_{\text {Quit }} \overbrace{\vee}^{\text {Mutual Sep.: }} \underbrace{S^{F}\left(w^{\prime}, \mathbf{V}^{F \prime}\right)<0}_{\text {Layoff }}) k\left(\left(w^{\prime}, \mathbf{V}^{\prime}\right) \mid(w, \mathbf{V})\right) d\left(w^{\prime}, \mathbf{V}^{\prime}\right),
$$

where separations can be labled as quits (negative worker surplus but positive firm surplus), layoffs (reversed), or mutual separations (both negative). The non-Coasean expression also formalizes that here the initial incidence of a shock matters for separations for lack of automatic Coasean rebargaining, such that worker and firm values are not "fungible."

\footnotetext{
${ }^{49}$ Alternatively, shocks to worker surplus may be smaller compared to firm shocks.
} 
Separation Effects from REBP REBP reduced worker surplus, as REBP directly shifted workers' (nonemployment) outside option. Any market-level effects that in turn affected firm outside options or inside values we net out with a control group in the data.

Formally, the two separation rates by treatment group $(Z=1)$ and control group $(Z=0)$ therefore occur to differential worker surplus cutoffs, albeit at the same firm cutoff (here again normalized to zero without loss of generality). Let $\widetilde{S}^{i}\left(w^{\prime}, \mathbf{V}^{\prime}\right)$ denote the surplus of party $i \in\{W, F\}$ gross of a given aggregate shock, e.g. during REBP again the REBP worker surplus shifter $\varepsilon_{b}^{W^{\prime}}$. We thus have:

$$
\begin{aligned}
\delta^{Z}= & \int_{(w, \mathbf{V})} \underbrace{\int_{\left(w^{\prime}, \mathbf{V}^{\prime}\right)} \mathbb{1}\left(\widetilde{S}^{W}\left(w^{\prime}, \mathbf{V}^{W \prime}\right)<Z \times \varepsilon_{b}^{W \prime} \vee \widetilde{S}^{F}\left(w^{\prime}, \mathbf{V}^{F \prime}\right)<0\right) k\left(\left(w^{\prime}, \mathbf{V}^{\prime}\right) \mid(w, \mathbf{V})\right) d\left(w^{\prime}, \mathbf{V}^{\prime}\right)}_{\equiv \widetilde{\mathbb{d}}\left(w, \mathbf{V} ; Z \times \varepsilon_{b}^{W \prime}, 0\right)} \\
& f^{Z}(w, \mathbf{V}) d(w, \mathbf{V}),
\end{aligned}
$$

where $\widetilde{\mathbb{d}}$ is a slight modification of $\mathbb{d}$ defined in Equation (21).

REBP therefore pushed the following mass of jobs initially viable in 1988 into quit or layoff (or both) territory of negative unilateral surpluses, where again $f^{Z}$ (.) denotes the pre-REBP initial surplus distribution:

$$
\begin{aligned}
\delta^{1}-\delta^{0}= & \int_{(w, \mathbf{V})} \int_{\left(w^{\prime}, \mathbf{V}^{\prime}\right)} \mathbb{1}\left(0 \leq \widetilde{S}^{W}\left(w^{\prime}, \mathbf{V}^{W \prime}\right)<\varepsilon_{b}^{W \prime} \wedge \widetilde{S}^{F}\left(w^{\prime}, \mathbf{V}^{F \prime}\right) \geq 0\right) \\
& k\left(\left(w^{\prime}, \mathbf{V}^{\prime}\right) \mid(w, \mathbf{V})\right) d\left(w^{\prime}, \mathbf{V}^{\prime}\right) f^{Z}(w, \mathbf{V}) d(w, \mathbf{V}) \\
& =\int_{(w, \mathbf{V})} \int_{\left(w^{\prime}, \mathbf{V}^{\prime}\right) \in M^{\prime N C}} k\left(\left(w^{\prime}, \mathbf{V}^{\prime}\right) \mid(w, \mathbf{V})\right) d\left(w^{\prime}, \mathbf{V}^{\prime}\right) f^{Z}(w, \mathbf{V}) d(w, \mathbf{V}) \\
& =\int_{(w, \mathbf{V})}\left[\widetilde{d}\left(w, \mathbf{V} ; \varepsilon_{b}^{W \prime}, 0\right)-\widetilde{d}(w, \mathbf{V} ; 0,0)\right] f^{0}(w, \mathbf{V}) d(w, \mathbf{V}) .
\end{aligned}
$$

That is, the incremental jobs destroyed by REBP had low worker surplus, between 0 and $\varepsilon_{b}^{W \prime}$, making up the set of marginal-to-REBP jobs $M^{\prime N C}=\left\{\left(w^{\prime}, \mathbf{V}^{\prime}\right): 0 \leq \widetilde{S}^{W}\left(w^{\prime}, \mathbf{V}^{W \prime}\right)<\varepsilon_{b}^{W \prime} \wedge \widetilde{S}^{F}\left(w^{\prime}, \mathbf{V}^{F \prime}\right) \geq 0\right\}$. By contrast, the firm surplus of these jobs were positive (and moreover need not have been low, unless the two are very correlated).

REBP-Induced Truncation of the Surplus Distribution As a result of REBP, right after the abolition, the treatment group therefore features a missing mass of marginal matches between 0 and $\varepsilon_{b}^{W \prime}$, making up the set of marginal-to-REBP jobs $M^{\prime N C}=\left\{\left(w^{\prime}, \mathbf{V}^{\prime}\right): 0 \leq \widetilde{S}^{W}\left(w^{\prime}, \mathbf{V}^{W \prime}\right)<\right.$ $\left.\varepsilon_{b}^{W \prime} \wedge \widetilde{S}^{F}\left(w^{\prime}, \mathbf{V}^{F^{\prime}}\right) \geq 0\right\}$ with low worker but not necessarily low firm surplus. By contrast, the distribution of surpluses in the control group remains a larger set $J^{\prime N C}=\left\{\left(w^{\prime}, \mathbf{V}^{\prime}\right): \widetilde{S}^{W}\left(w^{\prime}, \mathbf{V}^{W \prime}\right) \geq\right.$ 
$\left.0 \wedge \widetilde{S}^{F}\left(w^{\prime}, \mathbf{V}^{F \prime}\right) \geq 0\right\}$, still containing the low worker-surplus jobs $M^{\prime N C} 50$

Post-Abolition Separation Behavior We again define the post-REBP separation rate of treatment [control] group $Z=1[=0]$ as a function of common worker and firm shocks $\varepsilon^{W^{\prime \prime}}$ and $\varepsilon^{F^{\prime \prime}}$ (which we can now, in contrast to the Coasean model, no longer collapse into a joint surplus shock $\varepsilon^{\prime \prime}$ as shocks are no longer fungible):

$$
\begin{aligned}
\Delta^{Z}= & \int_{\left(w^{\prime}, \mathbf{V}^{\prime}\right)} \underbrace{\int_{\left(w^{\prime \prime}, \mathbf{V}^{\prime \prime}\right)} \mathbb{1}\left(\widetilde{S}^{W}\left(w^{\prime \prime}, \mathbf{V}^{\prime \prime}\right)<\varepsilon^{W \prime \prime} \vee \widetilde{S}^{F}\left(w^{\prime \prime}, \mathbf{V}^{\prime \prime}\right)<\varepsilon^{F \prime}\right) K\left(\left(w^{\prime \prime}, \mathbf{V}^{\prime \prime}\right) \mid\left(w^{\prime}, \mathbf{V}^{\prime}\right)\right) d\left(w^{\prime \prime}, \mathbf{V}^{\prime \prime}\right)}_{\equiv \widetilde{\mathbb{D}}\left(w^{\prime}, \mathbf{V}^{\prime} ; \varepsilon^{W \prime}, \varepsilon^{F \prime}\right)} \\
& f_{\text {post }}^{Z}\left(w^{\prime}, \mathbf{V}^{\prime}\right) d\left(w^{\prime}, \mathbf{V}^{\prime}\right) .
\end{aligned}
$$

The non-Coasean analogue of the Coasean predicted post-REBP separation rate 17) is (from a closely analogous derivation):

$$
\Delta^{1}=\frac{1-\delta^{0}}{1-\delta^{1}}\left[\Delta^{0}-\int_{\left(w^{\prime}, \mathbf{V}^{\prime}\right) \in M^{\prime N C}} \widetilde{\mathbb{D}}\left(w^{\prime}, \mathbf{V}^{\prime} ; \varepsilon^{W \prime \prime}, \varepsilon^{F \prime \prime}\right) f_{\text {post }}^{0}\left(w^{\prime}, \mathbf{V}^{\prime}\right) d\left(w^{\prime}, \mathbf{V}^{\prime}\right)\right]
$$

As in the Coasean case, the post-REBP separation behavior of the former treatment group tracks that of the former control group, except for the contribution of the marginal jobs $\left(\left(w^{\prime}, \mathbf{V}^{\prime}\right) \in M^{\prime N C}\right)$ to such separation behavior. Unlike in the Coasean setting, these missing matches are marginal with respect to worker surplus - the dimension along which REBP selects them into separation but not necessarily with respect to a firm surplus shock.

The Incidence of Worker vs. Firm Surplus Shifts In fact, this non-Coasean model can rationalize the observed patterns of separations even if we assume perfect persistence in job surplus following the REBP abolition (an assumption perhaps particularly plausible within the one-year interval following the abolition to 1995). By contrast, the Coasean model was not able to explain the empirical post-REBP separation behavior, except if one were willing to assume full reshuffling in idiosyncratic surplus.

Figure 10 Panels (c) and (d) plot post-REBP separations in the former treatment and control groups as a function of post-REBP surplus shocks (for the perfect persistence case). It illustrates that the initial incidence of a given surplus shock - on the firm or worker side - matters: for worker shocks, the line representing post-REBP separations in the formerly treated group is flat up until

\footnotetext{
${ }^{50}$ Formally in terms of densities, after REBP is abolished, the former treatment group's post-REBP surplus distribution $f_{\text {post }}^{1}($.$) is now again truncated, but specifically with regards to worker rather than joint surplus:$

$f_{\text {post }}^{1}\left(w^{\prime}, \mathbf{V}^{\prime}\right)=\left\{\begin{array}{cl}0 & \text { if } \quad\left(w^{\prime}, \mathbf{V}^{\prime}\right) \notin\left(J^{\prime N C} \backslash M^{\prime N C}\right) \Leftrightarrow \widetilde{S}^{W}\left(w^{\prime}, \mathbf{V}^{W \prime}\right)<\varepsilon_{b}^{W \prime} \vee \widetilde{S}^{F}\left(w^{\prime}, \mathbf{V}^{F \prime}\right)<0 \\ \frac{f_{\text {post }}^{0}\left(w^{\prime}, \mathbf{V}^{\prime}\right)}{1-\int_{\left(w^{\prime}, \mathbf{V}^{\prime}\right) \in M^{\prime} N C} f_{\text {post }}^{0}\left(w^{\prime}, \mathbf{V}^{\prime}\right) d\left(w^{\prime}, \mathbf{V}^{\prime}\right)} & \text { if } \quad\left(w^{\prime}, \mathbf{V}^{\prime}\right) \in\left(J^{N C} \backslash M^{\prime N C}\right) \Leftrightarrow \widetilde{S}^{W}\left(w^{\prime}, \mathbf{V}^{W \prime}\right) \geq \varepsilon_{b}^{W \prime} \wedge \widetilde{S}^{F}\left(w^{\prime}, \mathbf{V}^{F \prime}\right) \geq 0 .\end{array}\right.$
} 
the point hits the truncation point of surplus, and then increases somewhat more steeply. For firm shocks however, the separation rates can be very similar between the former treamtent and control groups in our example, as also depicted in the case study Figure 11 Panels (b.NC) and (c.NC). This pattern differs from the Coasean setting, where only joint surplus and hence the net sum of shocks matters, and resilience was unambiguously predicted (provided some persistence). Below we derive these cases.

As in the Coasean case, our objective is to rewrite expression (26) in an empirically tractable form of realized control group separation rates (and the original size of the REBP treatment effects). In order to do this, we first specify the model to feature persistence of idiosyncratic job surplus (while still permitting any structure on $k\left(\left(w^{\prime}, \mathbf{V}^{\prime}\right) \mid(w, \mathbf{V})\right)$ i.e. no restriction on idiosyncratic shocks during the five years REBP was active), such that $K\left(\left(w^{\prime \prime}, \mathbf{V}^{\prime \prime}\right) \mid\left(w^{\prime}, \mathbf{V}^{\prime}\right)\right)=1$ if $\left(w^{\prime \prime}, \mathbf{V}^{\prime \prime}\right)=\left(w^{\prime}, \mathbf{V}^{\prime}\right)$ and 0 otherwise, such that for $Z=0,1$, post-REBP separation rates given by (25) are specified to:

$$
\Delta^{Z}=\int_{\left(w^{\prime}, \mathbf{V}^{\prime}\right)} \mathbb{1}\left(\widetilde{S}^{W}\left(w^{\prime}, \mathbf{V}^{W \prime}\right)<\varepsilon^{W^{\prime \prime}} \vee \widetilde{S}^{F}\left(w^{\prime}, \mathbf{V}^{F \prime}\right)<\varepsilon^{F^{\prime \prime}}\right) f_{\text {post }}^{Z}\left(w^{\prime}, \mathbf{V}^{\prime}\right) d\left(w^{\prime}, \mathbf{V}^{\prime}\right)
$$

Then, the general relationship between treatment and control separations given by Equation (26) is specified to:

$$
\Delta^{1}=\frac{1-\delta^{0}}{1-\delta^{1}}\left[\Delta^{0}-\int_{\left(w^{\prime}, \mathbf{V}^{\prime}\right) \in M^{\prime N C}} \mathbb{1}\left(\widetilde{S}^{W}\left(w^{\prime}, \mathbf{V}^{W \prime}\right)<\varepsilon^{W^{\prime \prime}} \vee \widetilde{S}^{F}\left(w^{\prime}, \mathbf{V}^{F \prime}\right)<\varepsilon^{F^{\prime \prime}}\right) f_{\text {post }}^{0}\left(w^{\prime}, \mathbf{V}^{\prime}\right) d\left(w^{\prime}, \mathbf{V}^{\prime}\right)\right] .
$$

As a second and last step to obtaining empirically tractable expressions, we now distinguish two cases: only worker or only firm shocks driving separations.

Resilience: Post-REBP Separations Driven by Worker Surplus First, suppose most (all) postREBP separations arise from worker shocks. In this case, the formerly treated group again exhibits resilience in form of a piece-wise linear comovement between treatment and control separations featuring a flat-at-zero region, mirroring the Coasean case. This analogue arises because the selection during REBP was with respect to the same allocative concept post-REBP. Accordingly, the resulting expression is analogous to Coasean Equation (18):51

\footnotetext{
${ }^{51}$ To see this, consider again the two cases, $\varepsilon^{W \prime \prime} \leq \varepsilon_{b}^{W \prime}$ and $\varepsilon^{W \prime \prime}>\varepsilon_{b}^{W \prime}$, in order to derive $\Delta_{\varepsilon^{W \prime \prime}>\varepsilon_{b}^{W}}^{1}\left(\varepsilon^{W \prime \prime}\right)$ and $\Delta_{\varepsilon^{W \prime \prime} \leq \varepsilon_{b}^{W}}^{1}\left(\varepsilon^{W \prime \prime}\right)$ similarly to the Coasean persistence case. For the case of $\varepsilon^{W \prime \prime} \leq \varepsilon_{b}^{W \prime}$, it holds that jobs for which $\widetilde{S}^{W}\left(w^{\prime}, \mathbf{V}^{W \prime}\right)<\varepsilon^{W^{\prime \prime}}$ also have $\widetilde{S}^{W}\left(w^{\prime}, \mathbf{V}^{W \prime}\right)<\varepsilon_{b}^{W^{\prime}}$ and hence these jobs were in the marginal set w.r.t. $\operatorname{REBP}\left(w^{\prime}, \mathbf{V}^{\prime}\right) \in$ $M^{\prime N C}$. Therefore, using Equation 27, we have for the case of $\varepsilon^{W \prime \prime} \leq \varepsilon_{b}^{W \prime}$ (i.e. now we can limiting the integral to $\left.M^{\prime N C}\right)$ :

$$
\Delta^{0}\left(\varepsilon^{W \prime \prime}\right)=\int_{\left(w^{\prime}, \mathbf{V}^{\prime}\right) \in M^{\prime N C}} \mathbb{1}\left(\widetilde{S}^{W}\left(w^{\prime}, \mathbf{V}^{W \prime}\right)<\varepsilon^{W^{\prime \prime}} \vee \widetilde{S}^{F}\left(w^{\prime}, \mathbf{V}^{F \prime}\right)<0\right) f_{\text {post }}^{0}\left(w^{\prime}, \mathbf{V}^{\prime}\right) d\left(w^{\prime}, \mathbf{V}^{\prime}\right)
$$




$$
\Delta^{1}\left(\Delta_{0}\left(\varepsilon^{W \prime \prime}\right), \delta_{0}, \delta_{1}\right)=\max \left\{0, \frac{1-\delta^{0}}{1-\delta^{1}}\left[\Delta^{0}\left(\varepsilon^{W \prime \prime}\right)-\frac{\delta^{1}-\delta^{0}}{1-\delta^{0}}\right]\right\} .
$$

Figure 10 Panel (b) plots Equation (31), post-REBP treatment group against control group separations for this case (red dashed line).

Therefore, when there is perfect persistence and only worker shocks, the marginal jobs are those which separate first and hence the average separation rate of the inframarginal jobs is lower than that of the marginal jobs, resulting in $\Delta^{1}\left(\varepsilon^{W \prime \prime}\right) \leq \Delta^{0}\left(\varepsilon^{W \prime \prime}\right)$ as in Equation (31).

Perfect Comovement: Post-REBP Separations Driven by Firm Surplus We now ask which properties let the non-Coasean model rationalize the (empirically consistent) comovement between the groups post-abolition. Of course, making an assumption of perfect reshuffling right after the abolition could again generate the perfect comovement in the non-Coasean setting (which was the only way the Coasean setting could rationalize this pattern) 52 Yet, additionally even with persistence in idiosyncratic surplus, the non-Coasean model can rationalize very similar separation sensitivities between the treatment and control group REBP survivors: if post-REBP separations are largely due to firm surplus shocks. More precisely and subtly, another ingredient is that worker and firm surplus are approximately independently distributed, since REBP extracted jobs that were marginal with respect to worker (rather than firm) surplus.

With firm shocks, again assuming persistence in job surplus right after REBP is abolished but permitting arbitrary surplus evolution during REBP, the empirical relationship between postREBP separation rates in the treatment and in the control group is driven by the relative separation behavior of marginal and inframarginal matches with respect to firm shocks - which in turn is determined by the distribution of firm surplus in the marginal vs. inframarginal matches. To formally derive this result, we start from the general relationship between the separation rates in

which implies that $\Delta_{\varepsilon^{W \prime \prime}>\varepsilon_{b}^{W}}^{1},\left(\varepsilon^{W \prime \prime}\right)=0$ by Equation 28. By contrast, for the case of $\varepsilon^{W \prime \prime}>\varepsilon_{b}^{W \prime}$, all marginal-to-REBP jobs $\left(w^{\prime}, \mathbf{V}^{\prime}\right) \in M^{\prime N C}$ satisfy the condition $\left(\widetilde{S}^{W}\left(w^{\prime}, \mathbf{V}^{W \prime}\right)<\varepsilon^{W^{\prime \prime}} \vee \widetilde{S}^{F}\left(w^{\prime}, \mathbf{V}^{F \prime}\right)<0\right)$, and therefore Equation 28 becomes

$$
\begin{aligned}
\Delta_{\varepsilon^{W \prime} \leq \varepsilon_{b}^{W}}^{1}\left(\varepsilon^{W \prime \prime}\right) & =\frac{1-\delta^{0}}{1-\delta^{1}}\left[\Delta^{0}\left(\varepsilon^{W \prime \prime}\right)-\int_{\left(w^{\prime}, \mathbf{V}^{\prime}\right) \in M^{\prime N C}} \mathbb{1}\left(\widetilde{S}^{W}\left(w^{\prime}, \mathbf{V}^{W \prime}\right)<\varepsilon^{W^{\prime \prime}} \vee \widetilde{S}^{F}\left(w^{\prime}, \mathbf{V}^{F \prime}\right)<0\right) f_{\text {post }}^{0}\left(w^{\prime}, \mathbf{V}^{\prime}\right) d\left(w^{\prime}, \mathbf{V}^{\prime}\right)\right] \\
& =\frac{1-\delta^{0}}{1-\delta^{1}}\left[\Delta^{0}\left(\varepsilon^{W \prime \prime}\right)-\int_{\left(w^{\prime}, \mathbf{V}^{\prime}\right) \in M^{\prime N C}} f_{\text {post }}^{0}\left(w^{\prime}, \mathbf{V}^{\prime}\right) d\left(w^{\prime}, \mathbf{V}^{\prime}\right)\right] \\
& =\frac{1-\delta^{0}}{1-\delta^{1}}\left[\Delta^{0}\left(\varepsilon^{W \prime \prime}\right)-\frac{\delta^{1}-\delta^{0}}{1-\delta^{0}}\right]
\end{aligned}
$$

where $\frac{\delta^{1}-\delta^{0}}{1-\delta^{0}}$ is the fraction of marginal jobs in the control group. Combining the two cases, we obtain Equation (31).

${ }^{52}$ Perfect comovement requires that, in response to the shock being considered, the average separation rate of the marginal jobs is equal to the average separation rate of the inframarginal jobs. The same identical derivation as in Section 5.3, paragraph “Case II: Perfect Reshuffling” applies: simply replace $\mathbf{V}^{\prime}$ with $\left(w^{\prime}, \mathbf{V}^{\prime}\right), \widetilde{\mathbb{D}}\left(\mathbf{V}^{\prime}, \varepsilon^{\prime \prime}\right)$ with $\widetilde{\mathbb{D}}\left(w^{\prime}, \mathbf{V}^{\prime} ; \varepsilon^{W \prime \prime}, \varepsilon^{F \prime \prime}\right), M^{\prime}$ and $J^{\prime}$ with $M^{\prime N C}$ and $J^{\prime N C}$ respectively. This condition holds for any shock and any joint density $f_{\text {post }}^{0}\left(w^{\prime} ; \mathbf{V}^{W^{\prime}}, \mathbf{V}^{F^{\prime}}\right)$ when there is perfect reshuffling in surplus. Additionally, it holds for only-firm-shocks when there is perfect persistence and firm and worker surpluses are independently distributed, as discussed below. 
the non-Coasean setting (with two unilateral surpluses and participation constraints) $\sqrt[53]{5}$

$$
\begin{aligned}
& \Delta^{1}\left(\varepsilon^{F \prime \prime}, \delta^{0}, \delta^{1}\right) \lesseqgtr \Delta^{0}\left(\varepsilon^{F \prime \prime}, \delta^{0}, \delta^{1}\right) \\
& \Leftrightarrow \int_{\left(w^{\prime}, \mathbf{V}^{\prime}\right) \in\left(J^{\prime N C} \backslash M^{\prime N C}\right)} \mathbb{1}\left(\widetilde{S}^{W}\left(w^{\prime}, \mathbf{V}^{W \prime}\right)<0 \vee \widetilde{S}^{F}\left(w^{\prime}, \mathbf{V}^{F \prime}\right)<\varepsilon^{F^{\prime \prime}}\right) \tilde{f}_{I}^{0}\left(w^{\prime}, \mathbf{V}^{\prime}\right) d\left(w^{\prime}, \mathbf{V}^{\prime}\right) \\
& \lesseqgtr \int_{\left(w^{\prime}, \mathbf{V}^{\prime}\right) \in M^{\prime N C}} \mathbb{1}\left(\widetilde{S}^{W}\left(w^{\prime}, \mathbf{V}^{W \prime}\right)<0 \vee \widetilde{S}^{F}\left(w^{\prime}, \mathbf{V}^{F \prime}\right)<\varepsilon^{F^{\prime \prime}}\right) \tilde{f}_{M}^{0}\left(w^{\prime}, \mathbf{V}^{\prime}\right) d\left(w^{\prime}, \mathbf{V}^{\prime}\right) \\
& \Leftrightarrow \int_{\left(w^{\prime}, \mathbf{V}^{\prime}\right) \in\left(J^{\prime N C} \backslash M^{\prime N C}\right)} \mathbb{1}\left(\widetilde{S}^{F}\left(w^{\prime}, \mathbf{V}^{F \prime}\right)<\varepsilon^{F^{\prime \prime}}\right) \tilde{f}_{I}^{0}\left(w^{\prime}, \mathbf{V}^{\prime}\right) d\left(w^{\prime}, \mathbf{V}^{\prime}\right) \\
& \lesseqgtr \int_{\left(w^{\prime}, \mathbf{V}^{\prime}\right) \in M^{\prime N C}} \mathbb{1}\left(\widetilde{S}^{F}\left(w^{\prime}, \mathbf{V}^{F \prime}\right)<\varepsilon^{F^{\prime \prime}}\right) \tilde{f}_{M}^{0}\left(w^{\prime}, \mathbf{V}^{\prime}\right) d\left(w^{\prime}, \mathbf{V}^{\prime}\right) \\
& \Leftrightarrow \operatorname{Prob}\left(0 \leq \widetilde{S}^{F}\left(w^{\prime}, \mathbf{V}^{F \prime}\right)<\varepsilon^{F^{\prime \prime}} \mid \widetilde{S}^{W}\left(w^{\prime}, \mathbf{V}^{W \prime}\right) \geq \varepsilon_{b}^{W \prime}\right) \\
& \lesseqgtr \operatorname{Prob}\left(0 \leq \widetilde{S}^{F}\left(w^{\prime}, \mathbf{V}^{F \prime}\right)<\varepsilon^{F^{\prime \prime}} \mid 0 \leq \widetilde{S}^{W}\left(w^{\prime}, \mathbf{V}^{W \prime}\right)<\varepsilon_{b}^{W \prime}\right) \text {, }
\end{aligned}
$$

where $\tilde{f}_{I}^{0}\left(w^{\prime}, \mathbf{V}^{\prime}\right)=f_{\text {post }}^{0}\left(w^{\prime}, \mathbf{V}^{\prime}\right)\left[\frac{1-\delta^{0}}{1-\delta^{1}}\right]$ is the density of the inframarginal jobs in the control group and $\tilde{f}_{M}^{0}\left(w^{\prime}, \mathbf{V}^{\prime}\right)=f_{\text {post }}^{0}\left(w^{\prime}, \mathbf{V}^{\prime}\right)\left[\frac{1-\delta^{0}}{\delta^{1}-\delta^{0}}\right]$ is the density of the marginal jobs in the control group.

The second step recognizes that condition $\widetilde{S}^{W}\left(w^{\prime}, \mathbf{V}^{W \prime}\right)<0$ is slack without worker aggregate shocks and with persistence in idiosyncratic surplus (i.e. for these jobs, $\left.f_{\text {post }}^{0}\left(w^{\prime}, \mathbf{V}^{W^{\prime}}, \mathbf{V}^{F \prime}\right)=0\right)$.

The third step clarifies that the conditions now compare two simple conditional cumulative distribution functions of firm surplus with threshold given by the firm surplus shock, for the range of worker surplus shocks partitioned by the REBP surplus cutoff ${ }^{54}$

${ }^{53}$ This expression is derived by specializing Equation (27) to the case of firm shocks only, and then, analogously to the Coasean derivation [19], combining the inframarginal jobs in the treatment and control groups on one side, using:

$$
\begin{aligned}
\Delta^{1}\left(\varepsilon^{F \prime \prime}\right) & =\int_{\left(w^{\prime}, \mathbf{V}^{\prime}\right)} \mathbb{1}\left(\widetilde{S}^{W}\left(w^{\prime}, \mathbf{V}^{W \prime}\right)<0 \vee \widetilde{S}^{F}\left(w^{\prime}, \mathbf{V}^{F \prime}\right)<\varepsilon^{F^{\prime \prime}}\right) f_{\text {post }}^{1}\left(w^{\prime}, \mathbf{V}^{\prime}\right) d\left(w^{\prime}, \mathbf{V}^{\prime}\right) \\
& =\int_{\left(w^{\prime}, \mathbf{V}^{\prime}\right) \in\left(J^{\prime N C} \backslash M^{\prime N C}\right)} \mathbb{1}\left(\widetilde{S}^{W}\left(w^{\prime}, \mathbf{V}^{W \prime}\right)<0 \vee \widetilde{S}^{F}\left(w^{\prime}, \mathbf{V}^{F \prime}\right)<\varepsilon^{F^{\prime \prime}}\right) f_{\text {post }}^{0}\left(w^{\prime}, \mathbf{V}^{\prime}\right)\left[\frac{1-\delta^{0}}{1-\delta^{1}}\right] d\left(w^{\prime}, \mathbf{V}^{\prime}\right),
\end{aligned}
$$

where the second equality follows from Equation [24] (after reformulating the densities there into $1-\delta^{0}$ and $1-\delta^{1}$ ).

${ }^{54} \mathrm{To}$ see this formally, note that the property of joint densities implies that $f_{\text {post }}^{0}\left(w^{\prime}, \mathbf{V}^{W \prime}, \mathbf{V}^{F^{\prime}}\right)=$ $f_{\text {post }}^{0}\left(w^{\prime}, \mathbf{V}^{F \prime} \mid w^{\prime}, \mathbf{V}^{W \prime}\right) f_{\text {post }}^{0}\left(w^{\prime}, \mathbf{V}^{W \prime}\right)$, we can write condition 34 as

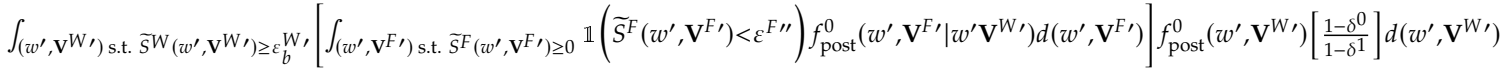

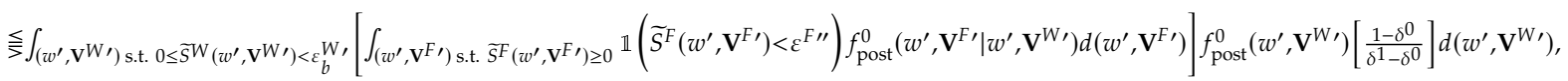

which we can rewrite in terms of surpluses directly, defining densities of surpluses $h($.) rather than of job/wage attributes 
Our empirically interesting case is:

$$
\Delta^{1}\left(\varepsilon^{F \prime \prime}, \delta^{0}, \delta^{1}\right)=\Delta^{0}\left(\varepsilon^{F \prime \prime}, \delta^{0}, \delta^{1}\right),
$$

such that the two post-abolition separation rates are equal for all post-REBP firm shocks and moreover for any size of the set of marginal jobs REBP extracted $\left(\delta^{1}-\delta^{0}\right)$. This "global" condition is fulfilled if worker and firm surpluses are independently distributed 55 The non-Coasean setting can then rationalize our findings of no post-REBP resilience whatsoever even with perfect persistence in idiosyncratic job surplus.

We show below that we can now conveniently reinterpret the mixed model within the nonCoasean framework, and then further discuss these ingredients for the non-Coasean framework to rationalize the full set of results.

\subsection{Reinterpreting the Structural Estimation of Mixed Model}

Together, Equations (31) and (36) permit us to again formulate a mixed model relating formerly treated jobs with control groups, as a weighted average of a 45 degree line (perfect comovement case in the firm surplus case) and the kinked piece-wise linear function (resilience in the worker surplus case):

$$
\begin{gathered}
\Delta_{i}^{1}=\kappa \times \underbrace{\Delta_{i}^{0}}_{\substack{\text { Coasean (or Non-Coasean) \& Reshuffling } \\
\text { OR }}} \\
\text { Non-Coasean \& Firm Shocks \& Persistence } \\
\text { Non-Coasean \& Worker Shocks \& Persistence }
\end{gathered}
$$

While in the data we cannot directly observe the incidence of shocks on the worker vs. firm side driving the separations, the estimated $\kappa$ of essentially one would then identify the share of separations due to firm-side shocks within the persistence framework.

$\overline{f_{\text {post }}(.) \text { : }}$

$$
\begin{aligned}
& \int_{\widetilde{S}^{W^{\prime}} \geq \varepsilon_{b}^{W,}}\left[\int_{\widetilde{S}^{F^{\prime}} \geq 0} \mathbb{1}\left(\widetilde{S}^{F}<\varepsilon^{F \prime \prime}\right) h_{\text {post }}^{0}\left(\widetilde{S}^{F^{\prime}} \mid \widetilde{S}^{W \prime}\right) d \widetilde{S}^{F^{\prime}}\right] h_{\text {post }}^{0}\left(\widetilde{S}^{W \prime}\right)\left[\frac{1-\delta^{0}}{1-\delta^{1}}\right] \widetilde{S}^{W \prime} \\
& \lesseqgtr \int_{0 \leq \widetilde{S}^{W^{\prime}}<\varepsilon_{b}^{W \prime}}\left[\int_{\widetilde{S}^{F^{\prime}} \geq 0} \mathbb{1}\left(\widetilde{S}^{F \prime}<\varepsilon^{F \prime \prime}\right) h_{\text {post }}^{0}\left(\widetilde{S}^{F \prime} \mid \widetilde{S}^{W \prime}\right) d \widetilde{S}^{F \prime}\right] h_{\text {post }}^{0}\left(\widetilde{S}^{W \prime}\right)\left[\frac{1-\delta^{0}}{\delta^{1}-\delta^{0}}\right] d \widetilde{S}^{W \prime} \text {. }
\end{aligned}
$$

Up until now we rewrote condition [34. Now assume that worker surplus and firm surpluses are independent: $h_{\text {post }}^{0}\left(\widetilde{S}^{F^{\prime}} \mid \widetilde{S}^{W \prime}\right)=h_{\text {post }}^{0}\left(\widetilde{S}^{F \prime}\right)$. Then condition 34 collapses to equality. That is, if worker and firm surpluses are independently distributed, $\Delta^{1}\left(\varepsilon^{F \prime}, \delta^{0}, \delta^{1}\right)=\Delta^{0}\left(\varepsilon^{F \prime}, \delta^{0}, \delta^{1}\right)$, i.e. post-REBP separation rates co-move perfectly in response to a firm shock even if there is perfect persistence of surplus for one period to the next.

${ }^{55} \mathrm{Of}$ course, in practice, the shocks may be percent shifters of the given job surplus, so that the condition would not literally need to apply in levels. 


\subsection{Discussion of the Non-Coasean Framework}

We now discuss the implied non-Coasean constellation, specifically high worker surplus and low firm surplus, with limited correlation between the two, by which the non-Coasean setting rationalizes the empirical findings. We also discuss the concrete real-world sources potentially generating these conditions.

Initially High Worker Surplus and Low Firm Surplus Strictly interpreted, our firm-shock-only setting assumes that no aggregate worker shocks hit. A more realistic interpretation is that worker shocks are either smaller than firm shocks, or worker shocks are similarly sized and frequent. But, worker surplus is initially high in our sample. This would let REBP - a massive policy shift - extract some marginal matches that otherwise (as in the control group) would have remained inframarginal.

High worker surplus is particularly plausible for our sample - older, and high-tenured workers - and that exact constellation turns out to be predicted by the long-standing hypotheses of implicit contract models, in form of backloading of compensation over the job spell (Lazear (1979, 1981)): in a period-by-period consideration, young workers are "underpaid", while older workers are "overpaid." This backloading is supported by implicit contracts or formal institutions. 56 Perhaps additionally supporting such implicit contracts (and also limiting the selectivity of layoffs, and perhaps overall in line with the findings in Bewley, 2002) is the Austrian institutional setting, which features an explicit consultation role for works councils in the separation process.

Lastly, Austria mandates multiple months of severance payments in the case of layoffs that are foregone for quitters, providing little incentive for workers to quit unilaterally (despite full UI eligibility for quitters in Austria, as discussed in Section 2).

Large Worker Surplus Shift From REBP For the above scenario, when worker surplus is initially high, the boosts to worker outside option must be large to sweep up otherwise inframarginal workers. The exceptional size of the initial UI treatment - four years of full UI eligibility, hence also serving as a bridge into early retirement - achieved this. In Appendix Section A, we benchmark that, for the average worker, the cash value corresponds to $71 \%$ of annual earnings: ${ }^{57}$

Limited Correlation Between Initial Firm and Worker Surpluses In the non-Coasean setting, the low-worker surplus jobs REBP extracted evidently were not marginal with respect to firm surplus. Such limited correlation can arise if firm and worker gross-of-wage fundamentals $\mathbf{V}^{F}$ and $\mathbf{V}^{W}$ are uncorrelated, or if net-of-wage surpluses are in an extreme case independently distributed, for example due to wage frictions. (By contrast, in the Coasean setting, the correlation between

${ }^{56}$ While models of job ladders and negotiation capital such as Cahuc et al. (2006) generate this joint distribution, these models feature bilaterally efficient (re-)bargaining and separations.

${ }^{57} \mathrm{~A}$ cross-validation test is that smaller shifts in UI should not induce workers to separate. Jäger et al. (2018), which document that wages are insulated from UI shifts, find that smaller shifts in the UI benefit level and smaller duration extensions did not entail separation effects even among older workers and similar time periods in Austria. 
initial worker and firm surpluses is irrelevant due to rebargaining.) 58 Ex post, wage patterns that may limit the correlation of the two unilateral surpluses may in fact emerge in certain contracting models (Harris and Holmstrom, 1982: McLaughlin, 1991; MacLeod and Malcomson, 1993) due to initially set wages that then need not evolve with idiosyncratic productivity. In our model, it is crucial that the wages are rigid and allocative at the separation margin rather than renegotiated. In the data in Section 4, we have found evidence consistent with firm surplus - which drives post-REBP separations - not being correlated with the REBP selection along worker surplus, as the labor demand shocks indeed did not affect separations less among REBP cohorts. Moreover, our complier analysis in Section 3.2 revealed that wages did not differ systematically between marginal jobs and inframarginal jobs.

\section{Conclusion}

Labor market quantities adjust largely along the employment (extensive) margin rather than the hours (intensive) margin. At the micro level, such extensive-margin adjustments play out as discrete choice problems within employment relationships of individual workers and firms that are destroyed, maintained or formed, with a basic condition: each party must be better off inside the job than with her respective outside option, hence enjoying surplus from the job. By this theory, the distribution of heterogeneous job surpluses determines the level of employment, and its adjustment (Mortensen and Pissarides, 1994; Bils et al., 2012: Mui and Schoefer, 2019).

An open question is which theories of job formation and separation guide employment adjustment. According to the popular Coasean approach, the parties exploit all gains from trade by using arbitrarily complicated and abstract contractual arrangements to transfer utility. On the basis of observed separation behavior, this Coasean view is difficult to empirically distinguish from non-Coasean alternatives, in which wage or other frictions and institutional constraints permit inefficient separations.

We empirically adjudicate between the Coasean and non-Coasean view by tracking jobs longitudinally over the course of the introduction and sudden abolition of a policy that subsidized nonemployment, hence lowered job surplus, and should have entailed separations: an age-andregion-specific extension of unemployment insurance duration from one to four years in Austria. The Coasean view would predict resilience in the post-abolition separation behavior. By contrast, the inefficient separations in non-Coasean models can accommodate that after the abolition, the survivors in the treatment and control groups move in lock step - exactly in line with the data, as

\footnotetext{
${ }^{58}$ In fact, the non-Coasean model could even rationalize higher separations among the former treatment group in response to firm shocks under a "random" wage triggering a negative correlation between worker and firm surplus: REBP quitters would then be very underpaid - and hence particularly valuable to firms, and REBP would have removed low worker surplus jobs but also high firm surplus jobs. Some of our evidence may suggest such short-run "antiresilience". Appendix Figure A.5 documents additional separations in the formerly treated group between $1993 \mathrm{q} 3$ to $1994 \mathrm{q} 1$ for the bottom quintile of industry growth cells. Figure 9 documents slightly more sensitive slopes in the "hockey stick" for formerly treated cohorts. However, any such short run "phantom treatment effects" may arise from the institutional grandfathering of pre-scheduled layoffs due to advance notifications as required by law, of multiple months.
} 
we document.

Our revealed-preference test suggests an empirical rejection of the Coasean hypothesis, at least in the context of the Austrian labor market among workers in their 50s in the late 1980s and early 1990s, in response to a UI reform that boosted workers' nonemployment outside option. We present and discuss one specific non-Coasean mechanism, building on wage rigidity, that can rationalize the findings, although we can not definitely pinpoint the specific sources of the inefficiencies. Moreover, our specific case study must naturally leave for future research the external validity of our findings, which is of substantive interest given the welfare consequences: whether, and which, other institutional or demographic contexts can come closer to complying with the Coasean benchmark of efficient separations. Our revealed preference test may offer an empirical handle on how to adjudicate between Coasean and non-Coasean separation mechanisms in such contexts. 


\section{References}

Abadie, A. (2003). Semiparametric Instrumental Variable Estimation of Treatment Response Models. Journal of Econometrics 113(2), 231-263.

Abowd, J. M., F. Kramarz, and D. N. Margolis (1999). High Wage Workers and High Wage Firms. Econometrica 67(2), 251-333.

Akerlof, G. A. and J. L. Yellen (1986). Efficiency Wage Models of the Labor Market. Cambridge University Press.

Angrist, J. D. and I. Fernandez-Val (2013). ExtrapoLATE-ing: External Validity and Overidentification in the LATE Framework. In Advances in Economics and Econometrics: Volume 3, Econometrics: Tenth World Congress, Volume 51, pp. 401. Cambridge University Press.

Angrist, J. D. and J.-S. Pischke (2008). Mostly Harmless Econometrics: An Empiricist's Companion. Princeton University Press.

Bagger, J., F. Fontaine, F. Postel-Vinay, and J.-M. Robin (2014). Tenure, Experience, Human Capital, and Wages: A Tractable Equilibrium Search Model of Wage Dynamics. The American Economic Review 104(6), 1551-96.

Barro, R. J. (1977). Long-Term Contracting, Sticky Prices, and Monetary Policy. Journal of Monetary Economics 3(3), 305-316.

Basu, R. (2009). High Ambient Temperature and Mortality: A Review of Epidemiologic Studies from 2001 to 2008. Environmental Health 8(1), 40.

Beaudry, P., D. A. Green, and B. Sand (2012). Does Industrial Composition Matter for Wages? A Test of Search and Bargaining Theory. Econometrica 80(3), 1063-1104.

Bewley, T. F. (2002). Why Wages Don't Fall during a Recession. Harvard University Press.

Bils, M., Y. Chang, and S.-B. Kim (2012). Comparative Advantage and Unemployment. Journal of Monetary Economics 59(2), 150-165.

Cahuc, P., F. Postel-Vinay, and J.-M. Robin (2006). Wage Bargaining with On-the-Job Search: Theory and Evidence. Econometrica 74(2), 323-64.

Card, D., A. R. Cardoso, J. Heining, and P. Kline (2018). Firms and Labor Market Inequality: Evidence and Some Theory. Journal of Labor Economics 36(S1), S13-S70.

Card, D., R. Chetty, and A. Weber (2007). Cash-on-Hand and Competing Models of Intertemporal Behavior: New Evidence from the Labor Market. The Quarterly Journal of Economics 122(4), 1511-1560. 
Correia, S. et al. (2016). REGHDFE: Stata Module to Perform Linear or Instrumental-Variable Regression Absorbing any Number of High-Dimensional Fixed Effects. Statistical Software Components.

Davis, S. J., R. J. Faberman, and J. C. Haltiwanger (2013). The Establishment-Level Behavior of Vacancies and Hiring. The Quarterly Journal of Economics 128(2), 581-622.

De Chaisemartin, C. and X. D'Haultfoeuille (2018). Fuzzy Differences-in-Differences. The Review of Economic Studies 85(2), 999-1028.

Doornik, B., D. Schoenherr, and J. Skrastins (2018). Unemployment Insurance, Strategic Unemployment, and Firm-Worker Collusion. Working Paper.

Feldstein, M. (1976). Temporary Layoffs in the Theory of Unemployment. Journal of Political Economy 84(5), 937-957.

Fink, M., E. Kalkbrenner, A. Weber, and C. Zulehner (2010). Extracting Firm Information from Administrative Records: The ASSD Firm Panel. Austrian Center for Labor Economics and the Analysis of the Welfare State Working Paper No. 1004.

Frimmel, W., T. Horvath, M. Schnalzenberger, and R. Winter-Ebmer (2018). Seniority Wages and the Role of Firms in Retirement. Journal of Public Economics 164, 19-32.

Hagedorn, M. and I. Manovskii (2013). Job Selection and Wages over the Business Cycle. The American Economic Review 103(2), 771-803.

Hall, R. E. (2005). Employment Fluctuations with Equilibrium Wage Stickiness. The American Economic Review 95(1), 50-65.

Hall, R. E. and E. P. Lazear (1984). The Excess Sensitivity of Layoffs and Quits to Demand. Journal of Labor Economics 2(2), 233-257.

Hall, R. E. and P. R. Milgrom (2008). The Limited Influence of Unemployment on the Wage Bargain. The American Economic Review 98(4), 1653-74.

Hansen, G. D. (1985). Indivisible Labor and the Business Cycle. Journal of Monetary Economics 16(3), 309-327.

Harris, M. and B. Holmstrom (1982). A Theory of Wage Dynamics. The Review of Economic Studies 49(3), 315-333.

Hartung, B., P. Jung, and M. Kuhn (2018). What Hides behind the German Labor Market Miracle? Unemployment Insurance Reforms and Labor Market Dynamics. CEPR Discussion Paper No. DP13328.

Hudson, S., P. Hull, and J. Liebersohn (2017). Interpreting Instrumented Difference-in-Differences. Working Paper. 
Imbens, G. W. and D. B. Rubin (1997). Estimating Outcome Distributions for Compliers in Instrumental Variables Models. The Review of Economic Studies 64(4), 555-574.

Inderbitzin, L., S. Staubli, and J. Zweimüller (2016). Extended Unemployment Benefits and Early Retirement: Program Complementarity and Program Substitution. American Economic Journal: Economic Policy 8(1), 253-288.

Jäger, S. and J. Heining (2016). How Substitutable are Workers? Evidence from Worker Deaths. Working Paper.

Jäger, S. and B. Schoefer (2019). Labor in the Boardroom. Working Paper.

Jäger, S., B. Schoefer, S. Young, and J. Zweimüller (2018). Wages and the Value of Nonemployment. NBER Working Paper No. 25230.

Katz, L. F. (1986). Efficiency Wage Theories: A Partial Evaluation. NBER Macroeconomics Annual 1, 235-276.

Kyyrä, T. and R. A. Wilke (2007). Reduction in the Long-Term Unemployment of the Elderly: A Success Story from Finland. Journal of the European Economic Association 5(1), 154-182.

Lalive, R., C. Landais, and J. Zweimüller (2015). Market Externalities of Large Unemployment Insurance Extension Programs. The American Economic Review 105(12), 3564-3596.

Lalive, R., J. C. van Ours, and J. Zweimüller (2011). Equilibrium Unemployment and the Duration of Unemployment Benefits. Journal of Population Economics 24(4), 1385-1409.

Lazear, E. P. (1979). Why Is there Mandatory Retirement? Journal of Political Economy 87(6), 1261-1284.

Lazear, E. P. (1981). Agency, Earnings Profiles, Productivity, and Hours Restrictions. The American Economic Review 71(4), 606-620.

Li, T. (2002). Robust and Consistent Estimation of Nonlinear Errors-in-Variables Models. Journal of Econometrics 110(1), 1-26.

Li, T. and Q. Vuong (1998). Nonparametric Estimation of the Measurement Error Model Using Multiple Indicators. Journal of Multivariate Analysis 65(2), 139-165.

MacLeod, W. B. and J. M. Malcomson (1993). Investments, Holdup, and the Form of Market Contracts. The American Economic Review, 811-837.

McLaughlin, K. J. (1991). A Theory of Quits and Layoffs with Efficient Turnover. Journal of Political Economy 99(1), 1-29.

Mercan, Y. and B. Schoefer (forthcoming). Jobs and Matches: Quits, Replacement Hiring, and Vacancy Chains. American Economic Review: Insights. 
Mortensen, D. T. and C. A. Pissarides (1994). Job Creation and Job Destruction in the Theory of Unemployment. The Review of Economic Studies 61(3), 397-415.

Mui, P. and B. Schoefer (2019). The Aggregate Labor Supply Curve at the Extensive Margin: A Reservation Wedge Approach. Working Paper.

Nekoei, A. and A. Weber (2017). Does Extending Unemployment Benefits Improve Job Quality? The American Economic Review 107(2), 527-561.

OECD (1990). Economic Surveys: Austria 1988/1989. OECD Publishing.

Oi, W. Y. (1962). Labor as a Quasi-Fixed Factor. Journal of Political Economy 70(6), 538-555.

Pissarides, C. A. (2000). Equilibrium Unemployment Theory. MIT press.

Rogerson, R. (1988, January). Indivisible Labor, Lotteries and Equilibrium. Journal of Monetary Economics 21(1), 3-16.

Saez, E., B. Schoefer, and D. Seim (2019). Hysteresis from Employer Subsidies. Working Paper.

Schennach, S. M. (2004). Estimation of Nonlinear Models with Measurement Error. Econometrica $72(1), 33-75$.

Schennach, S. M. (2012). Measurement Error in Nonlinear Models: A Review. In Advances in Economics and Econometrics, Volume 3, Chapter 8. Econometric Society.

Schwartz, J. (2000). Harvesting and Long Term Exposure Effects in the Relation between Air Pollution and Mortality. American Journal of Epidemiology 151(5), 440-448.

Shapiro, C. and J. E. Stiglitz (1984). Equilibrium Unemployment as a Worker Discipline Device. The American Economic Review 74(3), 433-444.

Shimer, R. (2004). The Consequences of Rigid Wages in Search Models. Journal of the European Economic Association 2(2-3), 469-79.

Winter-Ebmer, R. (2003). Benefit Duration and Unemployment Entry: A Quasi-Experiment in Austria. European Economic Review 47(2), 259-273.

Zweimüller, J., R. Winter-Ebmer, R. Lalive, A. Kuhn, J.-P. Wuellrich, O. Ruf, and S. Buchi (2009). Austrian Social Security Satabase. Austrian Center for Labor Economics and the Analysis of the Welfare State Working Paper No. 0903. 


\section{Tables}

Table 1: Summary Statistics

\begin{tabular}{lcccc}
\hline \hline & \multicolumn{2}{c}{ 1988 Job Holders } & \multicolumn{2}{c}{ Age 50 Job Holders } \\
& Non-REBP Region & REBP Region & Non-REBP Region & REBP Region \\
& $(1)$ & $(2)$ & $(3)$ & $(4)$ \\
\hline \multirow{2}{*}{ Age } & 46.560 & 46.721 & 49.751 & 49.751 \\
& $(4.109)$ & $(4.145)$ & $(0.068)$ & $(0.068)$ \\
White Collar & 0.476 & 0.387 & 0.483 & 0.388 \\
& $(0.499)$ & $(0.487)$ & $(0.500)$ & $(0.487)$ \\
Experience & 20.103 & 21.420 & 20.436 & 21.781 \\
& $(6.353)$ & $(5.716)$ & $(6.848)$ & $(6.081)$ \\
Tenure & 9.605 & 10.640 & 10.516 & 10.720 \\
Annual Earnings (1,000 EUR) & $(5.974)$ & $(5.979)$ & $(7.187)$ & $(7.156)$ \\
& 36.285 & 36.137 & 37.633 & 37.966 \\
Log Annual Earnings & $(10.876)$ & $(10.044)$ & $(11.514)$ & $(10.525)$ \\
& 3.534 & 3.542 & 3.568 & 3.591 \\
Establishment Size & $(0.374)$ & $(0.326)$ & $(0.382)$ & $(0.324)$ \\
& 625.621 & 553.120 & 615.726 & 581.239 \\
Emp. Growth at Establishment & $(1684.252)$ & $(1037.453)$ & $(1674.651)$ & $(1107.786)$ \\
& 0.247 & 0.267 & 0.212 & 0.255 \\
Emp. Growth Industry & $(2.538)$ & $(4.202)$ & $(2.307)$ & $(4.302)$ \\
$\mathbb{1}$ (Growing Establishment) & 0.052 & 0.028 & 0.054 & 0.028 \\
& $(0.081)$ & $(0.078)$ & $(0.082)$ & $(0.077)$ \\
& 0.457 & 0.408 & 0.422 & 0.353 \\
& $(0.498)$ & $(0.491)$ & $(0.494)$ & $(0.478)$ \\
& & & & 86,387 \\
\hline
\end{tabular}

Note: The table reports results summary statistics for a sample of workers employed at the onset of the reform (1988q2) in columns (1) and (2) and for a sample of workers employed in the quarter before turning 50 in columns (3) and (4). "Non-REBP Region" and "REBP Region" refer to the labor market districts described in Section 2 and outlined in Panel (b) of Figure 1. excluding those districts that abolished REBP in 1991. Earnings are reported in (and log earnings are computed on) thousands of 2018 Euros. Details on the sample selection are in Section 2.3. 
Table 2: Difference-in-Differences Effects of the REBP on Outcomes of Initially Employed Workers (1988 to 1993)

\begin{tabular}{|c|c|c|c|c|c|}
\hline & $\begin{array}{c}(1) \\
\text { Separation }\end{array}$ & $\begin{array}{c}(2) \\
\text { Separation } \\
\text { Into Nonemployment }\end{array}$ & $\begin{array}{c}\text { (3) } \\
\text { Nonemployment } \\
\text { (Quarters) }\end{array}$ & $\begin{array}{c}(4) \\
\text { Unemp. (Benefits) } \\
\text { (Quarters) }\end{array}$ & $\begin{array}{c}\text { (5) } \\
\text { Cont. Empl. } \\
\text { (Quarters) }\end{array}$ \\
\hline REBP Region $\times$ Treated Cohort & $\begin{array}{c}0.109^{* * *} \\
(0.041)\end{array}$ & $\begin{array}{c}0.120^{* * *} \\
(0.043)\end{array}$ & $\begin{array}{c}1.461^{* * *} \\
(0.378)\end{array}$ & $\begin{array}{c}0.951^{*} \\
(0.531)\end{array}$ & $\begin{array}{c}-1.048^{* * *} \\
(0.365)\end{array}$ \\
\hline REBP Region & $\begin{array}{c}0.003 \\
(0.044)\end{array}$ & $\begin{array}{c}-0.003 \\
(0.008)\end{array}$ & $\begin{array}{c}-0.230 \\
(0.280)\end{array}$ & $\begin{array}{c}-0.101 \\
(0.182)\end{array}$ & $\begin{array}{c}0.024 \\
(0.677)\end{array}$ \\
\hline Treated Cohort & $\begin{array}{c}0.030 \\
(0.026)\end{array}$ & $\begin{array}{c}0.108^{* * *} \\
(0.005)\end{array}$ & $\begin{array}{c}0.805^{* * *} \\
(0.126)\end{array}$ & $\begin{array}{c}0.150^{* * *} \\
(0.056)\end{array}$ & $\begin{array}{c}0.146 \\
(0.391)\end{array}$ \\
\hline Constant & $\begin{array}{c}0.372^{* * *} \\
(0.098)\end{array}$ & $\begin{array}{c}0.057^{* * *} \\
(0.017)\end{array}$ & $\begin{array}{l}1.518^{* *} \\
(0.668)\end{array}$ & $\begin{array}{c}0.665 \\
(0.445)\end{array}$ & $\begin{array}{c}16.017^{* * *} \\
(1.820)\end{array}$ \\
\hline $\begin{array}{l}\text { Observations } \\
\text { Adjusted } R^{2} \\
\text { No of Clusters }\end{array}$ & $\begin{array}{c}390,791 \\
0.008 \\
100\end{array}$ & $\begin{array}{c}390,791 \\
0.046 \\
100\end{array}$ & $\begin{array}{c}390,791 \\
0.023 \\
100\end{array}$ & $\begin{array}{c}390,791 \\
0.018 \\
100\end{array}$ & $\begin{array}{c}390,791 \\
0.002 \\
100\end{array}$ \\
\hline
\end{tabular}

Note: The table reports results of the econometric specification in 11. REBP captures the effect of REBP-eligibility on the outcomes listed in columns (1) through (5) on a sample of workers employed at the onset of the reform (1988q2). We exclude workers born before 1933 and after 1948. Separation denotes an indicator function that is 1 if a worker separated from their 1988-employer by the end of the REBP period (1988q2 to 1993q3). Separation into Nonemployment denotes an indicator for Separation from the initial employer interacted with an indicator for not taking up employment with another employer. Nonemployment (Quarters), Unemployment (Benefits) (Quarters), and Continuous Employment (Quarters) denote the quarters of nonemployment, unemployment benefits, and continuous employment with the initial employer between 1988q2 and 1993q3. Standard errors clustered at the administrative region level are reported in parentheses. Levels of significance: * $10 \%$, ** $5 \%$, and ${ }^{* * *} 1 \%$. 
Table 3: Difference-in-Differences Effects of the REBP on Outcomes of Survivors (1994 Through 1996)

\begin{tabular}{|c|c|c|c|c|c|}
\hline & $\begin{array}{c}(1) \\
\text { Separation }\end{array}$ & $\begin{array}{c}(2) \\
\text { Separation } \\
\text { Into Nonemployment }\end{array}$ & $\begin{array}{c}\text { (3) } \\
\text { Nonemployment } \\
\text { (Quarters) }\end{array}$ & $\begin{array}{c}(4) \\
\text { Unemp. (Benefits) } \\
\text { (Quarters) }\end{array}$ & $\begin{array}{c}\text { (5) } \\
\text { Cont. Empl. } \\
\text { (Quarters) }\end{array}$ \\
\hline REBP Region $\times$ Treated Cohort & $\begin{array}{c}0.006 \\
(0.009)\end{array}$ & $\begin{array}{c}0.005 \\
(0.008)\end{array}$ & $\begin{array}{c}0.017 \\
(0.027)\end{array}$ & $\begin{array}{c}-0.072 \\
(0.045)\end{array}$ & $\begin{array}{r}-0.052 \\
(0.034)\end{array}$ \\
\hline REBP Region & $\begin{array}{c}-0.003 \\
(0.019)\end{array}$ & $\begin{array}{c}0.008 \\
(0.011)\end{array}$ & $\begin{array}{l}-0.007 \\
(0.056)\end{array}$ & $\begin{array}{c}0.005 \\
(0.041)\end{array}$ & $\begin{array}{c}0.116 \\
(0.088)\end{array}$ \\
\hline Treated Cohort & $\begin{array}{c}0.140^{* * *} \\
(0.009)\end{array}$ & $\begin{array}{c}0.164^{* * *} \\
(0.002)\end{array}$ & $\begin{array}{c}0.718^{* * *} \\
(0.010)\end{array}$ & $\begin{array}{l}0.145^{* *} \\
(0.069)\end{array}$ & $\begin{array}{c}-0.630^{* * *} \\
(0.057)\end{array}$ \\
\hline Constant & $\begin{array}{c}0.157^{* * *} \\
(0.051)\end{array}$ & $\begin{array}{l}0.068^{* *} \\
(0.030)\end{array}$ & $\begin{array}{l}0.324^{* *} \\
(0.142)\end{array}$ & $\begin{array}{c}0.136 \\
(0.107)\end{array}$ & $\begin{array}{l}8.166^{* * *} \\
(0.241)\end{array}$ \\
\hline $\begin{array}{l}\text { Observations } \\
\text { Adjusted } R^{2} \\
\text { No of Clusters }\end{array}$ & $\begin{array}{c}207,785 \\
0.026 \\
99\end{array}$ & $\begin{array}{c}207,785 \\
0.047 \\
99\end{array}$ & $\begin{array}{c}207,785 \\
0.038 \\
99\end{array}$ & $\begin{array}{c}207,785 \\
0.006 \\
99\end{array}$ & $\begin{array}{c}207,785 \\
0.018 \\
99\end{array}$ \\
\hline
\end{tabular}

Note: The table reports results of the specification in (5). REBP captures the effect of REBP-eligibility on the outcomes listed in columns (1) through (5) on a sample of workers employed at the same establishment in May 1988 and February 1994. We exclude workers born before 1933 and after 1948. Separation denotes an indicator function that is 1 if a worker is not employed by their employer from February 1994 (and May 1988) in February 1996. Separation into Nonemployment denotes an indicator for Separation from the initial employer interacted with an indicator for not being employed in February 1996. Nonemployment (Quarters), Unemployment (Benefits) (Quarters), and Continuous Employment (Quarters) denote the quarters of nonemployment, unemployment benefits, and continuous employment with the initial employer between February 1994 and 1996. Standard errors clustered at the administrative region level are reported in parentheses. Levels of significance: * $10 \%,{ }^{* *} 5 \%$, and ${ }^{* * *} 1 \%$. 
Table 4: Reduced-Form Evidence on Share of Separations within a Coasean Framework Following Perfect Reshuffling vs. Perfect Persistence of Job-Level Surplus: Cohort-Region Cells

\begin{tabular}{lcccc}
\hline \hline & & & & \\
& 1995 & 1996 & 1997 & 1998 \\
\hline$\alpha^{\kappa}$ (Reshuffling) & 1.027 & 1.084 & 1.237 & 1.338 \\
& $(0.034)$ & $(0.030)$ & $(0.040)$ & $(0.047)$ \\
$95 \%$ CI & 0.960 & 1.025 & 1.158 & 1.245 \\
(Lower Limit) & & & & \\
& & & & \\
$\alpha^{1-\kappa}$ (Persistence) & -0.037 & -0.070 & -0.208 & -0.289 \\
& $(0.035)$ & $(0.030)$ & $(0.041)$ & $(0.048)$ \\
& & & & \\
$R^{2}$ & 0.929 & 0.960 & 0.971 & 0.978 \\
$N$ & 181 & 181 & 181 & 181 \\
\hline \hline
\end{tabular}

Note: The table reports estimates of the coefficients in Equation 20. We regress the post-REBP separation rate from February 1994 to February of each year among REBP stayers in the REBP region, by month of birth, on both the separation rate among stayers in the non-REBP region and the predicted separation rate based on a perfect persistence Coasean model. We weight the observations of the month of birth by the number of employed workers born in that month and report standard errors clustered at the administrative region level. 
Table 5: Structural Estimation of Share $\kappa$ of Separations within a Coasean Framework with Perfect Reshuffling of Job-Level Surplus: Industry-Occupation Cells

\begin{tabular}{|c|c|c|c|c|c|c|c|c|}
\hline & \multicolumn{4}{|c|}{ 2-Digit Industry $\times$ Occupation Cells } & \multicolumn{4}{|c|}{ 4-Digit Industry $\times$ Occupation Cells } \\
\hline & 1995 & 1996 & 1997 & 1998 & 1995 & 1996 & 1997 & 1998 \\
\hline \multirow[t]{2}{*}{$\kappa$ (Reshuffling) } & 1.046 & 1.077 & 1.053 & 1.158 & 1.027 & 1.077 & 1.119 & 1.169 \\
\hline & $(0.054)$ & $(0.067)$ & $(0.080)$ & $(0.099)$ & $(0.070)$ & $(0.066)$ & $(0.076)$ & $(0.074)$ \\
\hline $95 \% \mathrm{CI}$ & 0.939 & 0.943 & 0.894 & 0.962 & 0.890 & 0.947 & 0.968 & 1.023 \\
\hline \multicolumn{9}{|l|}{ (Lower Limit) } \\
\hline$R^{2}$ & 0.426 & 0.531 & 0.588 & 0.528 & 0.174 & 0.278 & 0.273 & 0.275 \\
\hline$N$ & 95 & 95 & 95 & 95 & 262 & 262 & 262 & 262 \\
\hline
\end{tabular}

Note: The table reports estimates of $\kappa$ based on estimation of Equation 20] with non-linear least squares allowing for an intercept shift. We collapse the data at the industry by occupation (blue/white collar) level and weight each observation by the number of workers in the cell, dropping cells with fewer than ten workers who survived REBP and those cells with a smaller separation rate in the REBP compared to the non-REBP region during the program (27.8 and $38.6 \%$ of cells for 2-digit and 4-digit industry cuts, respectively). The outcome variable is the separation rate from February 1994 to February of each year among REBP survivors in the REBP region. The model includes the corresponding separation rate among control workers (cohorts born between 1933 and 1943 but ineligible due to location in the control regions) as the main regressor along with its transformations according to Equation 20]. 


\section{Figures}

\section{Figure 1: The Regional Extended Benefit Program (REBP)}

(a) Timeline of Potential Benefit Duration During REBP

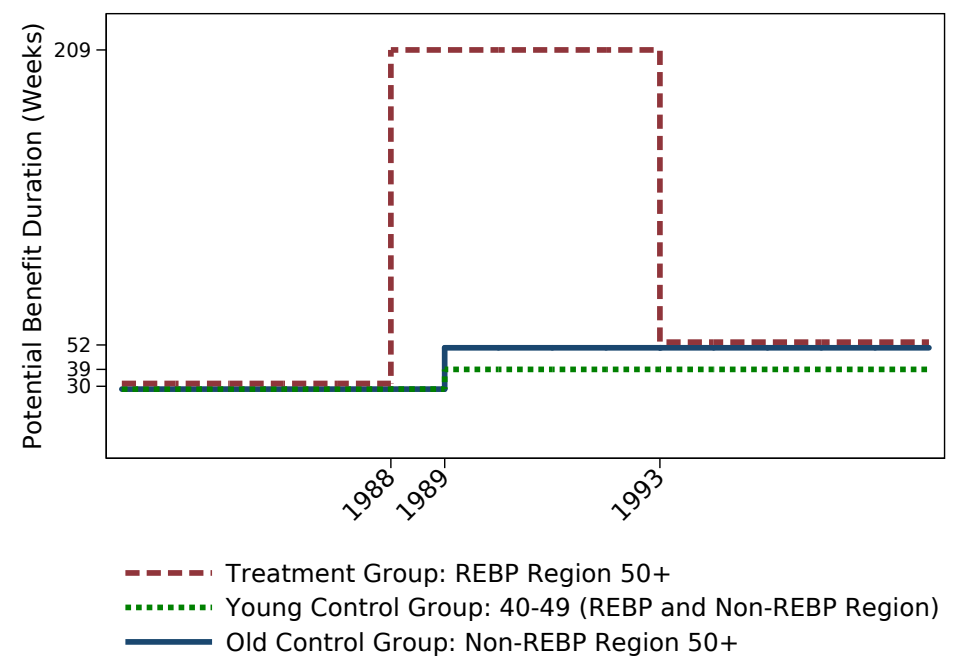

(b) Map of REBP Treatment and Control Regions

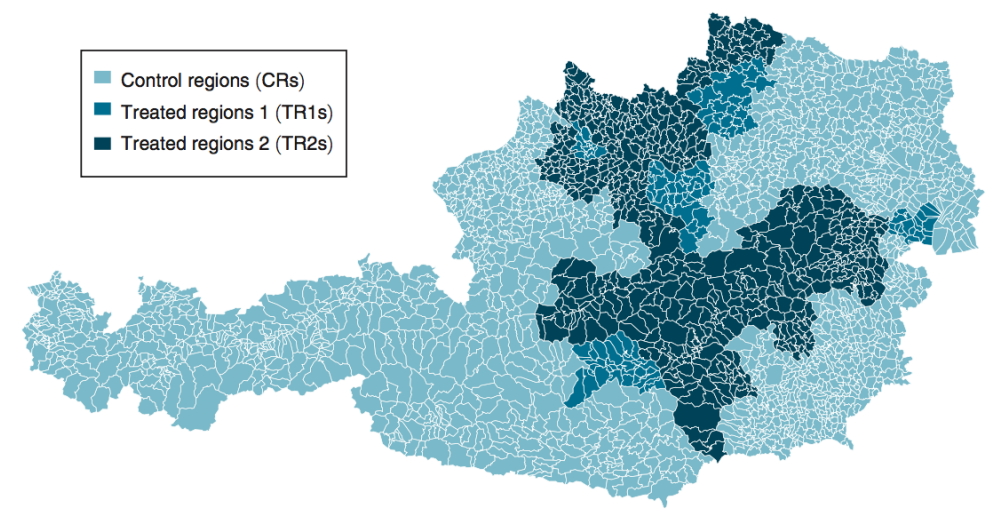

Note: Panel (a) shows the timeline of reform changes in potential benefit duration for eligible workers in REBP and control regions. It shows the maximum length of benefits for individuals aged 50 or older in the highest experience category (at least 9 years during the past 15 years), which increased from 30 to 209 weeks starting July 1988. Individuals who have worked less have a lower maximum benefit duration: If they have worked at least 6 years during the past 10 years, they experience an increase from 30 to 39 weeks in August 1989; if they have worked at least 3 years during the past 5 years, their maximum length of unemployment stays constant at 30 weeks over the whole time period. It also shows the maximum length of unemployment insurance for individuals aged 40-49 who fall into the highest experience category: individuals in this category have worked at least 6 years during the past 10 years. In August 1989, maximum benefit duration increased from 30 to 39 weeks. (The maximum length of unemployment stays constant at 30 weeks for individuals who have worked at least 3 years during the past 5 years.) Panel (b) depicts a map of Austrian municipalities showing the REBP regions. REBP was introduced in TR1 and TR2 in 1988. TR2, REBP was in place until the end of 1991. In TR2, REBP was in place until July 31, 1993. Source for map: Inderbitzin et al. (2016), Figure 1. 


\section{Figure 2: Benefit Extensions and Separations}

(a) Separations (1988 to 1993)

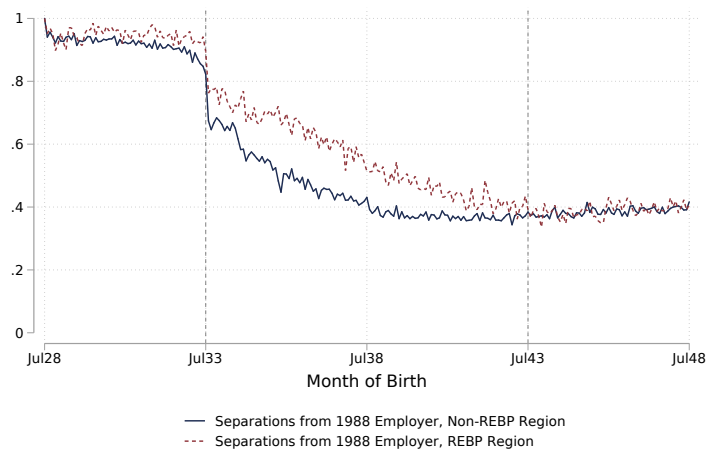

(c) Separations (Ages 50 to 55)

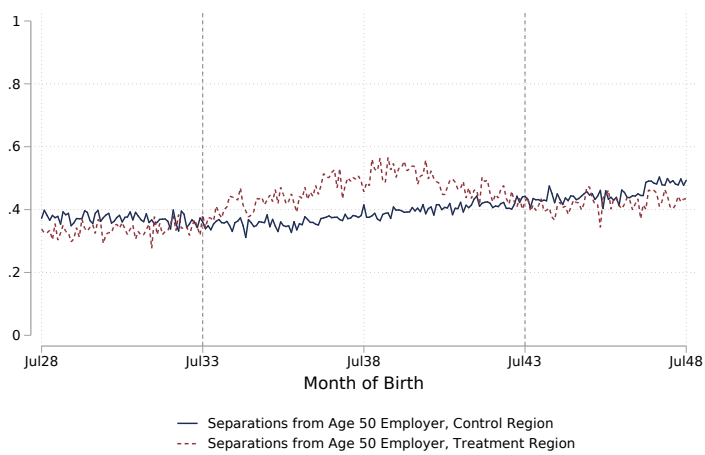

(b) Difference (REBP - Control Region)

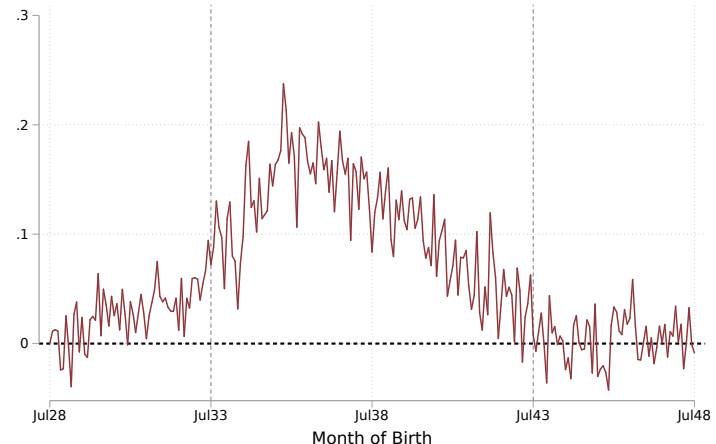

(d) Difference (REBP - Control Region)

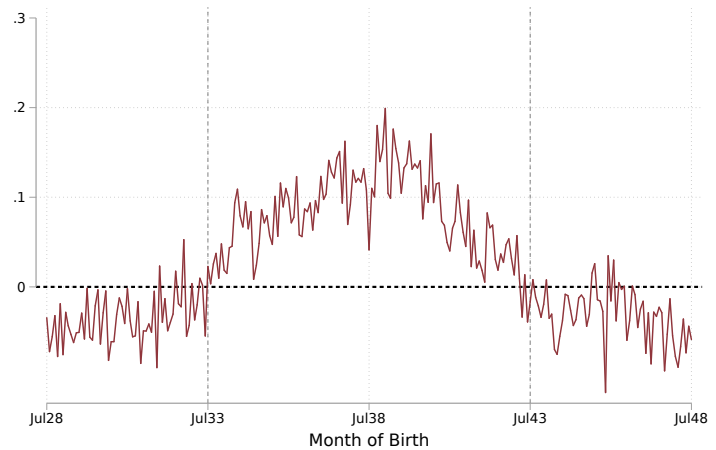

Note: Panel (a) shows the share of workers who separated from their 1988q2-employer (right before the reform) by $1993 q 3$ (when reform had just ended). Panel (c) shows the share of workers who have separated from their employer in the quarter before turning 50 by the quarter before turning 55, i.e. the age range where REBP extended benefits for eligible workers. Both plot rates by month of birth and within the REBP (red, short dashes) and non-REBP (blue, solid) regions. Panels (b) and (d) show the difference between the REBP and the control region by cohort. Cohorts born after 1943 were not covered by the policy as they turned 50 after the program was abolished 1993. 


\section{Figure 3: Benefit Extensions and Employment Outcomes}

(a) Quarters Nonemployed (1988 to 1993)

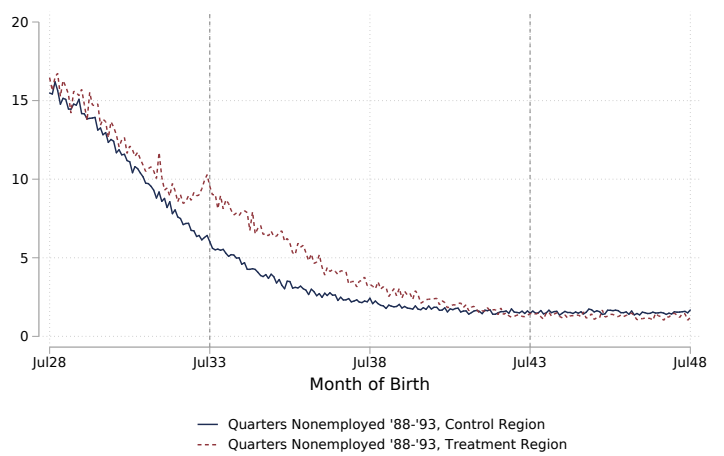

(b) Quarters in Unemp. (Benefits) (1988 to 1993)

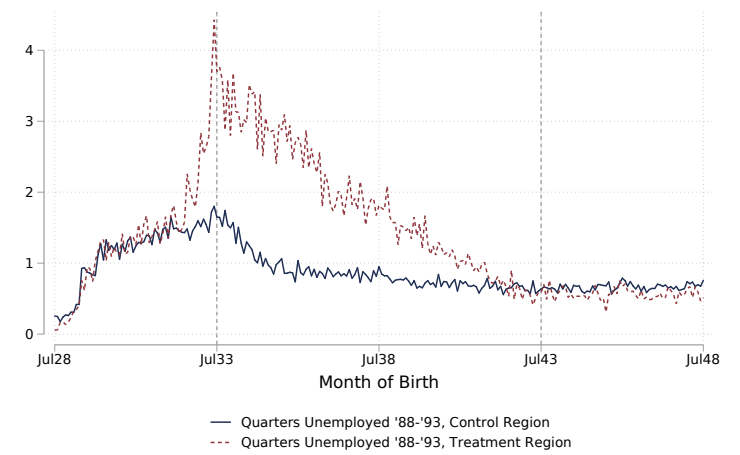

(a) Difference (REBP - Control)

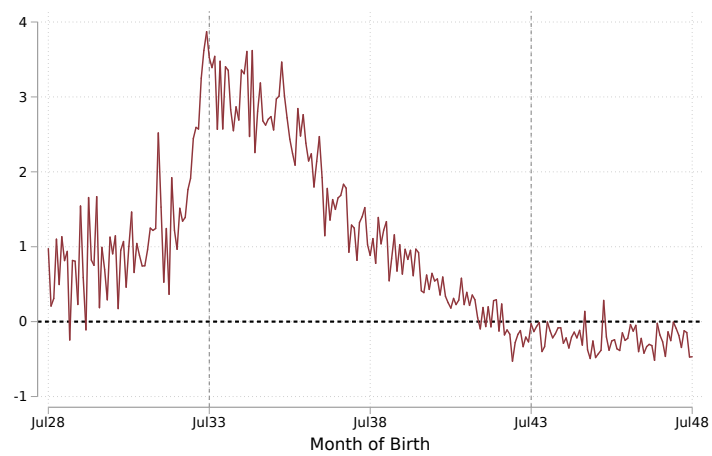

(b) Difference (REBP - Control)

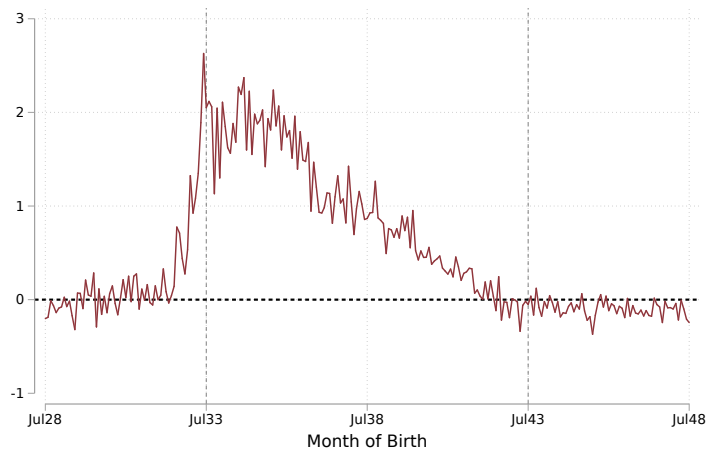

Note: Panels (a) and (c) show the average number of quarters that the workers are nonemployed and on unemployment benefits, respectively, until the quarter after the end of the REBP (1993q3), among those employed in the quarter before the start of the REBP (1988q2). Both plot rates by month of birth and within the REBP (red, short dashes) and non-REBP (blue, solid) regions. Panels (b) and (d) show the difference between the REBP and the control region by cohort. Cohorts born after 1943 were not covered by the policy as they turned 50 after the program was abolished in 1993. 


\section{Figure 4: Complier Characteristics and Treatment Effect Heterogeneity}

(a) Visualizing the Approach

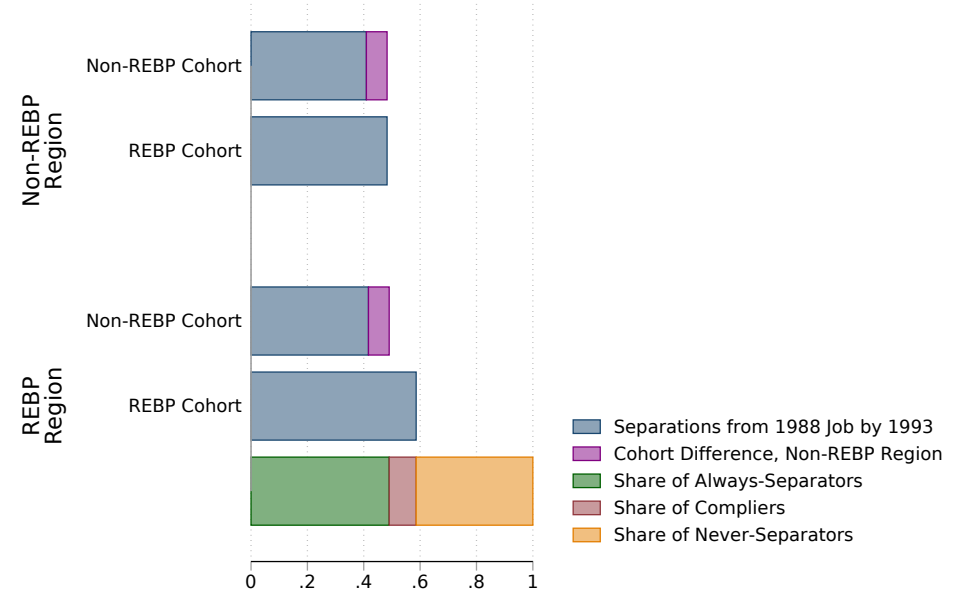

(b) Difference in Characteristics

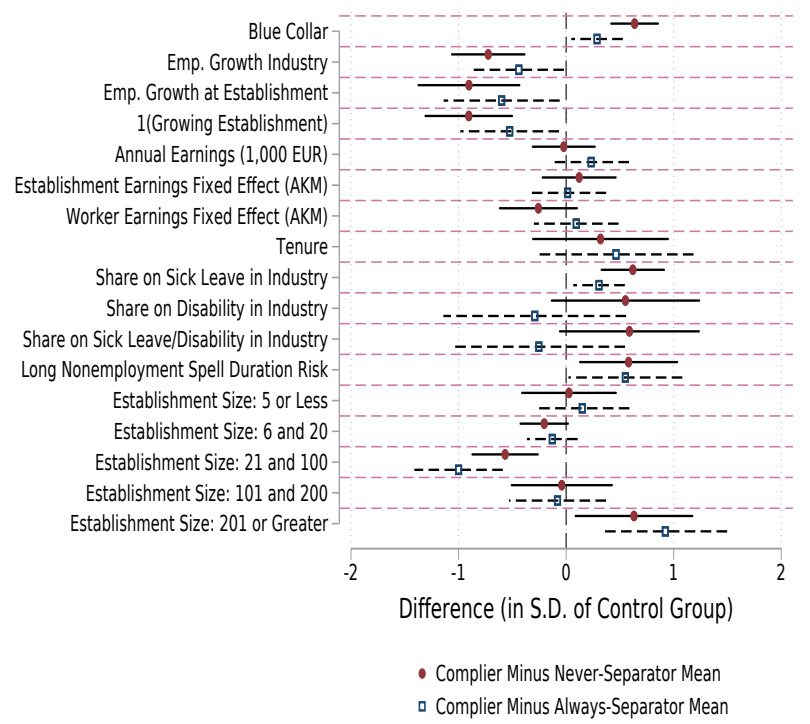

(c) Treatment Effect Heterogeneity

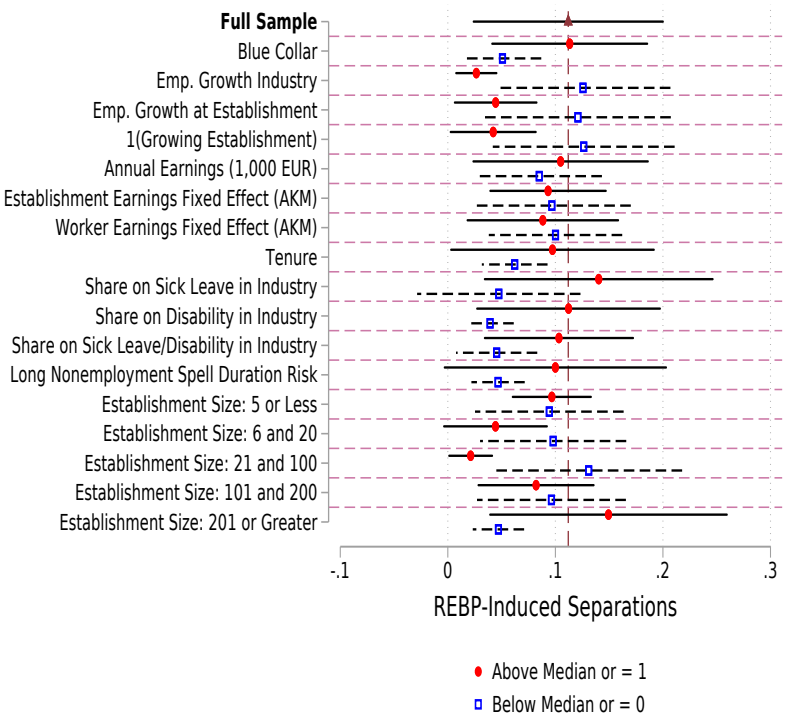

Note: Panel (a) outlines how we create the groups of Always-Separators, Compliers, and Never-Separators using the procedure outlined in Section C It first shows the separation rate among employed workers in 1988q2 by 1993q3, when REBP was abolished, by region and cohort eligibility. It then shows how the share of Always-Separators is made up of the separations among the ineligible age cohorts in the REBP region adjusted for the difference between age cohorts using values from the control region. Any separations in excess of this value are the REBP compliers. Then the share of never-separators are the stayers in the REBP region among the eligible cohort. Panel (b) shows the difference between the averages for compliers and always-separators (C-A) as well as for compliers and never-separators $(\mathrm{C}-\mathrm{N})$ that are reported in Appendix Table A.2 Confidence intervals are based on standard errors from 1,000 bootstrap replications blocked at the administrative region level. See Sections $\mathrm{C}$ and $\mathrm{D}$ for more details on how the variables are constructed. See Section $\mathrm{C}$ for the methodology underlying the decomposition into the groups of compliers, never-separators, and always-separators. Panel (c) shows the heterogeneity in the treatment effect of REBP across different characteristics. For binary characteristics (e.g., Blue Collar), we report the treatment effect for each group ( 1 in red circles, 0 in blue squares). For continuous variables (e.g., earnings), we report the treatment effect on the sample above and below the median (red circles and blue squares respectively). The estimated treatment effect on the full sample is reported at the top with a maroon triangle and the vertical dashed line. 
Figure 5: Intuitions for Empirical Design: Distribution of Job Surplus, Pre- and Post-REBP

(a) Distribution of Job Surplus at the Onset of REBP, both Treated and Control Regions

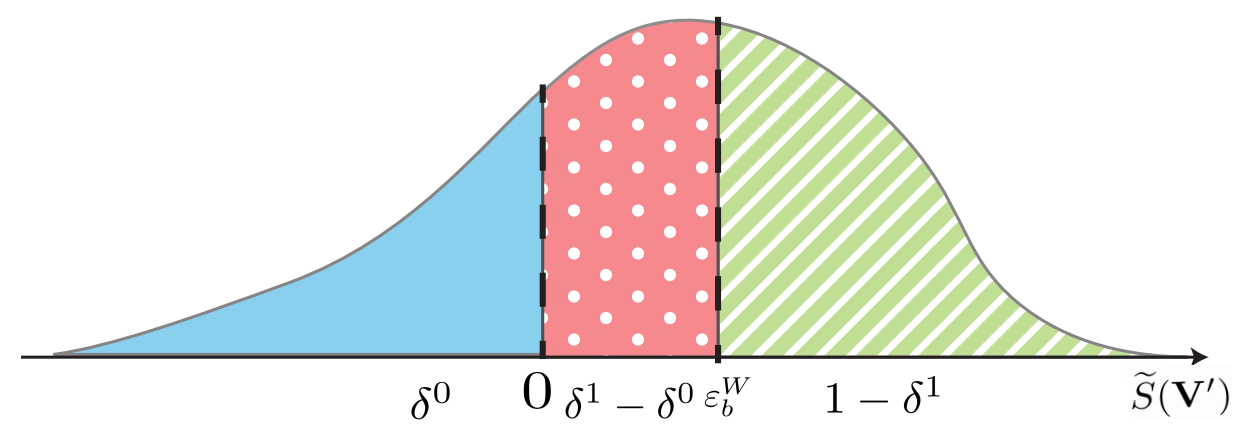

(b) Distribution of Job Surplus at the End of REBP, Treated regions

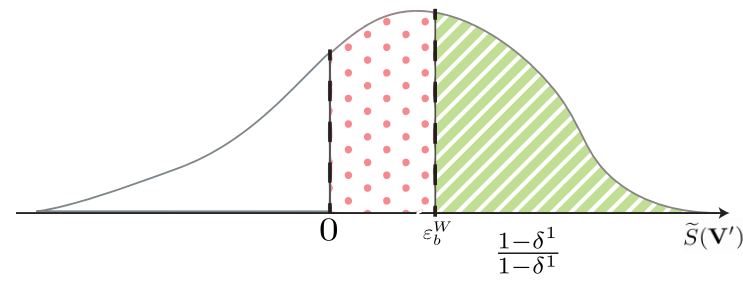

(c) Distribution of Job Surplus at the End of REBP, Control regions

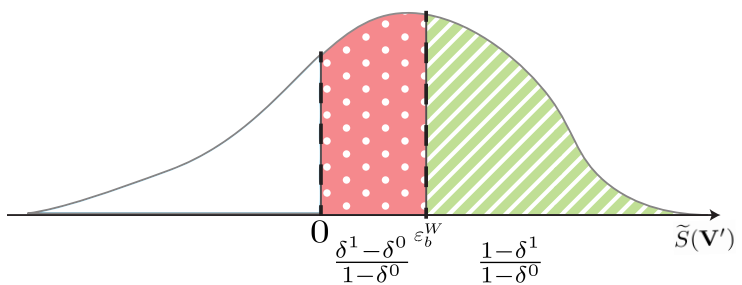

Note: Panel (a) shows a hypothetical distribution of gross-of-REBP joint job surplus realizations during REBP (in terms of the model, after Markov process $k\left(\mathbf{V}^{\prime} \mid \mathbf{V}\right)$ has taken place). The vertical dashed lines represent the zero surplus and the REBP surplus shifter. The light blue solid region to the left of the zero line, making up share $\delta^{0}$ of the total mass, represents the "always separators," meaning those values of gross surplus which will trigger a separation regardless of REBP. The red polka-dotted region between the two vertical lines, making up share $\delta^{1}-\delta^{0}$ of the total, represents the marginal jobs, which separate if subject to REBP but remain active otherwise. The green dashed region to the right of the REBP line, a share $1-\delta^{1}$ of the total, represents the inframarginal active jobs, whose surplus is sufficiently high for feasibility even if subject to REBP. Panels (b) and (c) plot the distributions right after the REBP shock hits (in terms of the model, after the REBP shock but before Markov process $K\left(\mathbf{V}^{\prime \prime} \mid \mathbf{V}^{\prime}\right)$ ), respectively in the treated regions and in the control regions. In the treated regions (Panel (b)), 100\% $\left(=\frac{1-\delta^{1}}{1-\delta^{1}}\right)$ of the surviving jobs have gross surplus greater than the REBP surplus shifter; in the control regions (Panel (c)) fraction $\frac{\delta^{1}-\delta^{0}}{1-\delta^{0}}$ of the surviving jobs have gross surplus between 0 and the REBP surplus shifter (marginal jobs) and a fraction $\frac{1-\delta^{1}}{1-\delta^{0}}$ have gross surplus greater than the REBP surplus shifter (inframarginal jobs). 


\section{Figure 6: Separation Rate of “REBP Survivors" from 1994 Onward}

(a) Through 1995

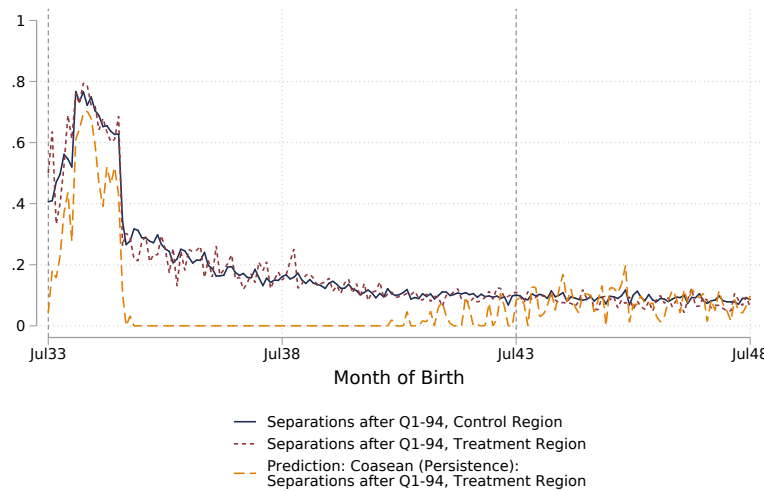

(c) Through 1997

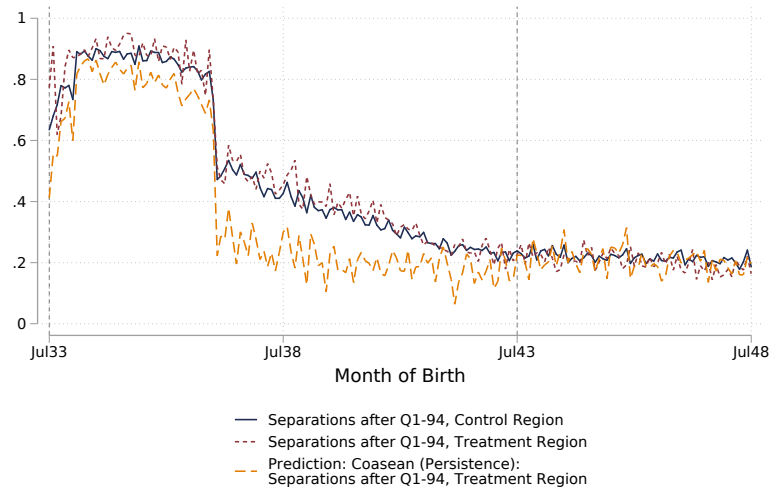

(b) Through 1996

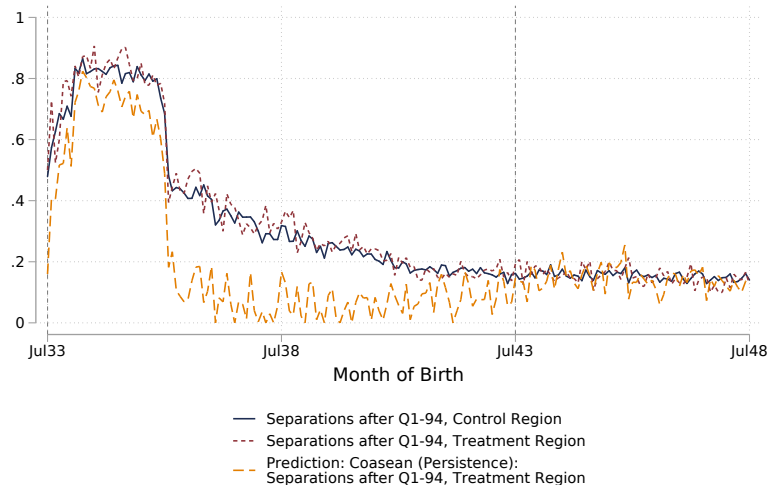

(d) Through 1998

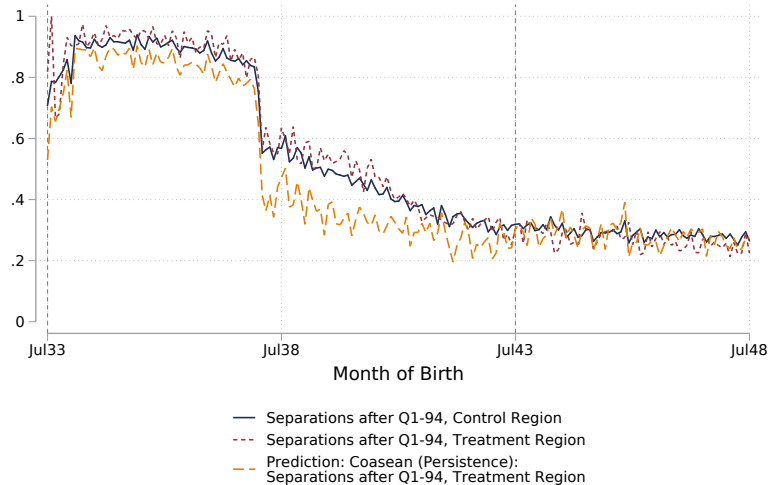

Note: The figure shows, by month of birth, the share of workers observed in the same establishment between 1988q2 and 1994q1 who separate from that employer by Q1 of each subsequent year. The sample is split into REBP (red, short dashes) and non-REBP (blue, solid) regions. The yellow dashed line plots the predicted Coasean separation rate using Equation 18 (perfect persistence case). The retirement age for Austrian men was 60 years old in this period, which explains the spike in separations among older cohorts. 
Figure 7: Difference, REBP vs. Non-REBP Region: Separation Rate of “REBP Survivors" from 1994 Onward

(a) Through 1995

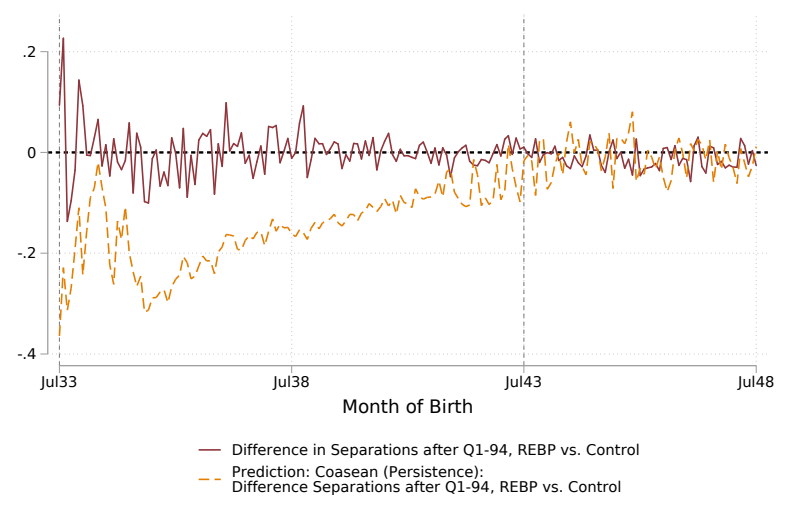

(c) Through 1997

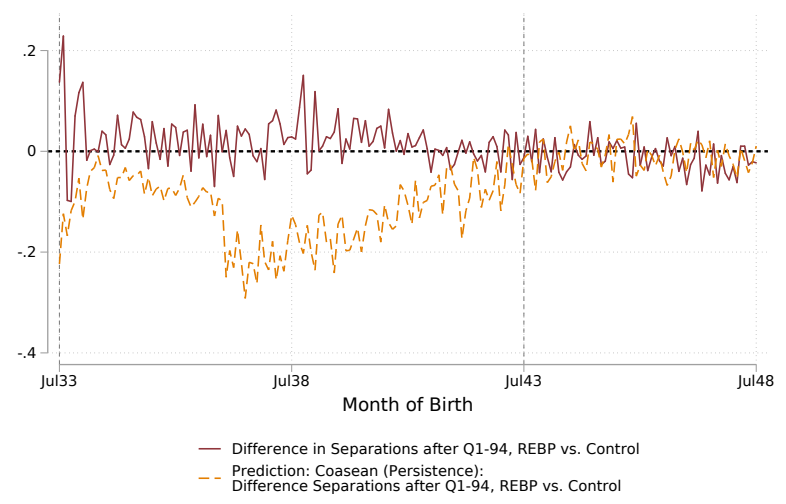

(b) Through 1996

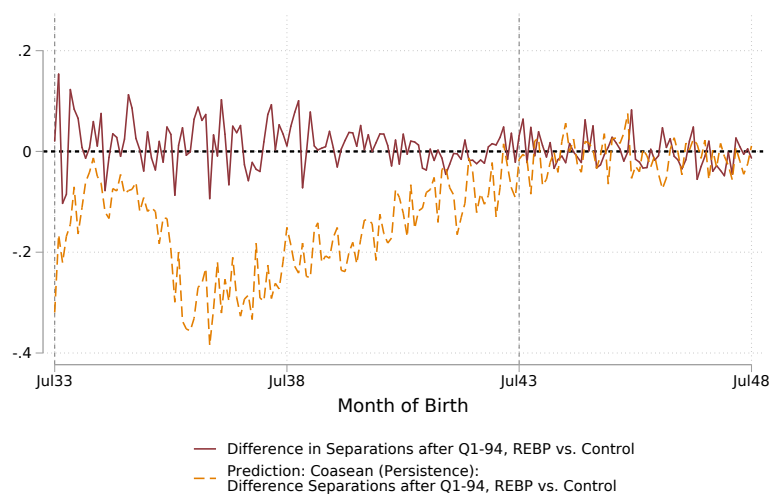

(d) Through 1998

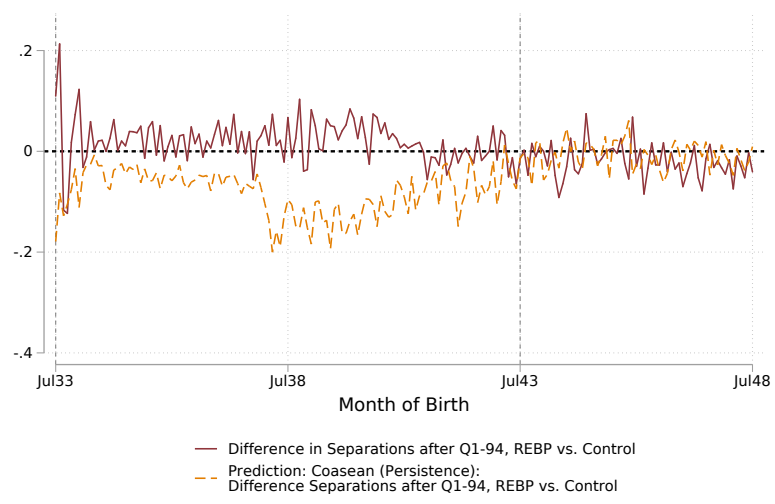

Note: The figure shows, by month of birth, the difference in separation rates from Figure 6 between the REBP and non-REBP regions (red, solid). The yellow dashed line plots the predicted Coasean separation rate using Equation (18) (perfect persistence case). 
Figure 8: Difference by Industry Growth, REBP vs. Non-REBP Region: Separation Rate of "REBP Survivors" from 1994 Onward

(a) Through 1995

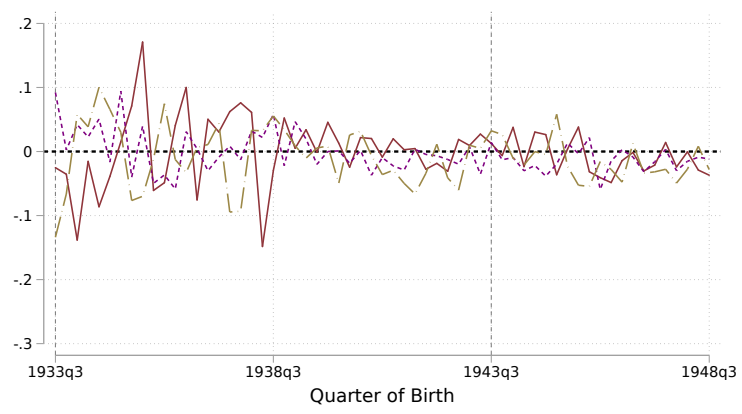

- $\Delta$ Separations after Q1-94, T vs. C, 1st Tercile (Lowest Industry Growth) --- $\Delta$ Separations after Q1-94, T vs. C, 2nd Tercile

- $\Delta$ Separations after Q1-94, T vs. C, 3rd Tercile (Highest Industry Growth)

(c) Through 1997

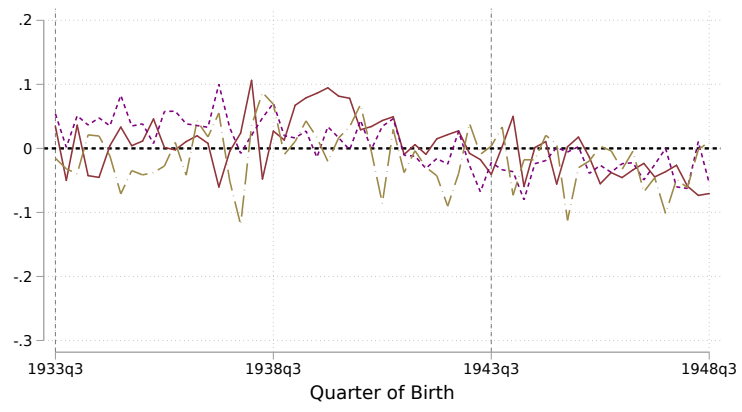

- $\Delta$ Separations after Q1-94, T vs. C, 1st Tercile (Lowest Industry Growth) --- $\Delta$ Separations after Q1-94, T vs. C, 2nd Tercile

- $\Delta$ Separations after Q1-94, T vs. C. 3rd Tercile (Highest Industry Growth) (b) Through 1996

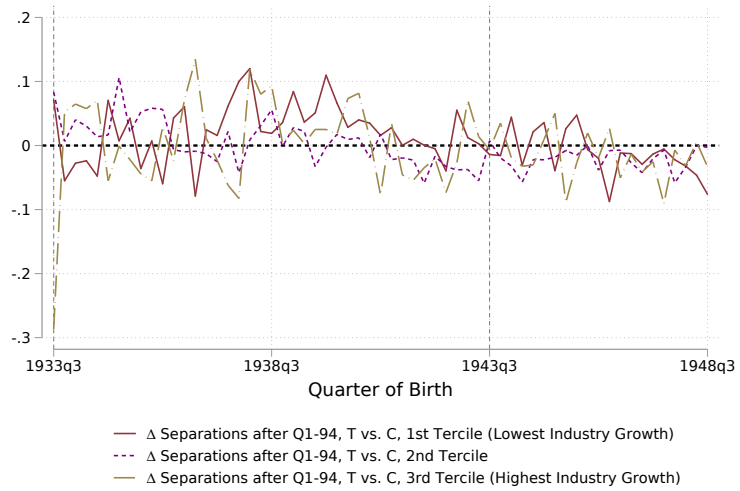

(d) Through 1998

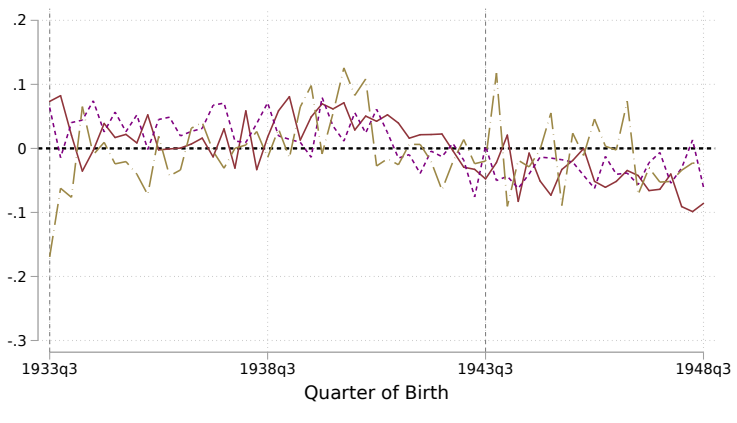

$\Delta$ Separations after Q1-94, T vs. C, 1st Tercile (Lowest Industry Growth) $\Delta$ Separations after Q1-94, T vs. C, 2nd Tercile

- $\Delta$ Separations after Q1-94. T vs. C. 3rd Tercile (Highest Industry Growth)

Note: These figures split the by-cohort regional difference from Figure 7 into terciles of industry growth, with the first tercile denoting the lowest and the third tercile denoting the highest industry growth. Specifically, we calculate employment growth between February 1994 and the given year for each industry, among all workers (not just stayers) born 1938 or later to avoid those who are retiring. We then assign REBP stayers to the industries of their REBP-period establishments. We calculate growth rates for industries at the two-digit NACE level. 
(a) Separations vs. Annual Establishment Growth

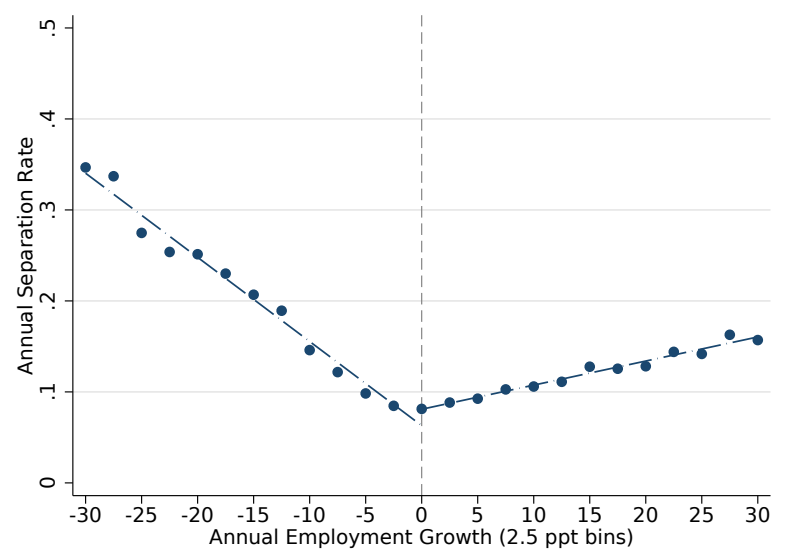

(b) Survivor Separations by Cohort and Region

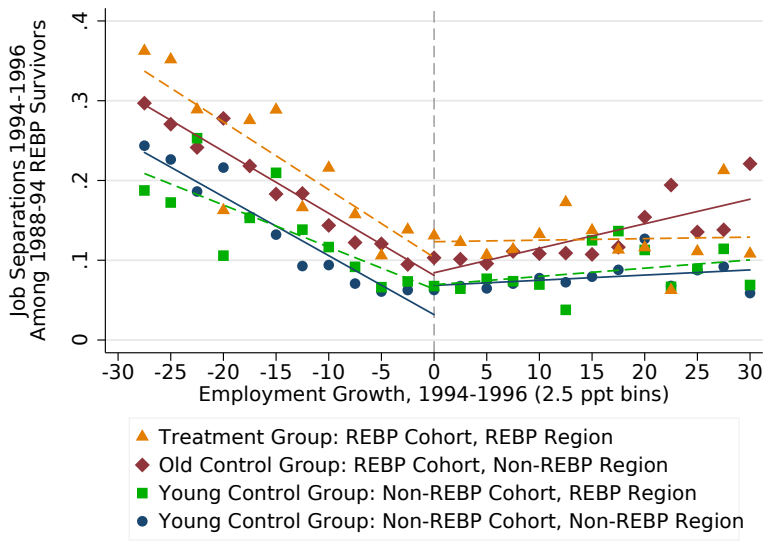

(c) Birth Cohort-Specific Slopes

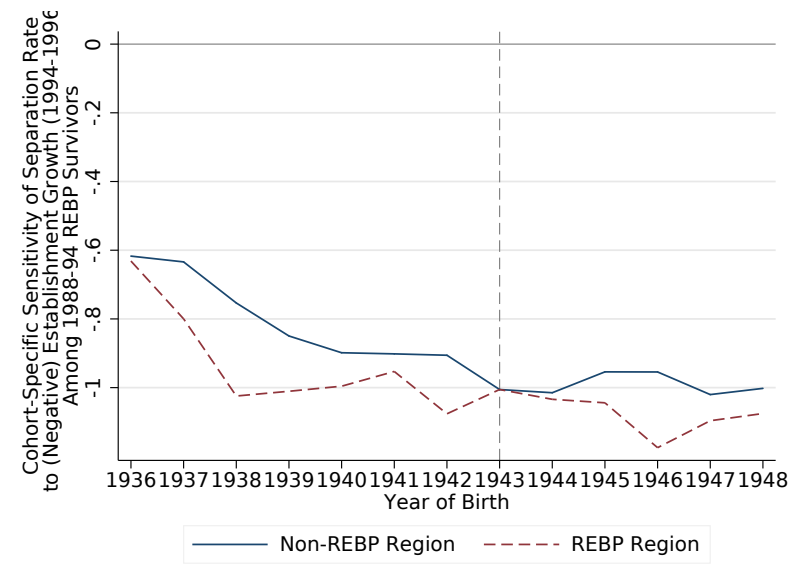

Note: These figures plot the results of an analysis focusing on labor demand shifts within establishments. In an attempt to confirm the "hockey-stick" relationship between separations and employment growth at the establishment level (Davis et al. 2013) in the Austrian setting, Panel (a) plots annual separation rates for male workers employed in a given year by bins of annual establishment employment growth rates (first quarter of every year), pooling years 1994 through 1996 and excluding the steel sector. Panel (b) focuses on separations among the four relevant groups tracked throughout the paper: REBP-eligible and -ineligible birth cohorts and regions, while still plotting against total establishment employment growth. We ignore the cohorts born before 1936 since they have reached retirement age in 1996. Panel (c) plots the slope of the cohort-specific relationship between separations and establishment growth (1994-1996) among shrinking establishments for each year of birth and split between the REBP and non-REBP regions. We adjust throughout for spurious layoffs due to mergers, take-overs, and administrative changes to the ASSD using the procedure outlined in Fink et al. (2010). 


\section{Figure 10: Conceptual Framework (Perfect Persistence): Separations and Shocks}

(a) Case Studies of Jobs

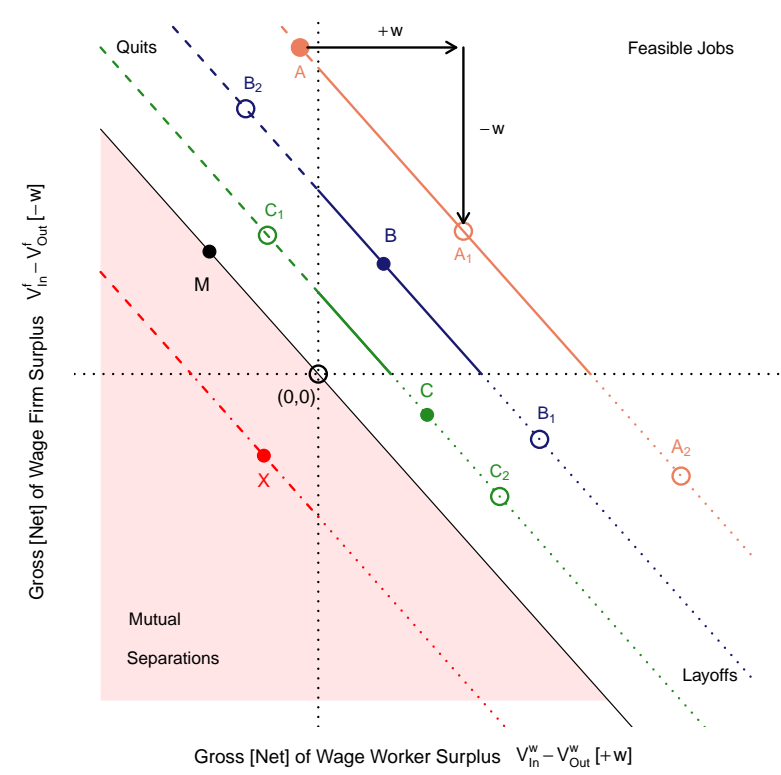

(c) Subsequent Worker Shock

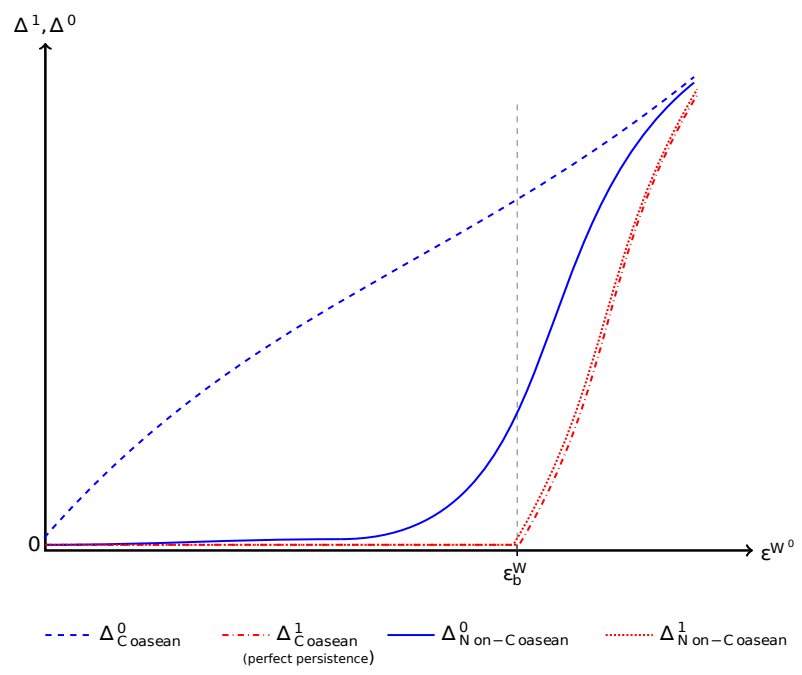

(b) Empirical Strategy: Separation Rates

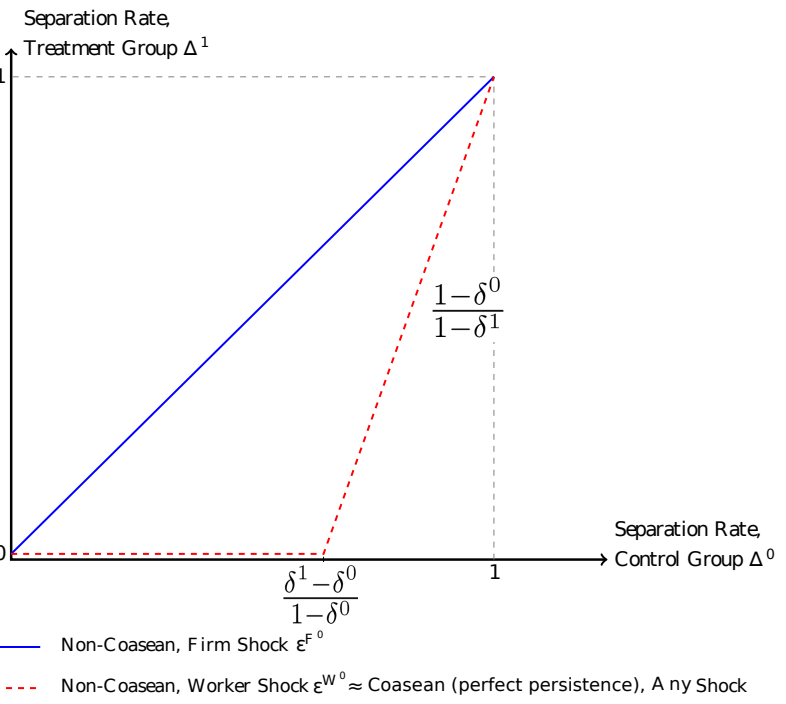

(d) Subsequent Firm Shock

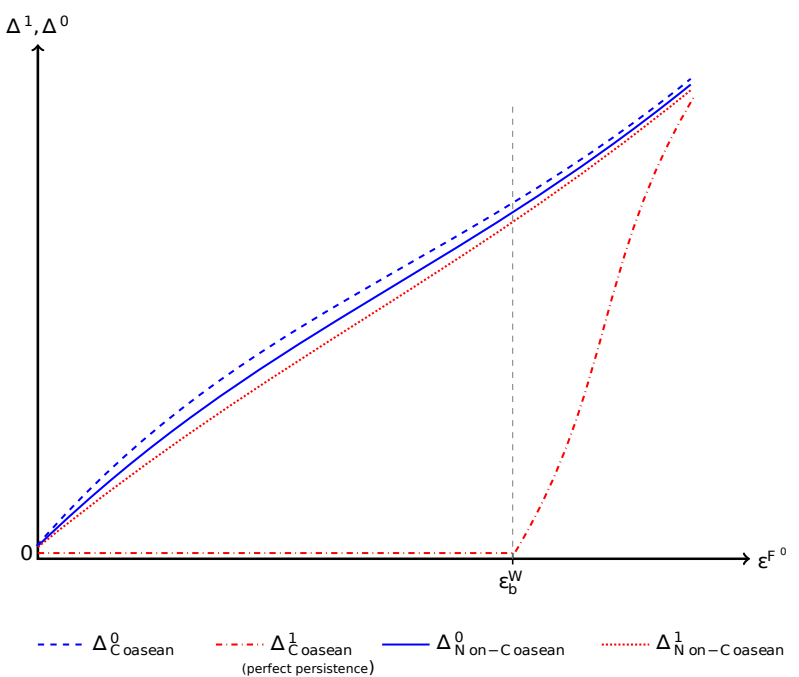

Note: The figures plot the dynamics of job separations in the model, in the Coasean (efficient bargaining) and nonCoasean (fixed-wage) settings. Panel (a) plots job case studies in the two-dimensional space of worker and firm components of joint job surplus, and net of wage surpluses. Panel (b) plots the relationship between treatment group and control group separation rates, after REBP, for the Coasean (assuming perfect persistence of job surplus) and nonCoasean setting. Panels (c) and (d) plot the separations in the former treatment group $\left(\Delta^{1}\right)$ and former control group $\left(\Delta^{0}\right)$ in response to worker (c) and firm (d) side surplus shocks, after REBP, for the Coasean and non-Coasean settings. $\Delta_{\text {Coasean }}^{1}$ is plotted assuming perfect persistence in job surplus between the end of REBP and the subsequent period. Sections 5 and 6 describe the model and the exercises in detail. 
Figure 11: Conceptual Framework: Distribution of Job Surplus and Separations

(a.C) Coasean, UI Benefit Increase

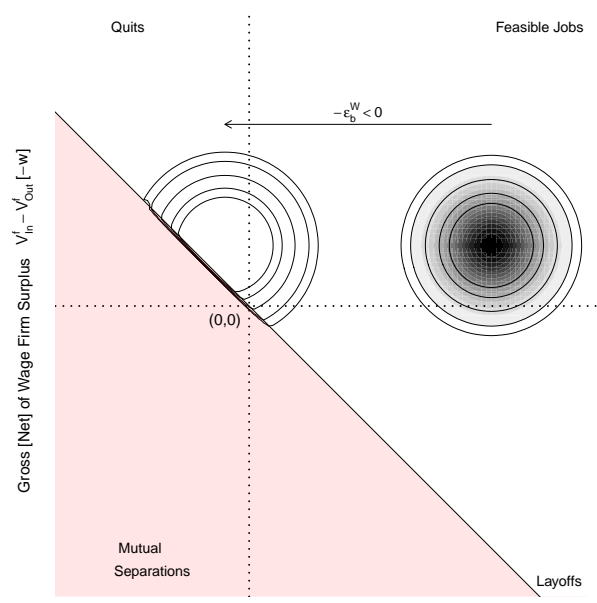

Gross [Net] of Wage Worker Surplus $V_{\text {in }}^{w}-V_{\text {ou }}^{w}[+w]$

(a.NC) Non-Coasean, UI Benefit Increase

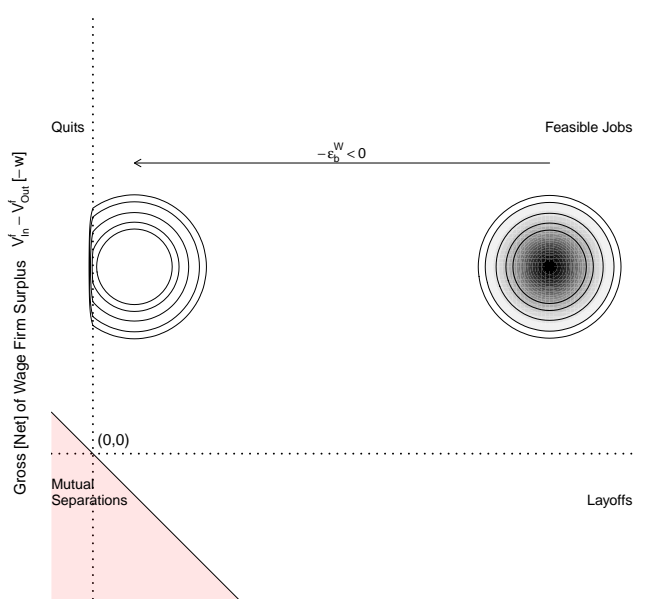

Gross [Net] of Wage Worker Surplus $V_{1 \mathrm{~h}}^{w}-V_{\text {oul }}^{w}[+w]$
(b.C) Coasean, Post-REBP, Treated

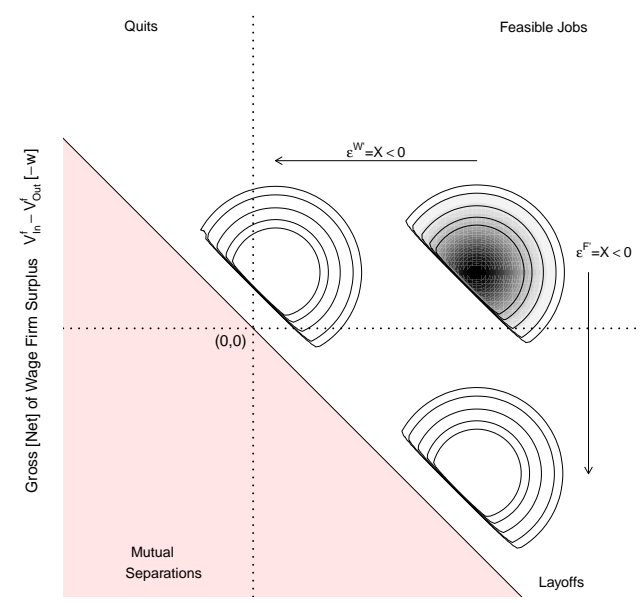

Gross [Net] of Wage Worker Surplus $V_{i n}^{w}-V_{\text {oul }}^{w}[+w]$

(b.NC) Non-Coasean, Post-REBP, Treated

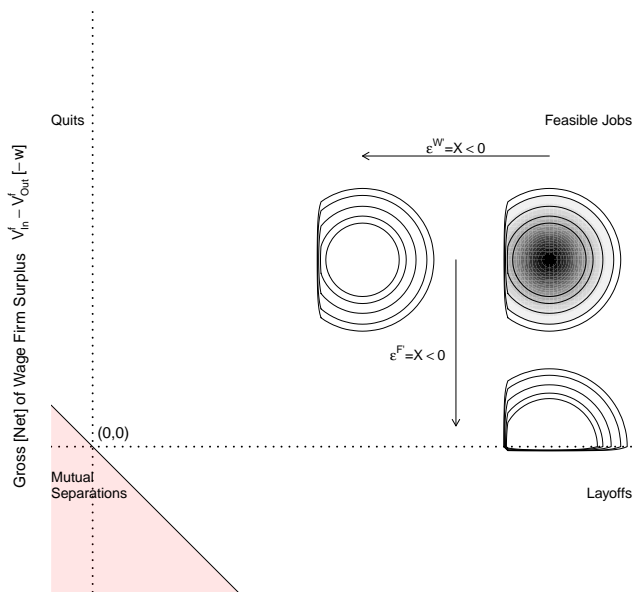

Gross [Net] of Wage Worker Surplus $V_{1-}^{w}-V_{0,1}^{w}[+w]$
(c.C) Coasean, Post-REBP, Control

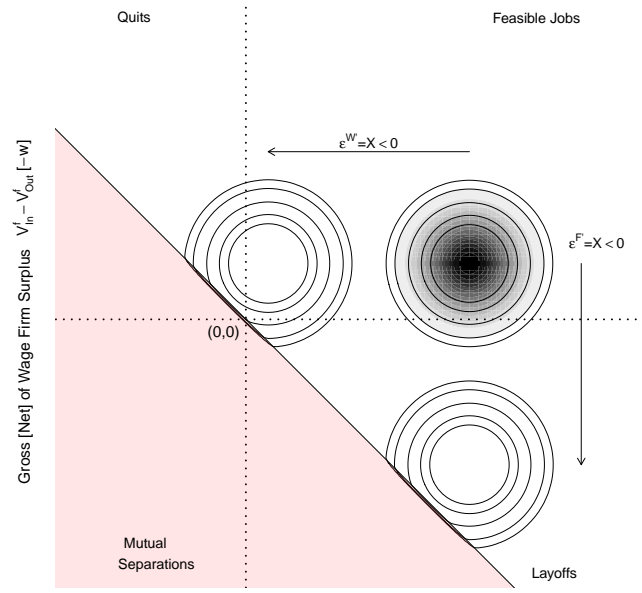

Gross [Net] of Wage Worker Surplus $V_{\text {in }}^{w}-V_{\text {Oun }}^{w}[1+w]$

(c.NC) Non-Coasean, Post-REBP, Control

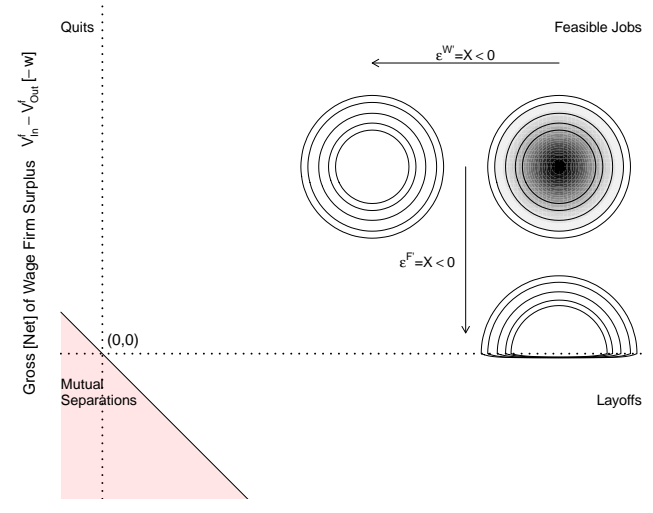

Gross [Net] of Wage Worker Surplus $v_{\text {in }}^{w}-V_{\text {oul }}^{w}[+w]$

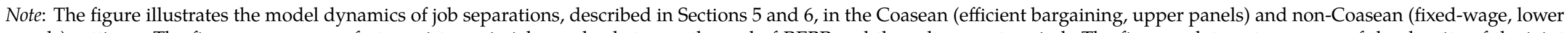

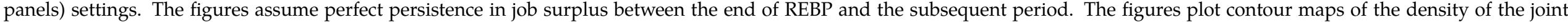

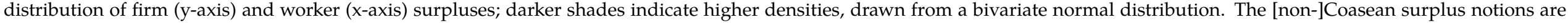

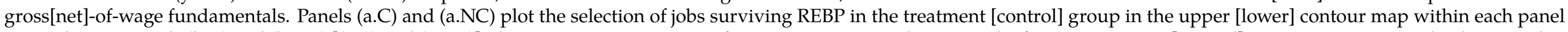

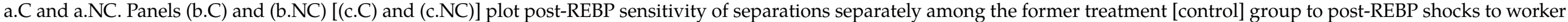
and firm surplus. 
Figure 12: Post-REBP Separation Rates of REBP Survivors and non-REBP Survivors at the IndustryOccupation Level

(a) Separations by 1995

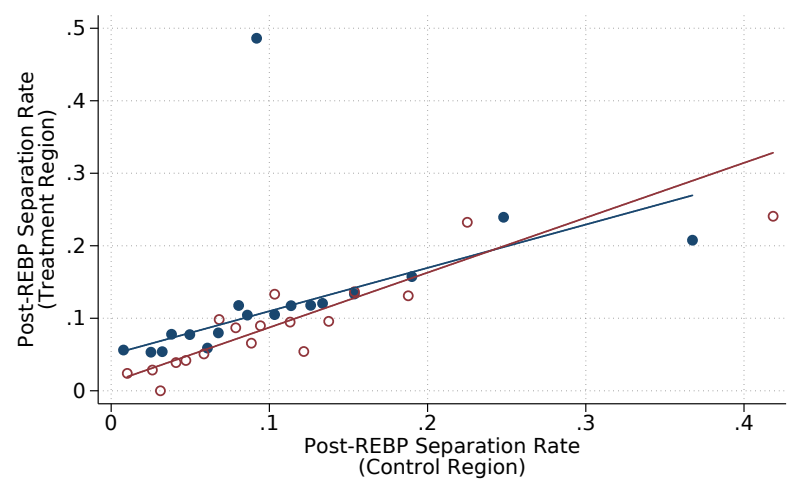

- Formerly Treated Cohorts ('38 to '43)

- Younger Control Cohorts ('43 to '48)

(c) Separations by 1997

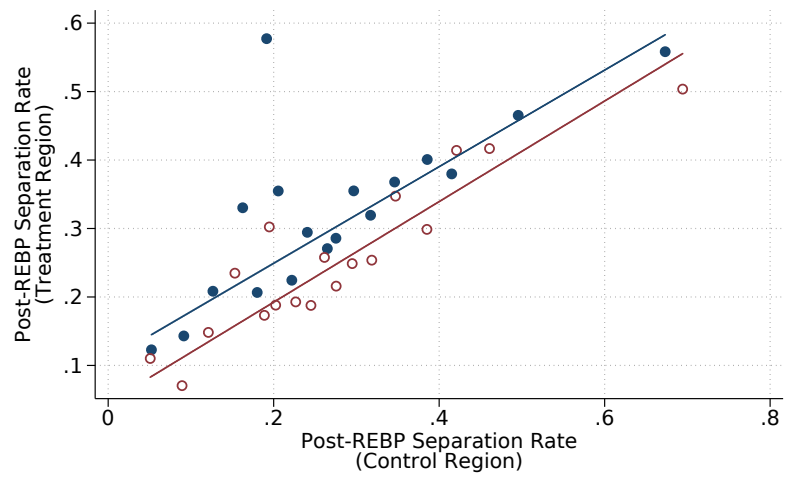

- Formerly Treated Cohorts ('38 to '43)

- Younger Control Cohorts ('43 to '48) (b) Separations by 1996

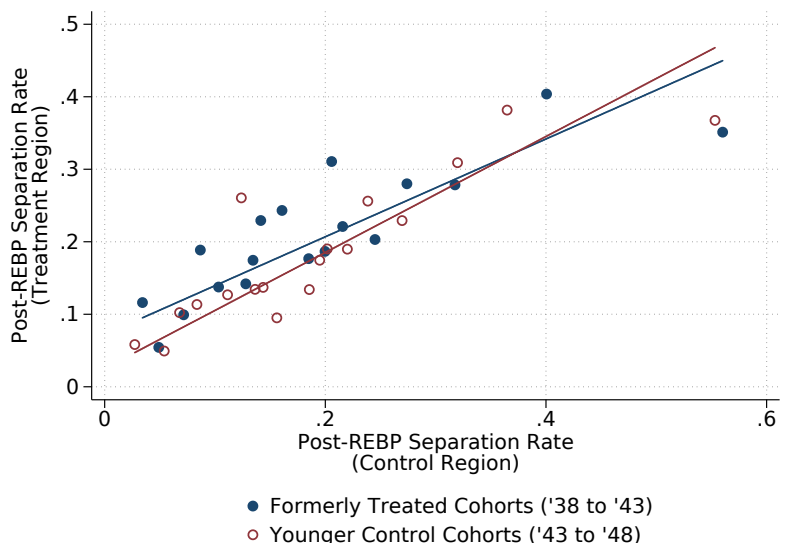

(d) Separations by 1998

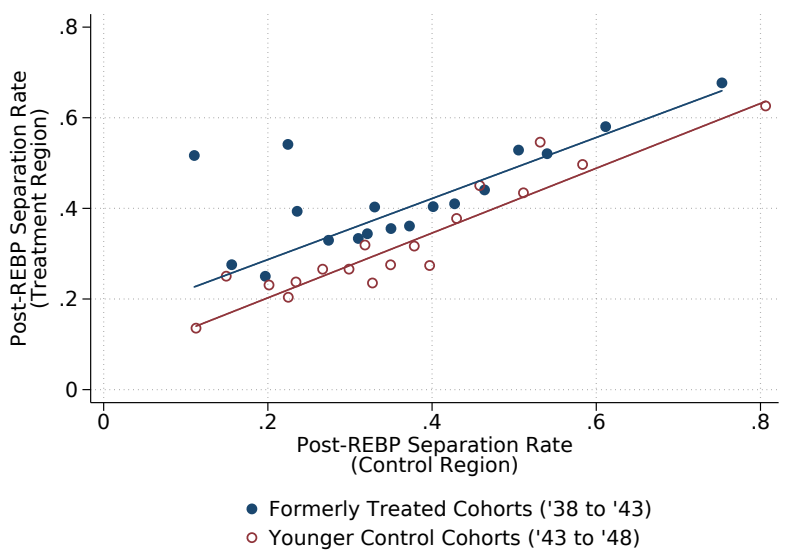

Note: The figures plot binned scatter plots of the post-REBP separation rates at the industry-by-occupation cell level among survivors in the REBP regions against those in the same cell in the non-REBP regions. The blue dots indicate the relationship for cohorts born between 1938 and 1943, i.e. those who were eligible for the REBP before its abolition. As a placebo, we also show the relationship for slightly younger cohorts, born between 1943 and 1948 and thus never eligible for the REBP. Observations are weighted by the number of survivors in the respective control-region cell. Industries are categorized at the four-digit level. 
Figure 13: Separation Effects, Resilience and Control Region Separation Rates By Predicted LongTerm Nonemployment Risk

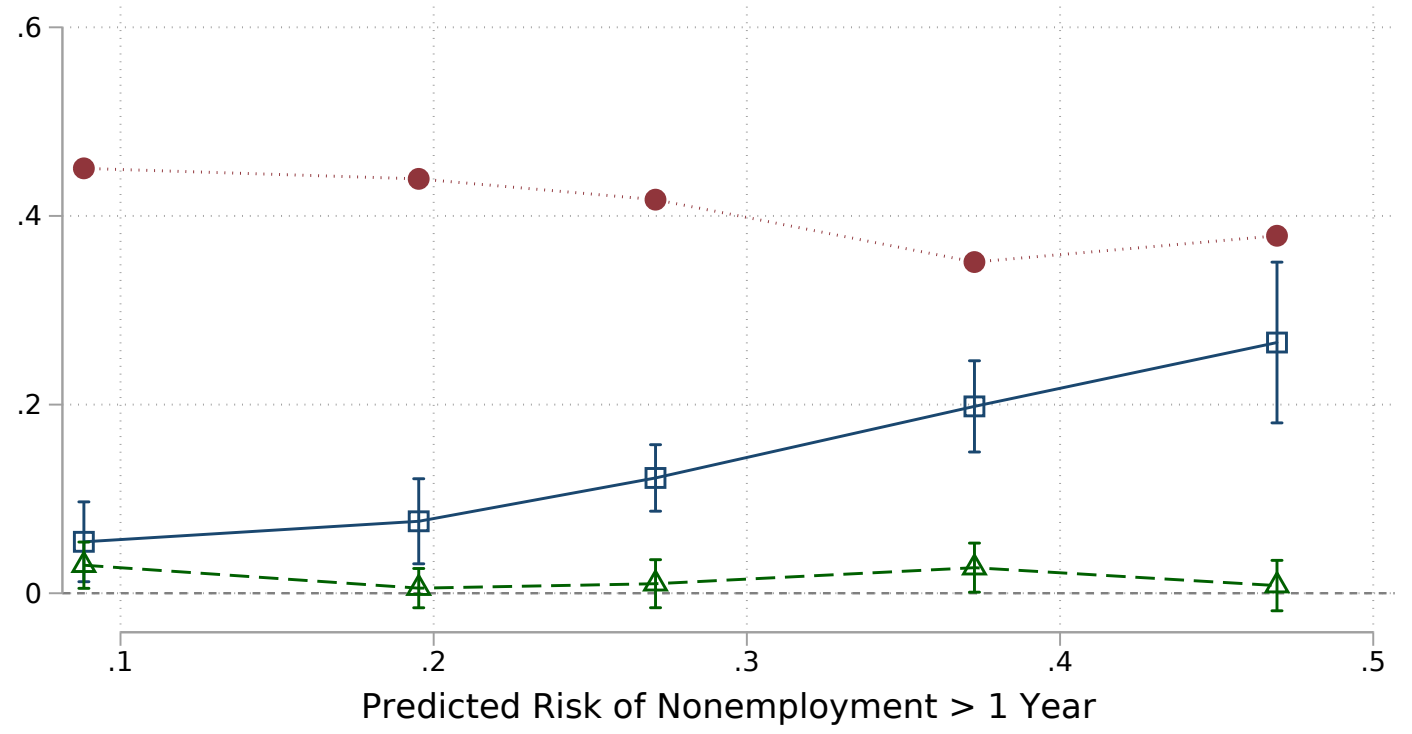

$\square$ REBP Effect on Separations
$\triangle$ Effect on Survivor Separations (Post-Abolition)
- Control Region Separation Rate (During REBP)

Note: The figure plots separation effects, estimated resilience and control region separation rates by quintiles of predicted nonemployment risk. We calculate nonemployment risk by selecting the set of workers employed in 1982 and measure whether they experienced a long nonemployment spell by 1987 (i.e. before the REBP reform). In this sample, we regress an indicator for experiencing a consecutive nonemployment spell of more than one year on (15) industry fixed effects, an indicator for white-collar occupations, cubic polynomials in tenure and experience (in the last 25 years), and the local unemployment rate at the municipal level. We then use the estimated coefficients to predict long-term nonemployment risk among the sample of workers employed in 1988 before the REBP became active and group workers into five quintiles based on this measure. The navy hollow squares plot the effect of the REBP on separations in each of the five cells. The green hollow triangles are the post-REBP separation effects among survivors by 1995 (negative value would indicate resilience). Finally, the maroon solid circles plot the control region separation rate among peer old cohorts in the non-REBP region (during the REBP). 


\title{
Online Appendix of:
}

\section{Marginal Jobs and Job Surplus: A Test of the Efficiency of Separations}

\author{
Simon Jäger, Benjamin Schoefer, and Josef Zweimüller
}

\section{A Quantifying Worker's Value of the REBP UI Extension}

We calculate the cash value of extended benefits following the approach in Card et al. (2007) and complement it with new data on unemployment assistance (UA, "Notstandshilfe" in German). Our estimate for the average cash value of the reform corresponds to about eight to nine months of pay or $71 \%$ of a worker's annual salary.

The REBP changed potential UI benefit duration from 30 or 52 weeks to 209 weeks for older workers (see Figure 1 Panel (a)). ${ }^{59}$ To provide a conservative estimate of the value of the reform, we take 52 weeks as the alternative potential benefit duration. Under this assumption, REBP changed benefits by 157 weeks or 36.1315 months.

When benefits run out, many workers are eligible for lower UA benefits. UA benefits are meanstested and depend on other (spousal) sources of income as well as the number of dependents. They are capped at 0.92 of the worker's UI benefits, according to the formula

$$
\mathrm{UA}=\min \{0.92 \times \mathrm{UI}, \max \{0,0.95 \cdot \mathrm{UI}-\text { Spousal Earnings }+ \text { Dependent Allowances }\} \text {. }
$$

To impute counterfactual UA payments, we draw on data from the AMS, the Austrian employment agency, on unemployment benefit and UA receipt. This allows us to observe actually paid out UI and UA benefits. We draw on data from a period when both UI and UA payments are observed in the AMS data (2001-2009), and zoom in on workers whose UI benefits ran out and who did not take up employment in the subsequent 60 days. We then calculate the average ratio of UA to UI benefits. We assign everyone zero UA benefits if they do not receive UA benefits in the 60 days after UI benefits ran out, even though they may have been eligible for non-zero UA benefits but did not take them up. In our sample, we find that the average UA benefit corresponds to $50.5 \%$ of previous UI benefits.

The average replacement rate between 1988 and 1993 was 40.0\%. We calculate the average replacement rate for workers in eligible cohorts in the REBP region by simply assigning replacement rates to workers based on their earnings and averaging over workers from 1988 to 1993.

As a final input into our calculation, we account for the fact that benefits are not taxed. The average tax rate for personal income in Austria was 11.2\% after a 1989 tax reform (OECD, 1990). In addition, employee-borne payroll taxes of about $18 \%$ were levied on wages ${ }^{60}$ We thus scale up UI and UA benefits relative to gross income by $1 /\left(\left(1-\tau_{\text {average }}\right)\left(1-\tau_{\text {average }}^{\text {Soc. Sec. }}\right)\right)$ to account for non-taxation of benefits.

\footnotetext{
${ }^{59}$ For most of the treatment period, since 1989, the potential benefit duration for older workers was 52 weeks. Until 1989 , the potential benefit duration was 30 weeks.

${ }^{60}$ Specifically, the total payroll taxes contribution rates for workers and firms were, in sum, 34.5\% for blue- and $38.6 \%$ for white-collar workers (OECD, 1990). In our sample, about 35.4\% of workers among 1988 job holders were white-collar workers so that the average social security contribution rate is $0.345 \cdot(1-0.354)+0.386 \cdot 0.354 \simeq 0.36$, leading to a worker contribution rate of $18 \%$.
} 
We can then calculate the cash value of the reform to the average worker according to the formula:

$$
\underbrace{31.1315}_{\text {Additional UI months }} \times \underbrace{0.400}_{\text {UI RR }} \times \underbrace{(1-0.505)}_{\left(\frac{\text { UI RR - UA RR }}{\text { U RR }}\right)} \times \underbrace{(1-0.115)(1-0.18))^{-1}}_{\left(\left(1-\tau_{\text {average }}\right)\left(1-\tau_{\text {average }}^{\text {Soc. }}\right)\right)^{-1}} \times w \simeq 8.494 \cdot w,
$$

where $w$ denotes the average worker's monthly gross wage and RR denotes replacement rates. According to this calculation, the average cash value of the REBP reform to workers was about eight to nine months of salary or $71 \%$ of an annual salary: ${ }^{61}$

${ }^{61}$ Wages in Austria are paid based in 14, rather than 12, installments. The additional two installments are incorporated in the calculation of UI benefits. The monthly wage we mention above corresponds to an average wage corresponding to the annual salary divided by 12. 


\section{B Worker Survey}

To complement our model-driven test that sorted workers by the economic concept of job surplus, we provide evidence on classifications of REBP separations into subjective categories reported by workers themselves.

\section{B.1 Classifying REBP Separations: Survey and Administrative Data}

We now study micro-level survey data and an administrative measure to study the nature of the separations induced by the outside option shift. We find that workers largely perceived these separations to be amicable quits and early retirement.

Survey: Micro Census The Austrian Micro Census ("Mikrozensus") is the largest continuous survey of the Austrian population. The Austrian Micro Census samples representatively based on administrative population registries and follows a rotating panel of households. We additionally use this survey to trace the nature of separations by type (quits, layoffs, other reasons) as it contains information on the reasons for the ends of nonemployed worker's last job, starting in 1995. For jobs that ended within the last eight years, respondents report the reason for the separation as follows:

1. a one-sided or amicable quit,

2. a layoff due to establishment closure,

3. a layoff due to economic reasons,

4. a layoff due to other reasons,

5. early retirement, incl. limited employability and health 62

6. regular retirement, or

7. other reasons (incl. expiration of fixed term contracts, civil service, and a residual, unclassified category).

As a basis for this analysis, we replicate the REBP treatment effect on separations in the micro census sample.

Validation of Micro Census: Replicating the Treatment Effect of REBP on Separations We validate whether the REBP led to excess separations in our micro census sample. Figure A.1 plots an indicator for whether the last employment spell of a nonemployed respondent ended when the respondent was between 50 and 54 years old, the effective age range when extended benefits were available under REBP. The Figure shows averages of the indicator by cohort and region. As in the analysis based on administrative data, the analysis based on the Microcensus survey data clearly documents excess separations in the REBP regions for cohorts affected by the reform - those born between 1933 and 1943. The lines of the REBP and the control regions are parallel and in fact almost lie on top of one another outside of the treatment cohorts.

\footnotetext{
${ }^{62}$ The questionnaire and survey manual clarify that "early retirement" is supposed to encompass these additional categories.
} 
Figure A.1: Microcensus: Effect of REBP on Separations between Age 50 and 54

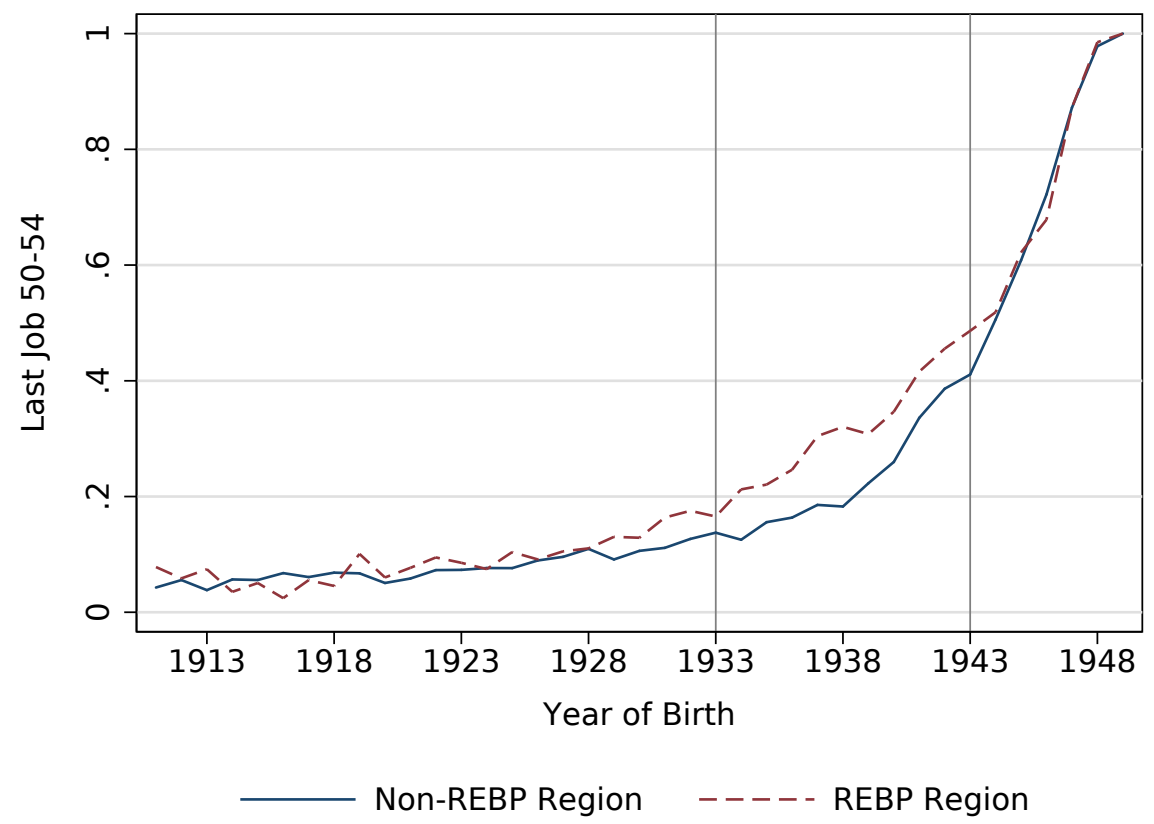

Note: The figure plots data based on the Austrian Microcensus. Across cohorts and regions, it plots an indicator variable for whether a respondent's last employment spell ended in the time when a respondent was between 50 and 54 years old. The two red vertical lines denote the oldest and youngest cohorts, respectively, who were eligible for REBP between 1988 and 1993 and were aged between 50 and 54 at some point in that time range.

Composition Shifts in Separation Types We focus on the sample of respondents whose job ended when they were between 50 and 54 years old. We estimate a difference-in-differences model with cohort and region effects from Equation (1), with indicator variables for last job ending between the ages of 50 and 54 in a respective separation type.

Figure A.2(a) plots the difference-in-difference treatment effects of REBP on these seven types of separation indicators and documents that the largest categories of separation types are classified as early retirement and retirement for other reasons as well as quits, including amicable quits. Specifically, we find a statistically significant, positive effect of REBP on quits among older workers of about $0.7 \mathrm{ppt}$ and effects of about $1.5 \mathrm{ppt}$ for both early retirement and retirement for other reasons. Figure A.2 (a) also provides some evidence for a small and positive effect on layoffs due to economic reasons with an effect size of about $0.5 \mathrm{ppt}$, about a tenth of the overall increase in separations. The effects on layoffs due to closure or for other reasons are much smaller, and statistically not significant. In a next step, we dissect the reasons for the additional quits into (i) personal or family circumstances, (ii) sickness or disability, or (iii) other reasons (Figure A.2(b)). Strikingly, almost half were due to sickness or disability. 


\section{Figure A.2: Effect of REBP on Quits and Mutual Separations between Age 50 and 54}

(a) Microcensus: Effect on Separation Types

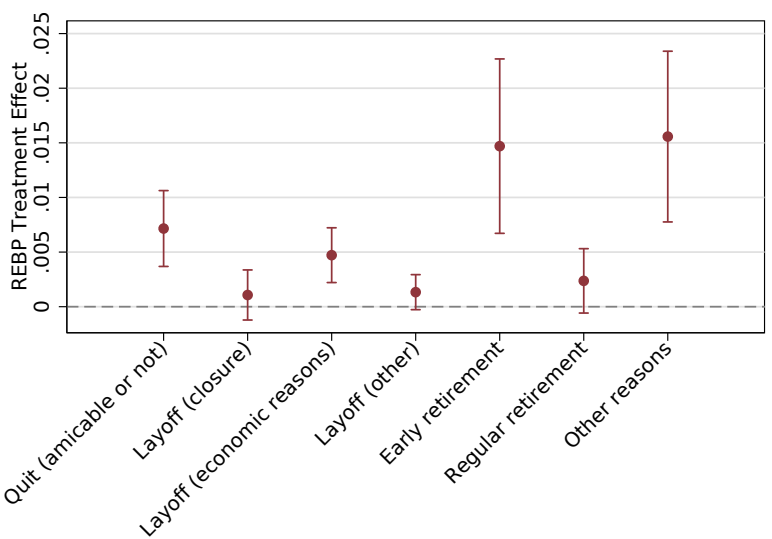

(c) ASSD: Effect on Quits

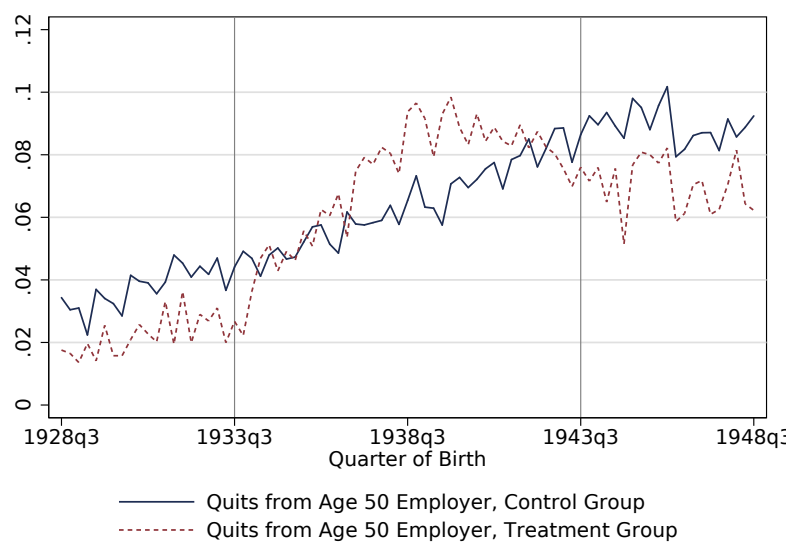

(b) Microcensus: Effect on Types of Quits

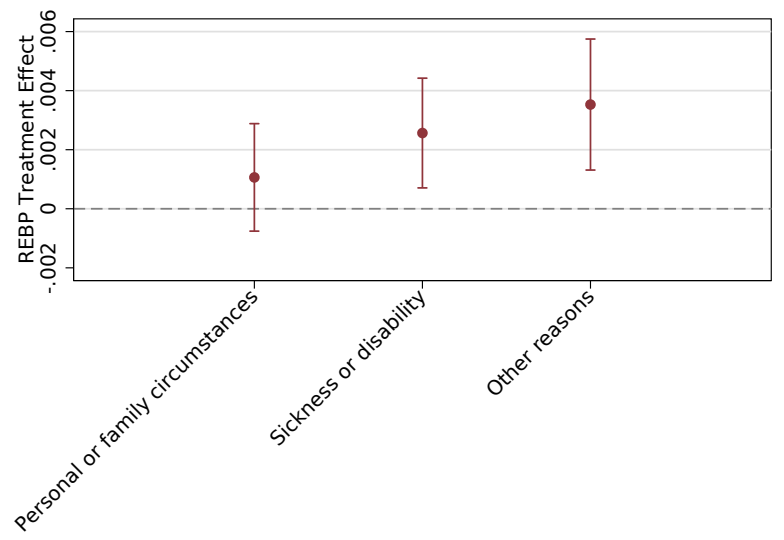

(d) ASSD: Difference (REBP - Control)

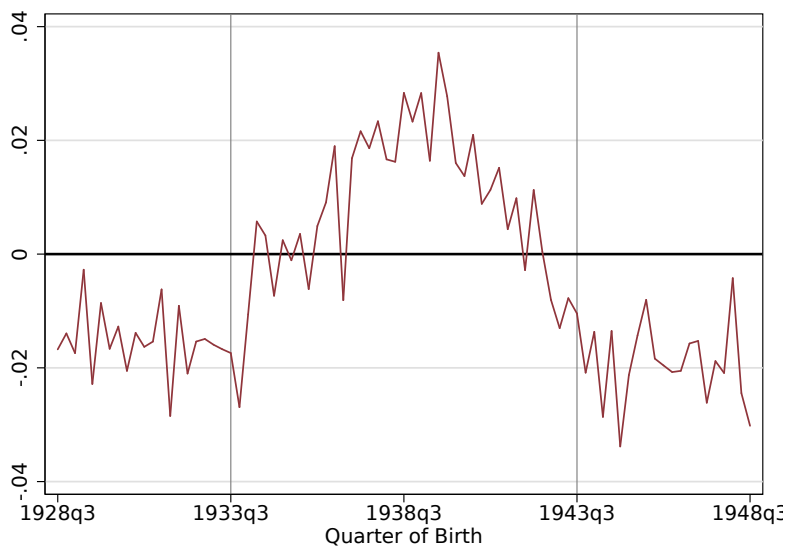

Note: The figures plot data on quits from two sources. Panels (a) and (b) plots outcomes based on the Austrian Microcensus and show treatment effects for REBP in a difference-in-difference specification controlling for region and cohort fixed effects on types of separation (a) and, more specifically, types of quits (b). The seven outcome variables that we consider in (a) are indicators that equal one if a respondent's last employment spell ended between the ages of 50 and 54 and was either a one-sided or amicable quit, a layoff due to establishment closure, a layoff due to economic reasons, a layoff for other reasons, early retirement, regular retirement, or for other reasons. The three outcome variables that we consider in (b) are indicators that equal one if a respondent's last employment spell ended between the ages of 50 and 54 and was a quit and was either (i) due to personal or family circumstances, (ii) sickness or disability, or (iii) other reasons. Each estimate stems from a separate regression based on 180,137 observations. As a baseline for (a), the values in the control region (among workers from REBP-eligible cohorts) are Quit (amicable or not): 0.00697; Layoff (closure): 0.00346; Layoff (economic reason): 0.0036; Layoff (other): 0.00275; Early Retirement: 0.0350; Regular Retirement: 0.0126; Other: 0.0383. For (b), control region values are Personal or family circumstances: 0.00182; Sickness or disability: 0.00389; Other reasons: 0.00126. The red vertical lines indicate 95\% confidence intervals based on standard errors clustered at the administrative region level. Using the Austrian Social Security Dataset, Panels (c) and (d) plots an indicator for those who do not take up UI until at least 28 days (the waiting period for quitters to receive UI) after the end of any of their employment spells between the ages of 50 and 55 . Note that there could be quitters who decide never to receive UI (see Section B.1) or who find re-employment before the end of the 28-day window. 


\section{Complier Characteristics in Difference-in-Differences IV Settings}

\section{C.1 Methodology}

Here, we provide the detailed, stand-alone methodological guide deriving and formalizing complier analysis in difference-in-differences contexts. For illustrative purposes, we follow the example of a program implemented across different regions and cohorts. To our knowledge, ours is the first characterization of sufficient conditions for complier analysis in difference-in-differences contexts. We complement recent advances to extend instrumental variable approaches to the differencein-differences framework (De Chaisemartin and D'Haultfoeuille, 2018: Hudson et al., 2017)) and build on the proof in Abadie (2003).

Setup We set up a potential outcomes framework for the difference-in-differences model (1) estimating the effect of REBP on separations among workers holding a job in pre-REBP in 1988, with fixed effects for region $r$ and cohort $c . D \in\{0,1\}$ indicates whether a given worker separates from their initial, pre-reform job by the end of the reform period (whether she was treated or not). $Z \in\{0,1\}$ captures whether workers are REBP-eligible (1) or not (0). $D_{0}$ and $D_{1}$ denote the potential values of $D$ for $Z=0$ and $Z=1\left(D=Z D_{1}+(1-Z) D_{0}\right)$. REBP varied by region $r \in\left\{r_{0}, r_{1}\right\}$ and cohort $c \in\left\{c_{0}, c_{1}\right\} . r_{0}$ is the control region and $r_{1}$ is the REBP region. $c_{0}\left(c_{1}\right)$ denotes ineligible (eligible) cohorts. We thus have that $Z=1$ for $\left(r_{1}, c_{1}\right)$ only, and $Z=0$ for other combinations $\left(r_{1}, c_{0}\right),\left(r_{0}, c_{0}\right)$ and $\left(r_{0}, c_{1}\right)$. Always-Separators have potential outcomes $\left(D_{0}=1, D_{1}=1\right)$ and share $\pi^{A S}$. Never-Separators have potential outcomes $\left(D_{0}=0, D_{1}=0\right)$ and share $\pi^{N S}$. Compliers - marginal jobs - have potential outcomes $\left(D_{0}=0, D_{1}=1\right)$ and share $\pi^{C}$. Even though individual compliers cannot be identified, we can identify the characteristics of compliers, i.e. $\mathbb{E}\left[x \mid r, c, D_{1}=1, D_{0}=0\right]$.

Identification Assumptions We make the following four assumptions to estimate complier characteristics in the difference-in-differences design. The assumptions are mostly common ones in an instrumental variables setup. For extending complier analysis to difference-in-differences settings, we additionally make an independence assumption for characteristics and assume additive separability for attributes.

A.1: First Stage For all $r \in\left(r_{0}, r_{1}\right)$ and $c \in\left(c_{0}, c_{1}\right), P\left(D_{1}=1 \mid r, c\right)>P\left(D_{0}=1 \mid r, c\right)$.

Intuitively, Assumption 1 implies that more separations take place under the reform and ensures the existence of compliers.

\section{A.2: Monotonicity $D_{1}-D_{0} \geq 0$.}

Assumption 2 rules out defiers, i.e. individuals that would separate if benefits are not extended but would not separate if unemployment benefits are more generous.

A.3: Independence $\left(D_{0}, D_{1}, x\right) \perp Z \mid(r, c)$.

The independence assumption posits that conditional on $r$ and $c$, the instrument $Z$ is orthogonal to potential outcomes $D_{0}$, and $D_{1}$ and, extending the usual assumptions in instrumental variables settings, also to attributes $x$.

A.4: Additive Separability For all $d, d^{\prime} \in\{0,1\}$, $P\left(D_{0}=d, D_{1}=d^{\prime} \mid c_{1}, r\right)-P\left(D_{0}=d, D_{1}=d^{\prime} \mid c_{0}, r\right)$ and $\mathbb{E}\left[x \mid c_{1}, r, D_{0}=d, D_{1}=d^{\prime}\right]-$ $\mathbb{E}\left[x \mid c_{0}, r, D_{0}=d, D_{1}=d^{\prime}\right]$ do not depend on $r$. 
The additive separability assumption for characteristics $x$ is analogous to assumptions for parallel trends or additive separability in recent work extending the instrumental variables setup to difference-in-differences settings (De Chaisemartin and D'Haultfoeuille (2018), Hudson et al. (2017)). A testable implication of Assumption 4 that we bring to the data is that $\mathbb{E}\left[x \mid c_{1}, r\right]-\mathbb{E}\left[x \mid c_{0}, r\right]$ does not depend on $r$. This is equivalent to saying that the between-cohort differences in the job attribute distribution are the same across regions, specifically among marginal jobs.

Identifying Characteristics Since $Z$ does not vary conditional on region and cohort, we only observe either $Z=0$, or $Z=1$, for a given region and cohort cell. Under the first stage and monotonicity assumption, the expected value of complier characteristics can be represented as a function of observable quantities for all region/cohort combinations.

We can rewrite the conditional expectation of individuals with $D_{1}=1$ in region $r$ and from cohort $c$ as follows:

$$
\begin{aligned}
\mathbb{E}\left[x \mid D_{1}=1, r, c\right]= & \mathbb{E}\left[x \mid r, c, D_{1}=1, D_{0}=1\right] \cdot P\left(D_{0}=1 \mid r, c, D_{1}=1\right) \\
& +\mathbb{E}\left[x \mid r, c, D_{1}=1, D_{0}=0\right] \cdot P\left(D_{0}=0 \mid r, c, D_{1}=1\right) .
\end{aligned}
$$

Rearranging yields

$$
\mathbb{E}\left[x \mid r, c, D_{1}=1, D_{0}=0\right]=\begin{gathered}
\frac{1}{P\left(D_{0}=0 \mid r, c, D_{1}=1\right)} \cdot \mathbb{E}\left[x \mid r, c, D_{1}=1\right] \\
-\frac{P\left(D_{0}=1 \mid r, c, D_{1}=1\right)}{P\left(D_{0}=0 \mid r, c, D_{1}=1\right)} \mathbb{E}\left[x \mid r, c, D_{1}=1, D_{0}=1\right] .
\end{gathered}
$$

By monotonicity $\left(D_{1}-D_{0} \geq 0\right)$, we have that $\mathbb{E}\left[x \mid r, c, D_{1}=1, D_{0}=1\right]=\mathbb{E}\left[x \mid r, c, D_{0}=1\right]$, which implies:

$$
\begin{aligned}
\mathbb{E}\left[x \mid r, c, D_{1}=1, D_{0}=0\right]= & \frac{1}{P\left(D_{0}=0 \mid r, c, D_{1}=1\right)} \cdot \mathbb{E}\left[x \mid r, c, D_{1}=1\right] \\
& -\frac{P\left(D_{0}=1 \mid r, c, D_{1}=1\right)}{P\left(D_{0}=0 \mid r, c, D_{1}=1\right)} \mathbb{E}\left[x \mid r, c, D_{0}=1\right] .
\end{aligned}
$$

Using the definition of conditional probabilities, $P\left(D_{0}=1 \mid r, c, D_{1}=1\right)=\frac{P\left(D_{0}=1, D_{1}=1 \mid r, c\right)}{P(D=1 \mid r, c)}$ and $P\left(D_{0}=0 \mid r, c, D_{1}=1\right)=\frac{P\left(D=0, D_{1}=1 \mid r, c\right)}{P\left(D_{1}=1 \mid r, c\right)}$. Define the (conditional on region and cohort) probability of always-separators as $\pi_{r c}^{A}=P\left(D_{0}=1, D_{1}=1 \mid r, c\right)$, of never-separators as $\pi_{r c}^{N}=P\left(D_{0}=0, D_{1}=\right.$ $0 \mid r, c)$ and by monotonicity of compliers as $\pi_{r c}^{C}=P\left(D_{0}=0, D_{1}=1 \mid r, c\right)=1-\pi_{r c}^{A}-\pi_{r c}^{N}$. The conditional expectation term above can then be expressed as follows:

$$
\begin{aligned}
\mathbb{E}\left[x \mid r, c, D_{1}=1, D_{0}=0\right]= & \frac{\pi_{r c}^{C}+\pi_{r c}^{A}}{\pi_{r c}^{C}} \cdot \mathbb{E}\left[x \mid r, c, D_{1}=1\right] \\
& -\frac{\pi_{r c}^{A}}{\pi_{r c}^{C}} \mathbb{E}\left[x \mid r, c, D_{0}=1\right] .
\end{aligned}
$$

Equation (A6) shows that complier characteristics are identified in a difference-in-difference IV setting. We can construct sample analogues to each of the terms on the right-hand side as follows by drawing on the independence and additive separability assumption. The following exposition follows the case of calculating characteristics conditional on $(r, c)=\left(r_{1}, c_{1}\right)$. By independence, we have that:

$$
\mathbb{E}\left[x \mid r_{1}, c_{1}, D_{1}=1\right]=\mathbb{E}\left[x \mid r_{1}, c_{1}, D=1, Z=1\right] .
$$


By independence and additive separability in $x$, we have:

$$
\begin{aligned}
\mathbb{E}\left[x \mid r_{1}, c_{1}, D_{0}=1\right] & =\mathbb{E}\left[x \mid r_{1}, c_{0}, D_{0}=1\right]+\left(\mathbb{E}\left[x \mid r_{0}, c_{1}, D_{0}=1\right]-\mathbb{E}\left[x \mid r_{0}, c_{0}, D_{0}=1\right]\right) \\
& =\mathbb{E}\left[x \mid r_{1}, c_{0}, D=1, Z=0\right] \\
& +\mathbb{E}\left[x \mid r_{0}, c_{1}, D=1, Z=0\right] \\
& -\mathbb{E}\left[x \mid r_{0}, c_{0}, D=1, Z=0\right] .
\end{aligned}
$$

Sample analogues exist for each of the right-hand side terms in (A7) and (A8):

$$
\begin{aligned}
& \mathbb{E}\left[x \mid r_{1}, c_{1}, D=1, Z=1\right]=\frac{1}{N_{r_{1}, c_{1}}} \sum_{i \in\left(r_{1}, c_{1}\right)} x_{i} D_{i}, \\
& \mathbb{E}\left[x \mid r_{1}, c_{0}, D=1, Z=0\right]=\frac{1}{N_{r_{1}, c_{0}}} \sum_{i \in\left(r_{1}, c_{0}\right)} x_{i} D_{i}, \\
& \mathbb{E}\left[x \mid r_{0}, c_{1}, D=1, Z=0\right]=\frac{1}{N_{r_{0}, c_{1}}} \sum_{i \in\left(r_{0}, c_{1}\right)} x_{i} D_{i}, \\
& \mathbb{E}\left[x \mid r_{0}, c_{0}, D=1, Z=0\right]=\frac{1}{N_{r_{0}, c_{0}}} \sum_{i \in\left(r_{0}, c_{0}\right)} x_{i} D_{i},
\end{aligned}
$$

where $N_{r_{1}, c_{1}}$ is the number of observations in $\left(r_{1}, c_{1}\right)$ and so forth.

For the conditional probabilities in $\mathrm{A} 6$ note that (using independence and the additive separability in $D$ assumption):

$$
\begin{aligned}
\pi_{r c}^{A}=P\left(D_{0}=1, D_{1}=1 \mid r_{1}, c_{1}\right) & =P\left(D_{0}=1 \mid r_{1}, c_{1}\right) \\
& =P\left(D_{0}=1 \mid r_{1}, c_{0}\right)+P\left(D_{0}=1 \mid r_{0}, c_{1}\right)-P\left(D_{0}=1 \mid r_{0}, c_{0}\right) \\
& =E\left(D \mid Z=0, r_{1}, c_{0}\right)+E\left(D \mid Z=0, r_{0}, c_{1}\right)-E\left(D \mid Z=0, r_{0}, c_{0}\right) \\
\pi_{r c}^{N}=P\left(D_{0}=0, D_{1}=0 \mid r_{1}, c_{1}\right) & =P\left(D_{1}=0 \mid r_{1}, c_{1}\right) \\
& =P\left(D=0 \mid Z=1, r_{1}, c_{1}\right) \\
& =1-E\left(D \mid Z=1, r_{1}, c_{1}\right) .
\end{aligned}
$$

These quantities can be estimated in the regression:

$$
D_{i r c}=\beta+\phi_{r}+\psi_{c}+v Z_{r c}+\chi_{i r c}
$$

The sample estimators are then given by $\pi_{r c}^{A}=\hat{\beta}+\hat{\phi}_{r}+\hat{\psi}_{c}, \pi_{r c}^{N}=1-\hat{\beta}-\hat{\phi}_{r}-\hat{\psi}_{c}-\hat{v}$ and $\pi_{r c}^{C}=1-\pi_{r c}^{N}-\pi_{r c}^{A}=\hat{v}$. All objects on the right-hand side of A6 thus have estimable sample counterparts.

Extensions Under additional assumptions, we can alternatively estimate the conditional expectations in (A6) in a regression framework. Specifically, if trends in $x$ are the same for alwaysseparators, always-separators and compliers, never-separators, and never-separators and compliers, the conditional expectations of characteristics can be estimated from the regression below:

$$
x_{i r c}=\alpha+\kappa_{r}+\lambda_{c}+\omega D_{i r c}+\gamma Z_{r c}+\varphi D_{i r c} \times Z_{r c}+\epsilon_{i r c} .
$$


This regression implies common trends across the four identified groups since the values of $D$ and $Z$ do not affect the trends $\kappa, \lambda$. The sample estimators are $\mathbb{E}\left[x \mid r, c, D_{1}=1\right]=\hat{\alpha}+\hat{\kappa}_{r}+\hat{\lambda}_{c}+\hat{\omega}+\hat{\gamma}+\hat{\varphi}$, and $\mathbb{E}\left[x \mid r, c, D_{0}=1\right]=\hat{\alpha}+\hat{\kappa}_{r}+\hat{\lambda_{c}}+\hat{\omega}$.

Under slightly weaker assumptions, not requiring us to assume parallel trends for neverseparators and compliers, we can use the following regression to estimate the required conditional expectations in Equation (A6) by interacting the trend variables with $D$ so that we can estimate separate trends for (1) always-separators and (2) never-separators and compliers:

$$
x_{i r c}=\alpha+\kappa_{r}+\lambda_{c}+\omega D_{i r c}+\tilde{\kappa}_{r} \times D_{i r c}+\tilde{\lambda}_{c} \times D_{i r c}+\gamma Z_{r c}+\varphi D_{i r c} \times Z_{r c}+\epsilon_{i r c}
$$

We then have $\mathbb{E}\left[x \mid r, c, D_{0}=1\right]=\alpha+\kappa_{r}+\lambda_{c}+\omega+\tilde{\kappa_{r}}+\tilde{\lambda_{c}}$ and $\mathbb{E}\left[x \mid r, c, D_{1}=1\right]=\alpha+\kappa_{r}+\lambda_{c}+$ $\omega+\tilde{\kappa_{r}}+\tilde{\lambda_{c}}+\gamma+\varphi$. Our approach can also be extended to estimate complier characteristics in the $Z=0$ cells if one of the following assumptions holds:

Assumption 5 (a). Cohort and region trends are the same for always-separators and compliers, or

Assumption 5 (b). Cohort and region trends are the same for always-separators and never-separators and either the proportion of compliers or never-separators is constant across cohort and region.

\section{C.2 Estimation Procedure for Complier Characteristics}

We describe the non-parametric bootstrapping procedure for inference. We use bootstrap samples of all employed men in 1988q2 working in either the non-REBP region or the region where REBP was abolished in 1993. As in the DiD specifications (Tables 2 through 3), we take samples by random clusters of administrative region $\sqrt{63}$ Specifically, we take blocks of the NUTS 3 designations for Austria, which consist of labor market districts (Bezirke) within the Austrian states (Bundesländer).

For each of the 1,000 random samplings of administrative regions, we

1. Estimmate the proportion of always-separators, never-separators, and compliers using Equations (A14) and (A15).

2. Estimate the average of each outcome among compliers, always-separators, and neverseparators by calculating the relevant conditional means.

3. Estimate regional and cohort averages to investigate parallel trends (see Table A.1). Specifically, for each outcome $Y_{i}$, we run the DiD specification

$$
Y_{i}=\beta+\phi_{r}+\psi_{c}+v Z_{r c}+\chi_{i r c}
$$

where every individual $i$ is in region $r$ (REBP vs. non-REBP) and cohort $c$ (REBP-eligible vs. ineligible). Then,

- For column (1) in Table A.1, the difference between the eligible and ineligible cohorts in the non-REBP region is $\hat{\psi}_{c}$.

- For column (2) in Table A.1 the cohort difference in the REBP region is $\hat{\psi}_{c}+\hat{v}$.

- For column (3) in Table A.1, the difference-in-differences is $\hat{v}$.

\footnotetext{
${ }^{63}$ In practice, we use the bsample command in Stata with the cluster option.
} 


\section{Investigate treatment effect heterogeneity.}

- Specifically, we create indicators for whether the individual is above or below the median value of each characteristic for continuous variables like earnings and an indicator for each value for binary characteristics like being in a blue-collar occupation.

- We run the DiD specification in Equation (1) separately for each group ${ }^{64}$

- We keep the two estimates of $\hat{v}$ and also take differences for the final column of Appendix Table A.2.

The final output is 1,000 estimates of each value of interest, one from each random sample of districts. The reported standard errors are the standard deviation of these 1,000 estimates. The reported point estimates are the same specifications run on the full sample.

\section{C.3 Detailed Discussion: Results of Complier Analysis}

Here we discuss the results of the complier analysis, summarized in the main text in Section 3.2 and in Figure 4 and Appendix Tables A.1 (baseline differences) and A.2 (complier characteristics compared to always- and never-separators).

Blue vs. White Collar Occupations An analysis of the occupational structure of marginal jobs (for data reasons limited to a classification into blue- and white-collar occupations) reveals that $82.3 \%$ of marginal jobs are in blue-collar occupations while the share of blue-collar workers among both always- and never-separators are lower at $67.9 \%$ and $50.5 \%$, respectively. When analyzing treatment effect heterogeneity, we also find stronger effects of the REBP on separations among blue-collar workers.

We also, in unreported results, considered the share of marginal jobs across industries and found a concentration in manual labor-intensive sectors such as mining or manufacturing. Virtually no marginal jobs exist in high-skilled, white-collar sectors such as health or banking.

Worker Codetermination, Works Councils, and Establishment Size We investigate whether compliers are particularly likely to originate from establishments with stronger worker codetermination through the works council. To do so, we explore the size cutoffs at 5, 20, 100, and 1,000 employees. At each threshold, the codetermination rights of workers are strengthened 65 As described in Section 2, workers can form a works council starting at establishment with five or more employees. In establishments with more than 20 employees, employer-works council agreements can be formed to take older workers' interests into consideration. In establishments with more than 100 employees, the local employment agency needs to be notified before sizable layoffs of workers. Finally, the works council can appeal to an external arbitration committee, e.g., in case of layoffs, when employment crosses a 200-employee threshold.

Our results show clear differences between compliers and never-separators across establishment size thresholds. Specifically, marginal jobs are more likely than never-separators to come from very large establishments where works councils can appeal to external arbitration committees $(85.9 \%$ vs. $54.4 \%)$. This pattern is consistent with stronger codetermination through works councils lending formal institutional support to implicit contracts as described in Lazear (1979.

${ }^{64}$ Equivalently, we fully interact the DiD specification with the indicator.

${ }^{65}$ Of course, other attributes also vary with establishment size so our analysis of worker codetermination and establishment size does not definitively pin down only variation in codetermination. See Jäger and Schoefer (2019) for more evidence on the effects of codetermination in the related context of Germany. 
1981) and Bewley (2002) and potentially consistent with our alternative non-Coasean framework described in Section 5 .

Employment Growth: Industry and Establishment Level Marginal jobs stem from industries or establishments with stagnating or declining labor demand. This complier attribute represents industry and establishment employment growth rates in the pre-period from 1982 to 1987 66 The analysis reveals that marginal jobs stem from declining industries which had a negative growth rate of -0.4ppt in the pre-period while both always- and never-separators come from moderately growing industries with positive growth rates of around 4.4 and 2.5ppt in the same time frame. The pattern is more pronounced at the establishment level, where we find that compliers stem from establishments with a negative average growth of -17.2ppt while never-separators stem from establishments with positive growth of 10.2ppt. Relatedly, only $11 \%$ of marginal jobs stem from establishments with positive employment growth in the pre-period while the shares of both always- and never-separators in growing establishments are substantially higher at 37.3\% and $56.3 \%$, respectively. Mirroring the complier characteristics, we also find that employment growth at the industry and establishment level correlates negatively with the treatment effect on separations, documenting that the REBP led to more separations when industries or firms were in decline. Overall, the evidence documents robustly that marginal jobs occurred in sectors and establishments that were declining, consistent with a low or decreasing joint surplus, worker surplus (lower expected continuation value), or firm surplus (at a given wage). It is consistent with a narrative in which REBP managed to buffer and perhaps accelerate labor market adjustment to some degree (since the compliers are, by construction, still present in the peer employers in the control regions and cohorts).

Earnings Annual earnings of compliers and never-separators are statistically indistinguishable (EUR 37,700 vs EUR 37,920, CPI-adjusted to 2018). Similarly, the earnings of compliers and alwaysseparators are also not distinguishable at the 5\% level. Relatedly, we do not find statistically significant treatment effect heterogeneity by earnings, conditional on the other covariates, in our estimation of treatment effect heterogeneity.

We further analyze AKM-specifications (Abowd et al., 1999) in the pre-period before 1988 and find that compliers and never-separators have similar firm and worker effects, with the point evidence perhaps pointing towards lower worker effects compared to never-separators. We estimate the following AKM model:

$$
\ln w_{i t}=\alpha_{i}+\psi_{J(i, t)}+X_{i t}^{\prime} \phi+v_{i t},
$$

where $\alpha_{i}$ and $\psi_{J(i, t)}$ denote worker and establishment fixed effects. Control vector $X_{i t}$ comprises a third-order polynomial in age. Worker effects $\alpha_{i}$ can be interpreted as the permanent component of wages workers command irrespective of the particular employer. Establishment effects $\psi_{J(i, t)}$ capture the wage premium or discount a given employer pays to a worker controlling for the worker effect. The fixed effects are identified through worker moves across employers, in the largest connected set 67

Our analysis reveals that the point estimates for worker effects are 7 log points smaller among compliers compared to never-separators, although the difference is not statistically significant. The results are consistent with marginal matches having permanently lower earnings compared to their peers, although the imprecision of the estimate prohibits a strong interpretation. Marginal jobs and never-separators have indistinguishable establishment effects, with the point estimate for the

\footnotetext{
${ }^{66}$ This analysis is therefore restricted to those establishments we observe in the pre-REBP period.

${ }^{67}$ For estimation, we follow the procedure in Correia et al. (2016).
} 
difference being 2.7 log points (larger establishment effects among marginal jobs). The treatment effect heterogeneity analysis also reveals no statistically significant differences in treatment effects between matches with different establishment or worker effects. One interpretation is that the excess REBP separations stem from matches with similar firm surplus, since establishment fixed effects have been shown to correlate positively with measures of value added per worker (see Card et al. 2018).

Tenure The point estimate for tenure of workers in marginal jobs is slightly higher compared to the whole sample, although the difference is not statistically significant (same for treatment effect heterogeneity). The complier mean for tenure in 1988 is 13.48 years compared to 11.61 years for never-separators. Tenure is a particularly ambiguous attribute as it may be associated with distinctly negative firm surplus but positive worker surplus (Lazear, 1979, 1981; Frimmel et al. 2018), but alternatively positive joint surplus due to ex ante investments (Oi. 1962) or match quality due to selection mechanism (Cahuc et al., 2006: Hagedorn and Manovskii, 2013;: Bagger et al., 2014).

Sickness and Disability Measures of worker sickness and disability are interesting complier attributes because they may capture disutility of labor or lower productivity. Since we analyze a sample of workers employed at the onset of the reform, we cannot focus on sick leave or disability spells at that time. Instead, we focus on sick leave and disability rates at the 4-digit industry level in the pre-reform period. We exploit the ASSD administrative registration of these spells. For both indicators, sick leave and disability, as well as for the combined rate of the two, we find that compliers come from industries with higher indicators of morbidity among older workers. For example, compliers' industries have a $0.8 \mathrm{ppt}$ higher share of workers on sick leave or disability compared to never-separators, about $50 \%$ of a standard deviation 68 Similarly, these industries exhibited stronger treatment effects. Overall, these results suggest that REBP dissolved matches in which the disutility of working increases or worker productivity decreases with age, consistent with low and shrinking (worker) surplus. ${ }^{69}$

Long-Term Nonemployment Risk Finally, we consider an indicator for risk of long-term nonemployment. We do so by regressing an indicator for experiencing a nonemployment spell longer than one year on a set of predictors among the sample of workers in the pre-reform period from 1982 to 1987. The pre-separation variables we include for the prediction are fixed effects for 15 large industries, an indicator for being in a white-collar occupation, the local unemployment rate, and third-degree polynomials of tenure in current job and experience over the last 25 years. We then analyze the predicted risk of a long-term nonemployment spell as a complier attribute for our sample of pre-REBP 1988 jobs.

Compared to the never-separators, compliers have a 7.4 ppt higher risk of experiencing an nonemployment spell longer than one year based on our prediction. The difference is statistically significant. The point estimates for treatment effect heterogeneity along this dimension point in a similar direction although are less precisely estimated. Overall, the evidence suggests that the REBP reform selected those workers who had a higher risk of experiencing long-term nonemployment after a separation even in the absence of the reform. This perspective is consistent with our finding

\footnotetext{
${ }^{68}$ These proxies are powerful because for workers younger than 55 , a disability spell indicates severe health problems as disability insurance formally requires medical impairment to reduce the capacity to work by at least 50 percent in any occupation. The highest incidence of sickness and disability can be found in mining, construction, and manufacturing, sectors dominated by blue-collar labor, which also showed a high share of marginal jobs.

${ }^{69} \mathrm{~A}$ caveat to this interpretation arises from the potential positive selection implied by the fact that our sample considers workers employed at that age in these industries at the onset of the reform.
} 
that most of the excess REBP separators went into long-term nonemployment as documented in Section 3 . Moreover, the workers that separated from their 1988 job in response to REBP evidently were unlikely to find, or seek, reemployment, consistent with the workers having low surplus with respect to nonemployment, as in our framework in Sections 5 and 6 


\section{Variable Construction}

We describe the construction of each outcome variable presented in the paper. In the descriptions below, status refers to a variable in the ASSD aggregating hundreds of administrative designations into 12 labor market statuses (Zweimüller et al. 2009). We classify self-employment (status $==6$ ) and mini-jobs (status $==10$ ) as employment.

\section{Separation}

- Create an indicator if equal to one, between two periods (e.g., 1988q2 and 1993q3), the worker is observed in the same establishment.

- If not, the worker is separated

\section{Separation into Nonemployment}

- Create an indicator equal to one if the worker separated as defined above and had no other employer between 1988q2 and 1993q3.

\section{Separation into New Job}

- Create an indicator equal to one if the worker separated as defined above and had another employer (establishment) in the interim.

\section{Unemployment (Months)}

- Between two periods (e.g., 1988q2 and 1993q3), count the number of quarters where the worker is observed on UI or UA (status $=1$ ).

- Multiply the quarter count by 3 to get a monthly count for tractability.

\section{Continuous Employment (Months)}

- Between two periods (e.g., 1988q2 and 1993q3), count the number of quarters where the worker is employed in the same establishment as the quarter before.

- Stop counting when the worker is observed either employed in a new establishment or with another labor market status.

\section{Quit (Indicator)}

- Out of the original labor market spell data from the ASSD, count the number of days between the end of an employment spell (status $=3$ ) and the beginning of an unemployment spell (status $=1$ ).

- For the yearly analysis, isolate employment spells that end during the REBP period (1988q2 through 1993q3).

- For the age analysis, isolate employment spells that end while the worker is between 50 and 55 years old.

- If the beginning of the unemployment spell occurs 28 days or more after the end of any employment spell during this range, the worker is considered to have quit.

\section{Local Unemployment Rate}

- Take the snapshot of men in the ASSD from 1988q2 (May 15, 1988). 
- Assign each worker a municipality (Gemeinde) by the location of the establishment at which they work.

- Count the number on UI (status =1) in each municipality.

- Count the number of workers not on any pension, i.e. neither on disability (e.g., Berufsunfähigkeitspension) nor retirement pensions.

\section{Local Unemp. Rate (50+)}

- Do the same as for unemployment rates, but restrict to the sample of workers who are 50 years old or older in $1988 \mathrm{q} 2$.

\section{Establishment Size}

- For every establishment, count the total number of men of any age employed in 1988q2.

\section{Employment Growth at Establishment}

- In Table 1 and the complier analysis, this counts the percent difference between the number of men (no age restriction) employed in the establishment in 1983q2 and 1988q2.

- In Figure 9, this is the percent difference between the number of men born in 1933 or later (to avoid retirements) and employed in each establishment in 1994q1 and Q1 of each following year.

\section{1(Growing Establishment)}

- In all cases, this is an indicator for whether the establishment employment growth variable calculated above is positive.

\section{Employment Growth at Industry}

- This counts the percent difference between the number of men (no age restriction) employed in one of 15 industries in 1983q2 and 1988q2.

\section{Establishment Earnings Fixed Effects (AKM)}

- Begin with the sample of all workers in May 1977 through 1987.

- The earnings value is the total annual earnings in 2002 EUR from the establishment at which the worker is employed on June 15 of each year.

- Winsorize the earnings value to the 5th and 95th percentile by year and take the logarithm.

- Regress log earnings on a third-degree polynomial of age and fixed effects for each worker and establishment using the procedure to estimate high-dimensional fixed effects outlined in Correia et al. (2016).

- Assign to each worker the establishment fixed effect from the establishment at which they work in 1988q2.

\section{Worker Earnings Fixed Effects (AKM)}

- Use the worker fixed effects estimated in the proecedure outlined to estimate establishment fixed effects. 


\section{Share on Sick Leave/Disability in Industry}

- Begin with the sample of men age 50 to 55 in any quarter of years 1977 through 1988.

- If workers are nonemployed in a given quarter, assign the establishment identifier of their last employe, i.e. the establishment identifier of the last establishment where status $=3$.

- These assigned establishments are used to assign industry.

- By four-digit NACE industry level, calculate the share of workers on sick leave (status $=2$ ) and receiving disability payments (status $=5$ ) as a fraction of all workers assigned to that industry across all periods.

\section{Long Spell Duration Risk}

- Begin with the sample of all employed men in 1982 and track them through 1987.

- Count the length of nonemployment spells.

- Isolate the maximum length of a nonempmloyment spell for each worker.

- Create an indicator for whether the maximum length of the nonemployment spell for each worker is greater than 1 year (4 quarters).

- Regress this indicator on fixed effects for 15 large industries, an indicator for being in a white-collar occupation, the local unemployment rate (see above), and third-degree polynomials of tenure in current job and experience over the last 25 years.

- Predict the outcome, i.e. risk of long nonemployment duration, for every worker in the REBP sample using these estimates. 


\section{E Additional Tables}

Table A.1: Complier Characteristics: Balance Check

\begin{tabular}{|c|c|c|c|}
\hline & Non-REBP Region & REBP Region & Difference \\
\hline \multirow[t]{2}{*}{ Blue Collar } & 0.0180 & 0.0270 & 0.00900 \\
\hline & $(0.005)$ & $(0.005)$ & $(0.008)$ \\
\hline \multirow[t]{2}{*}{ Emp. Growth Industry } & -0.00500 & -0.00700 & -0.00200 \\
\hline & $(0.001)$ & $(0.001)$ & $(0.001)$ \\
\hline \multirow[t]{2}{*}{ Emp. Growth at Establishment } & -0.0310 & -0.0540 & -0.0220 \\
\hline & $(0.002)$ & $(0.006)$ & $(0.007)$ \\
\hline \multirow[t]{2}{*}{ 1(Growing Establishment) } & -0.0220 & -0.0480 & -0.0260 \\
\hline & $(0.004)$ & $(0.010)$ & $(0.011)$ \\
\hline \multirow[t]{2}{*}{ Annual Earnings (1,000 EUR) } & 0.313 & 0.401 & 0.0880 \\
\hline & $(0.071)$ & $(0.130)$ & $(0.136)$ \\
\hline \multirow[t]{2}{*}{ Establishment Earnings Fixed Effect (AKM) } & 0.0140 & 0.0110 & -0.00300 \\
\hline & $(0.002)$ & $(0.003)$ & $(0.004)$ \\
\hline \multirow[t]{2}{*}{ Worker Earnings Fixed Effect (AKM) } & -0.0310 & -0.0330 & -0.00200 \\
\hline & $(0.003)$ & $(0.004)$ & $(0.005)$ \\
\hline \multirow[t]{2}{*}{ Tenure } & 1.276 & 1.502 & 0.226 \\
\hline & $(0.078)$ & $(0.094)$ & $(0.107)$ \\
\hline \multirow[t]{2}{*}{ Share on Sick Leave in Industry $\times 100$} & -0.00200 & 0.00300 & 0.00500 \\
\hline & $(0.002)$ & $(0.002)$ & $(0.003)$ \\
\hline \multirow[t]{2}{*}{ Share on Disability in Industry $\times 100$} & 0.0540 & 0.124 & 0.0700 \\
\hline & $(0.022)$ & $(0.027)$ & $(0.035)$ \\
\hline \multirow[t]{2}{*}{ Share on Sick Leave/Disability in Industry $\times 100$} & 0.0520 & 0.129 & 0.0770 \\
\hline & $(0.024)$ & $(0.029)$ & $(0.038)$ \\
\hline \multirow[t]{2}{*}{ Long Nonemployment Spell Duration Risk } & 0.0710 & 0.0760 & 0.00500 \\
\hline & $(0.001)$ & $(0.002)$ & $(0.002)$ \\
\hline \multirow[t]{2}{*}{ Establishment Size: 5 or Less } & -0.00700 & -0.00800 & -0.00100 \\
\hline & $(0.001)$ & $(0.003)$ & $(0.003)$ \\
\hline \multirow[t]{2}{*}{ Establishment Size: 6 and 20} & -0.00700 & -0.0120 & -0.00500 \\
\hline & $(0.002)$ & $(0.002)$ & $(0.003)$ \\
\hline \multirow[t]{2}{*}{ Establishment Size: 21 and 100} & -0.00200 & -0.00800 & -0.00600 \\
\hline & $(0.006)$ & $(0.008)$ & $(0.008)$ \\
\hline \multirow[t]{2}{*}{ Establishment Size: 101 and 200} & 0.00200 & 0.00300 & 0.00100 \\
\hline & $(0.002)$ & $(0.005)$ & $(0.006)$ \\
\hline \multirow[t]{2}{*}{ Establishment Size: 201 or Greater } & 0.0140 & 0.0260 & 0.0120 \\
\hline & $(0.007)$ & $(0.011)$ & $(0.012)$ \\
\hline
\end{tabular}

Note: This table reports the mean difference for the characteristics reported in the left column between REBP-eligible and -ineligible cohorts in the non-REBP Region (column 1) vs. the REBP Region (column 2). Column (3) reports the difference between column (2) and (1). For an overview of the methodology underlying the decomposition into the groups and a detailed discussion of the results, see Appendix $\mathrm{C}$ and Appendix $\mathrm{D}$ for details on how the variables are constructed. For each of the variables and groups, the table reports means as well as standard errors (in parentheses) based on 1,000 bootstrap replications blocked at the administrative region level. Given the small percentage-point differences, the industry share variables are multiplied by 100 for legibility. Earnings are reported in thousands of 2018 Euros. 
Table A.2: Complier Characteristics and Heterogeneous Treatment Effects: Results

\begin{tabular}{|c|c|c|c|c|c|c|c|}
\hline & Complier & Never-Sep & Diff. (C-N) & Always-Sep & Diff. (C-A) & SD & $\begin{array}{l}\text { Interaction w/ } \\
\text { REBP Eligibility }\end{array}$ \\
\hline \multirow[t]{2}{*}{ Blue Collar } & 0.823 & 0.505 & 0.318 & 0.679 & 0.144 & 0.499 & 0.0630 \\
\hline & $(0.054)$ & $(0.053)$ & $(0.056)$ & $(0.040)$ & $(0.061)$ & & $(0.022)$ \\
\hline \multirow[t]{2}{*}{ Emp. Growth Industry } & -0.00400 & 0.0440 & -0.0480 & 0.0250 & -0.0290 & 0.0670 & -0.0990 \\
\hline & $(0.013)$ & $(0.007)$ & $(0.012)$ & $(0.005)$ & $(0.014)$ & & $(0.038)$ \\
\hline \multirow[t]{2}{*}{ Emp. Growth at Establishment } & -0.172 & 0.102 & -0.274 & 0.0100 & -0.182 & 0.303 & -0.0760 \\
\hline & $(0.079)$ & $(0.014)$ & $(0.073)$ & $(0.031)$ & $(0.083)$ & & $(0.037)$ \\
\hline \multirow[t]{2}{*}{ 1(Growing Establishment) } & 0.111 & 0.563 & -0.452 & 0.373 & -0.262 & 0.499 & -0.0840 \\
\hline & $(0.117)$ & $(0.033)$ & $(0.103)$ & $(0.032)$ & $(0.117)$ & & $(0.037)$ \\
\hline \multirow[t]{2}{*}{ Annual Earnings (1,000 EUR) } & 37.70 & 37.92 & -0.216 & 35.41 & 2.294 & 9.869 & 0.0200 \\
\hline & $(1.640)$ & $(1.077)$ & $(1.464)$ & $(1.091)$ & $(1.773)$ & & $(0.026)$ \\
\hline \multirow[t]{2}{*}{ Establishment Earnings Fixed Effect (AKM) } & 0.0380 & 0.0110 & 0.0270 & 0.0350 & 0.00300 & 0.224 & -0.00400 \\
\hline & $(0.038)$ & $(0.011)$ & $(0.039)$ & $(0.011)$ & $(0.041)$ & & $(0.027)$ \\
\hline \multirow[t]{2}{*}{ Worker Earnings Fixed Effect (AKM) } & 0.0710 & 0.141 & -0.0700 & 0.0460 & 0.0250 & 0.271 & -0.0120 \\
\hline & $(0.059)$ & $(0.021)$ & $(0.050)$ & $(0.020)$ & $(0.054)$ & & $(0.021)$ \\
\hline \multirow[t]{2}{*}{ Tenure } & 13.48 & 11.61 & 1.868 & 10.77 & 2.710 & 5.845 & 0.0350 \\
\hline & $(1.700)$ & $(0.578)$ & $(1.876)$ & $(0.784)$ & $(2.150)$ & & $(0.038)$ \\
\hline \multirow[t]{2}{*}{ Share on Sick Leave in Industry } & 0.00900 & 0.00800 & 0.00100 & 0.00800 & 0.00100 & 0.00200 & 0.0930 \\
\hline & $(0.000)$ & $(0.000)$ & $(0.000)$ & $(0.000)$ & $(0.000)$ & & $(0.071)$ \\
\hline \multirow[t]{2}{*}{ Share on Disability in Industry } & 0.0610 & 0.0540 & 0.00700 & 0.0650 & -0.00400 & 0.0130 & 0.0730 \\
\hline & $(0.005)$ & $(0.002)$ & $(0.004)$ & $(0.001)$ & $(0.006)$ & & $(0.038)$ \\
\hline \multirow[t]{2}{*}{ Share on Sick Leave/Disability in Industry } & 0.0700 & 0.0620 & 0.00800 & 0.0740 & -0.00400 & 0.0140 & 0.0580 \\
\hline & $(0.005)$ & $(0.002)$ & $(0.005)$ & $(0.001)$ & $(0.006)$ & & $(0.027)$ \\
\hline \multirow[t]{2}{*}{ Long Nonemployment Spell Duration Risk } & 0.434 & 0.360 & 0.0740 & 0.363 & 0.0700 & 0.127 & 0.0530 \\
\hline & $(0.030)$ & $(0.005)$ & $(0.029)$ & $(0.008)$ & $(0.034)$ & & $(0.045)$ \\
\hline \multirow[t]{2}{*}{ Establishment Size: 5 or Less } & 0.0980 & 0.0900 & 0.00800 & 0.0550 & 0.0420 & 0.281 & 0.00200 \\
\hline & $(0.063)$ & $(0.014)$ & $(0.063)$ & $(0.008)$ & $(0.063)$ & & $(0.030)$ \\
\hline \multirow[t]{2}{*}{ Establishment Size: 6 and 20} & 0.0190 & 0.0790 & -0.0600 & 0.0570 & -0.0380 & 0.295 & -0.0540 \\
\hline & $(0.033)$ & $(0.008)$ & $(0.034)$ & $(0.008)$ & $(0.036)$ & & $(0.032)$ \\
\hline \multirow[t]{2}{*}{ Establishment Size: 21 and 100} & -0.0210 & 0.232 & -0.253 & 0.425 & -0.446 & 0.447 & -0.110 \\
\hline & $(0.078)$ & $(0.018)$ & $(0.070)$ & $(0.036)$ & $(0.094)$ & & $(0.042)$ \\
\hline \multirow[t]{2}{*}{ Establishment Size: 101 and 200} & 0.0450 & 0.0550 & -0.0100 & 0.0640 & -0.0190 & 0.237 & -0.0140 \\
\hline & $(0.057)$ & $(0.007)$ & $(0.057)$ & $(0.022)$ & $(0.055)$ & & $(0.042)$ \\
\hline \multirow[t]{2}{*}{ Establishment Size: 201 or Greater } & 0.859 & 0.544 & 0.315 & 0.398 & 0.461 & 0.500 & 0.102 \\
\hline & $(0.144)$ & $(0.036)$ & $(0.139)$ & $(0.037)$ & $(0.146)$ & & $(0.051)$ \\
\hline
\end{tabular}

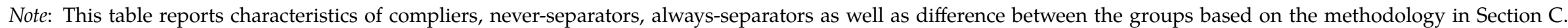

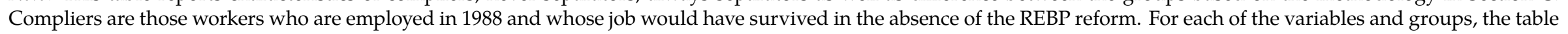

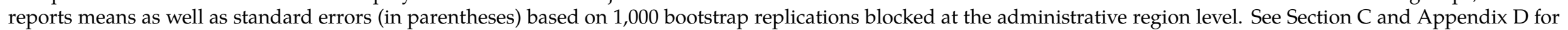

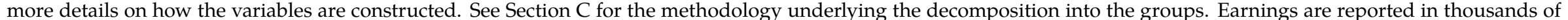
2018 Euros. 
Table A.3: Difference-in-Differences Effects of the REBP on Outcomes of Initially Employed Workers (Age 50 to 55)

\begin{tabular}{|c|c|c|c|c|c|}
\hline & $\begin{array}{c}(1) \\
\text { Separation }\end{array}$ & $\begin{array}{c}(2) \\
\text { Separation } \\
\text { Into Nonemployment }\end{array}$ & $\begin{array}{c}\text { (3) } \\
\text { Nonemployment } \\
\text { (Quarters) }\end{array}$ & $\begin{array}{c}(4) \\
\text { Unemp. (Benefits) } \\
\text { (Quarters) }\end{array}$ & $\begin{array}{l}\text { (5) } \\
\text { Cont. Empl. } \\
\text { (Quarters) }\end{array}$ \\
\hline REBP Region $\times$ Treated Cohort & $\begin{array}{c}0.124^{* * *} \\
(0.026)\end{array}$ & $\begin{array}{l}0.098^{* *} \\
(0.040)\end{array}$ & $\begin{array}{l}1.027^{* *} \\
(0.506)\end{array}$ & $\begin{array}{c}0.745 \\
(0.560)\end{array}$ & $\begin{array}{c}-1.147^{* * *} \\
(0.328)\end{array}$ \\
\hline REBP Region & $\begin{array}{c}-0.035 \\
(0.031)\end{array}$ & $\begin{array}{l}0.022^{* *} \\
(0.010)\end{array}$ & $\begin{array}{c}0.129 \\
(0.302)\end{array}$ & $\begin{array}{c}0.129 \\
(0.238)\end{array}$ & $\begin{array}{c}0.559 \\
(0.527)\end{array}$ \\
\hline Treated Cohort & $\begin{array}{c}-0.070^{* * *} \\
(0.015)\end{array}$ & $\begin{array}{c}-0.006^{* *} \\
(0.002)\end{array}$ & $\begin{array}{l}-0.381 \\
(0.312)\end{array}$ & $\begin{array}{c}-0.249 \\
(0.181)\end{array}$ & $\begin{array}{l}1.174^{* *} \\
(0.581)\end{array}$ \\
\hline Constant & $\begin{array}{c}0.424^{* * *} \\
(0.080)\end{array}$ & $\begin{array}{c}0.130^{* * *} \\
(0.021)\end{array}$ & $\begin{array}{c}2.299^{* * *} \\
(0.822)\end{array}$ & $\begin{array}{l}1.117^{*} \\
(0.603)\end{array}$ & $\begin{array}{c}14.605^{* * *} \\
(1.638)\end{array}$ \\
\hline $\begin{array}{l}\text { Observations } \\
\text { Adjusted } R^{2} \\
\text { No of Clusters }\end{array}$ & $\begin{array}{c}378,693 \\
0.006 \\
100\end{array}$ & $\begin{array}{c}378,693 \\
0.014 \\
100\end{array}$ & $\begin{array}{c}378,693 \\
0.008 \\
100\end{array}$ & $\begin{array}{c}378,693 \\
0.011 \\
100\end{array}$ & $\begin{array}{c}378,693 \\
0.005 \\
100\end{array}$ \\
\hline
\end{tabular}

Note: The table reports results of the econometric specification in (1). REBP captures the effect of REBP-eligibility on the outcomes listed in columns (1) through (5) on a sample of workers employed in the quarter before turning 50. Separation denotes an indicator function that is 1 if a worker is not employed by their employer at age 49.75 by the quarter before they turn 55. Separation into Nonemployment denotes an indicator for Separation from the initial employer interacted with an indicator for not taking up employment with another employer by the quarter before turning 55. Nonemployment (Quarters), Unemployment (Benefits) (Quarters), and Continuous Employment (Quarters) denote the quarters of nonemployment, unemployment benefits, and continuous employment with the initial employer between age 50 and age 55. Standard errors clustered at the administrative region level are reported in parentheses. Levels of significance: ${ }^{*} 10 \%$, ${ }^{* *}$ $5 \%$, and ${ }^{* * *} 1 \%$ 
Table A.4: Difference-in-Differences Effects of the REBP on Outcomes of Survivors (1994 Through 1995)

\begin{tabular}{|c|c|c|c|c|c|}
\hline & $\begin{array}{c}(1) \\
\text { Separation }\end{array}$ & $\begin{array}{c}(2) \\
\text { Separation } \\
\text { Into Nonemployment }\end{array}$ & $\begin{array}{c}\text { (3) } \\
\text { Nonemployment } \\
\text { (Quarters) }\end{array}$ & $\begin{array}{c}(4) \\
\text { Unemp. (Benefits) } \\
\text { (Quarters) }\end{array}$ & $\begin{array}{c}\text { (5) } \\
\text { Cont. Empl. } \\
\text { (Quarters) }\end{array}$ \\
\hline REBP Region $\times$ Treated Cohort & $\begin{array}{l}0.008^{*} \\
(0.005)\end{array}$ & $\begin{array}{c}0.004 \\
(0.002)\end{array}$ & $\begin{array}{c}0.002 \\
(0.008)\end{array}$ & $\begin{array}{l}-0.022 \\
(0.014)\end{array}$ & $\begin{array}{r}-0.016 \\
(0.010)\end{array}$ \\
\hline REBP Region & $\begin{array}{c}-0.012 \\
(0.011)\end{array}$ & $\begin{array}{l}-0.001 \\
(0.007)\end{array}$ & $\begin{array}{l}-0.008 \\
(0.018)\end{array}$ & $\begin{array}{c}0.002 \\
(0.013)\end{array}$ & $\begin{array}{l}0.058^{* *} \\
(0.026)\end{array}$ \\
\hline Treated Cohort & $\begin{array}{c}0.072^{* * *} \\
(0.005)\end{array}$ & $\begin{array}{c}0.083^{* * *} \\
(0.001)\end{array}$ & $\begin{array}{c}0.198^{* * *} \\
(0.006)\end{array}$ & $\begin{array}{l}0.048^{* *} \\
(0.021)\end{array}$ & $\begin{array}{c}-0.181^{* * *} \\
(0.018)\end{array}$ \\
\hline Constant & $\begin{array}{c}0.093^{* * *} \\
(0.029)\end{array}$ & $\begin{array}{l}0.044^{* *} \\
(0.019)\end{array}$ & $\begin{array}{l}0.113^{* *} \\
(0.047)\end{array}$ & $\begin{array}{c}0.044 \\
(0.036)\end{array}$ & $\begin{array}{l}4.744^{* * *} \\
(0.069)\end{array}$ \\
\hline $\begin{array}{l}\text { Observations } \\
\text { Adjusted } R^{2} \\
\text { No of Clusters }\end{array}$ & $\begin{array}{c}207,785 \\
0.011 \\
99\end{array}$ & $\begin{array}{c}207,785 \\
0.020 \\
99\end{array}$ & $\begin{array}{c}207,785 \\
0.017 \\
99\end{array}$ & $\begin{array}{c}207,785 \\
0.003 \\
99\end{array}$ & $\begin{array}{c}207,785 \\
0.009 \\
99\end{array}$ \\
\hline
\end{tabular}

Note: See the note for Table 3 for details. 
Table A.5: Difference-in-Differences Effects of the REBP on Outcomes of Survivors (1994 Through 1997)

\begin{tabular}{|c|c|c|c|c|c|}
\hline & $\begin{array}{c}(1) \\
\text { Separation }\end{array}$ & $\begin{array}{c}(2) \\
\text { Separation } \\
\text { Into Nonemployment }\end{array}$ & $\begin{array}{c}\text { (3) } \\
\text { Nonemployment } \\
\text { (Quarters) }\end{array}$ & $\begin{array}{c}(4) \\
\text { Unemp. (Benefits) } \\
\text { (Quarters) }\end{array}$ & $\begin{array}{c}\text { (5) } \\
\text { Cont. Empl. } \\
\text { (Quarters) }\end{array}$ \\
\hline REBP Region $\times$ Treated Cohort & $\begin{array}{l}0.022^{* *} \\
(0.010)\end{array}$ & $\begin{array}{c}0.019 * * * \\
(0.007)\end{array}$ & $\begin{array}{c}0.076 \\
(0.055)\end{array}$ & $\begin{array}{c}-0.122 \\
(0.082)\end{array}$ & $\begin{array}{l}-0.120^{*} \\
(0.069)\end{array}$ \\
\hline REBP Region & $\begin{array}{l}-0.015 \\
(0.026)\end{array}$ & $\begin{array}{c}0.005 \\
(0.013)\end{array}$ & $\begin{array}{l}-0.005 \\
(0.113)\end{array}$ & $\begin{array}{c}0.010 \\
(0.083)\end{array}$ & $\begin{array}{c}0.172 \\
(0.184)\end{array}$ \\
\hline Treated Cohort & $\begin{array}{c}0.200^{* * *} \\
(0.014)\end{array}$ & $\begin{array}{c}0.240^{* * *} \\
(0.005)\end{array}$ & $\begin{array}{c}1.555^{* * *} \\
(0.014)\end{array}$ & $\begin{array}{c}0.227^{*} \\
(0.123)\end{array}$ & $\begin{array}{c}-1.319^{* * *} \\
(0.119)\end{array}$ \\
\hline Constant & $\begin{array}{c}0.220^{* * *} \\
(0.069)\end{array}$ & $\begin{array}{l}0.090^{* *} \\
(0.036)\end{array}$ & $\begin{array}{l}0.638^{* *} \\
(0.284)\end{array}$ & $\begin{array}{c}0.269 \\
(0.209)\end{array}$ & $\begin{array}{c}11.297^{* * *} \\
(0.509)\end{array}$ \\
\hline $\begin{array}{l}\text { Observations } \\
\text { Adjusted } R^{2} \\
\text { No of Clusters }\end{array}$ & $\begin{array}{c}207,785 \\
0.045 \\
99\end{array}$ & $\begin{array}{c}207,785 \\
0.079 \\
99\end{array}$ & $\begin{array}{c}207,785 \\
0.062 \\
99\end{array}$ & $\begin{array}{c}207,785 \\
0.006 \\
99\end{array}$ & $\begin{array}{c}207,785 \\
0.029 \\
99\end{array}$ \\
\hline
\end{tabular}

Note: See the note for Table 3 for details. 
Table A.6: Difference-in-Differences Effects of the REBP on Outcomes of Survivors (1994 Through 1998)

\begin{tabular}{|c|c|c|c|c|c|}
\hline & $\begin{array}{c}(1) \\
\text { Separation }\end{array}$ & $\begin{array}{c}(2) \\
\text { Separation } \\
\text { Into Nonemployment }\end{array}$ & $\begin{array}{c}\text { (3) } \\
\text { Nonemployment } \\
\text { (Quarters) }\end{array}$ & $\begin{array}{c}(4) \\
\text { Unemp. (Benefits) } \\
\text { (Quarters) }\end{array}$ & $\begin{array}{c}\text { (5) } \\
\text { Cont. Empl. } \\
\text { (Quarters) }\end{array}$ \\
\hline REBP Region $\times$ Treated Cohort & $\begin{array}{l}0.026^{* *} \\
(0.011)\end{array}$ & $\begin{array}{l}0.018^{*} \\
(0.009)\end{array}$ & $\begin{array}{c}0.145 \\
(0.096)\end{array}$ & $\begin{array}{l}-0.170 \\
(0.114)\end{array}$ & $\begin{array}{l}-0.224^{* *} \\
(0.110)\end{array}$ \\
\hline REBP Region & $\begin{array}{c}-0.022 \\
(0.029)\end{array}$ & $\begin{array}{c}0.008 \\
(0.014)\end{array}$ & $\begin{array}{l}-0.001 \\
(0.184)\end{array}$ & $\begin{array}{c}0.015 \\
(0.133)\end{array}$ & $\begin{array}{c}0.262 \\
(0.299)\end{array}$ \\
\hline Treated Cohort & $\begin{array}{c}0.241^{* * *} \\
(0.015)\end{array}$ & $\begin{array}{c}0.299 * * * \\
(0.011)\end{array}$ & $\begin{array}{c}2.651^{* * *} \\
(0.039)\end{array}$ & $\begin{array}{c}0.282^{*} \\
(0.169)\end{array}$ & $\begin{array}{c}-2.187^{* * *} \\
(0.194)\end{array}$ \\
\hline Constant & $\begin{array}{c}0.293^{* * *} \\
(0.080)\end{array}$ & $\begin{array}{c}0.122^{* * *} \\
(0.038)\end{array}$ & $\begin{array}{l}1.053^{* *} \\
(0.461)\end{array}$ & $\begin{array}{c}0.434 \\
(0.334)\end{array}$ & $\begin{array}{c}14.154^{* * *} \\
(0.837)\end{array}$ \\
\hline $\begin{array}{l}\text { Observations } \\
\text { Adjusted } R^{2} \\
\text { No of Clusters }\end{array}$ & $\begin{array}{c}207,785 \\
0.059 \\
99\end{array}$ & $\begin{array}{c}207,785 \\
0.104 \\
99\end{array}$ & $\begin{array}{c}207,785 \\
0.085 \\
99\end{array}$ & $\begin{array}{c}207,785 \\
0.005 \\
99\end{array}$ & $\begin{array}{c}207,785 \\
0.040 \\
99\end{array}$ \\
\hline
\end{tabular}

Note: See the note for Table 3 for details. 
Table A.7: Robustness to Retirement Dynamics (Dropping Cohorts Born Before 1938): 1994 Through 1996

\begin{tabular}{|c|c|c|c|c|c|}
\hline & $\begin{array}{c}(1) \\
\text { Separation }\end{array}$ & $\begin{array}{c}(2) \\
\text { Separation } \\
\text { Into Nonemployment }\end{array}$ & $\begin{array}{c}\text { (3) } \\
\text { Nonemployment } \\
\text { (Quarters) }\end{array}$ & $\begin{array}{c}(4) \\
\text { Unemp. (Benefits) } \\
\text { (Quarters) }\end{array}$ & $\begin{array}{c}\text { (5) } \\
\text { Cont. Empl. } \\
\text { (Quarters) }\end{array}$ \\
\hline REBP Region $\times$ Treated Cohort & $\begin{array}{l}0.011^{*} \\
(0.006)\end{array}$ & $\begin{array}{l}0.012^{* *} \\
(0.005)\end{array}$ & $\begin{array}{c}0.062^{* * *} \\
(0.015)\end{array}$ & $\begin{array}{l}-0.018 \\
(0.019)\end{array}$ & $\begin{array}{c}-0.089^{* * *} \\
(0.025)\end{array}$ \\
\hline REBP Region & $\begin{array}{l}-0.003 \\
(0.019)\end{array}$ & $\begin{array}{c}0.008 \\
(0.011)\end{array}$ & $\begin{array}{l}-0.007 \\
(0.056)\end{array}$ & $\begin{array}{c}0.005 \\
(0.041)\end{array}$ & $\begin{array}{c}0.116 \\
(0.088)\end{array}$ \\
\hline Treated Cohort & $\begin{array}{c}0.056^{* * *} \\
(0.004)\end{array}$ & $\begin{array}{c}0.073^{* * *} \\
(0.005)\end{array}$ & $\begin{array}{c}0.288^{* * *} \\
(0.006)\end{array}$ & $\begin{array}{c}0.065^{*} \\
(0.034)\end{array}$ & $\begin{array}{c}-0.227^{* * *} \\
(0.037)\end{array}$ \\
\hline Constant & $\begin{array}{c}0.157^{* * *} \\
(0.051)\end{array}$ & $\begin{array}{l}0.068^{* *} \\
(0.030)\end{array}$ & $\begin{array}{c}0.324^{* *} \\
(0.142)\end{array}$ & $\begin{array}{c}0.136 \\
(0.107)\end{array}$ & $\begin{array}{l}8.166^{* * *} \\
(0.241)\end{array}$ \\
\hline $\begin{array}{l}\text { Observations } \\
\text { Adjusted } R^{2} \\
\text { No of Clusters }\end{array}$ & $\begin{array}{c}178,590 \\
0.006 \\
99\end{array}$ & $\begin{array}{c}178,590 \\
0.015 \\
99\end{array}$ & $\begin{array}{c}178,590 \\
0.011 \\
99\end{array}$ & $\begin{array}{c}178,590 \\
0.002 \\
99\end{array}$ & $\begin{array}{c}178,590 \\
0.003 \\
99\end{array}$ \\
\hline
\end{tabular}

Note: The table reports results of the specification in 5 while dropping all workers who reached retirement age by 1998. REBP captures the effect of REBP-eligibility on the outcomes listed in columns (1) through (5) on a sample of workers employed at the same establishment in May 1988 and February 1994 . The regression specification includes region and cohort effects. Separation denotes an indicator function that is 1 if a worker is not employed by their employer from February 1994 (and May 1988) in February 1996. Separation into Nonemployment denotes an indicator for Separation from the initial employer interacted with an indicator for not being employed in February 1996. Employment Indicator denotes whether a worker is employed in February 1996. Employment (Quarters) , Unemployment (Quarters) and Continuous Employment (Quarters) denote the quarters of employment, unemployment insurance/assistance receipt, and continuous employment with the initial employer between February 1994 and 1996. Standard errors clustered at the administrative region level are reported in parentheses. Levels of significance: ${ }^{*} 10 \%$, ${ }^{* *} \%$, and ${ }^{* * *} 1 \%$. 


\section{F Additional Figures}

Figure A.3: Benefit Extensions and Separations Among Younger Cohorts

(a) Separations (1988 to 1993)

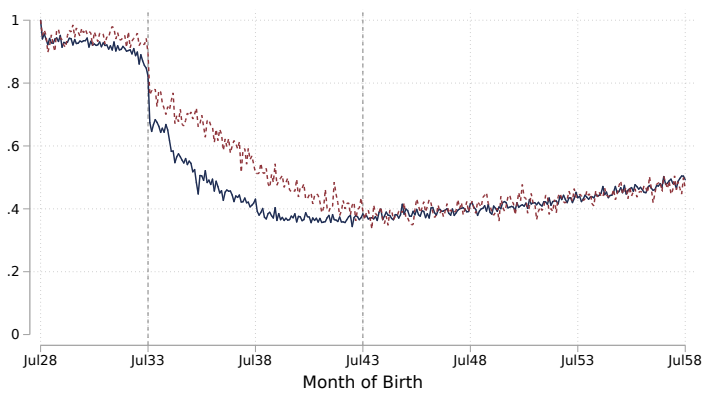

- Separations from 1988 Employer, Non-REBP Region - Separations from 1988 Employer, REBP Region. (b) Difference (REBP - Control Region)

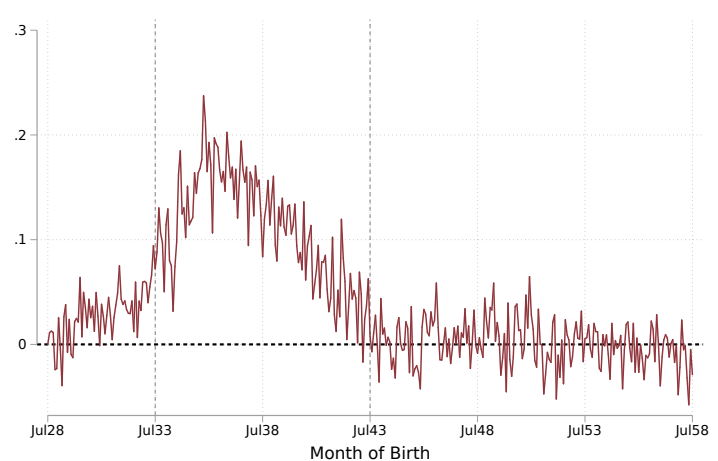

Note: Panel (a) shows the share of workers who separated from their 1988q2-employer (right before the reform) by 1993 q3 (when reform had just ended) for all workers born between 1928 and 1958. It plots rates by month of birth and within the REBP (red, short dashes) and non-REBP (blue, solid) regions. Panel (b) shows the difference between the REBP and the control region by cohort. 
Figure A.4: Benefit Extensions and Employment Outcomes by Age

(a) Quarters Nonemployed (Age 50 to 55)

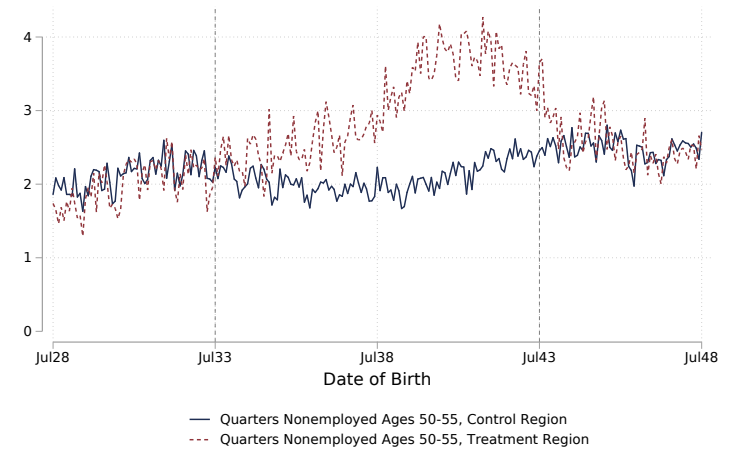

(b) Quarters in Unemp. (Benefits) (Age 50 to 55)

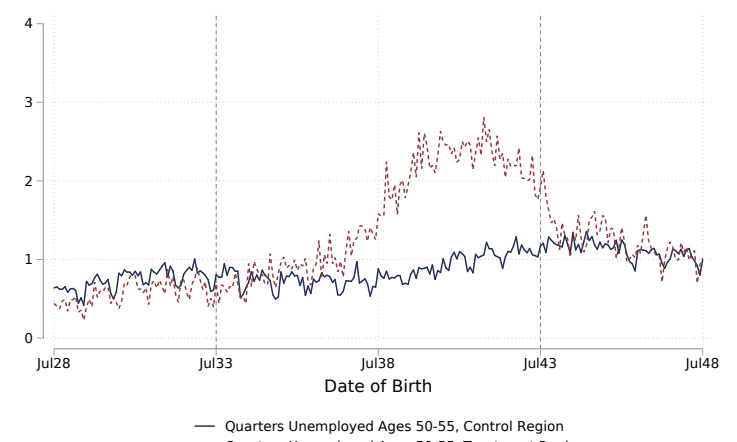

(a) Difference (REBP - Control)

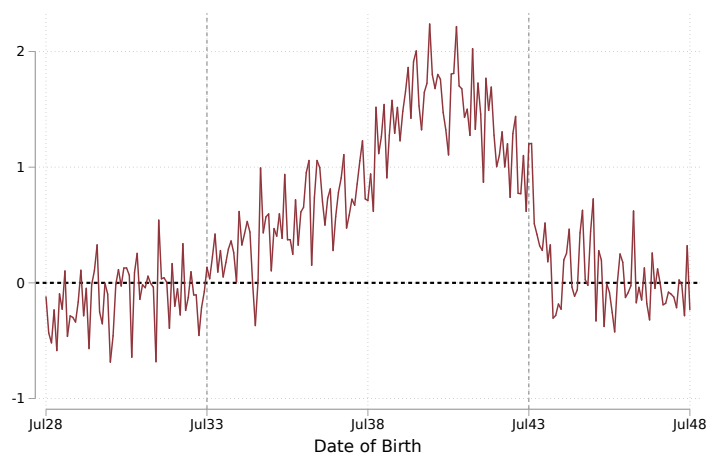

(b) Difference (REBP - Control)

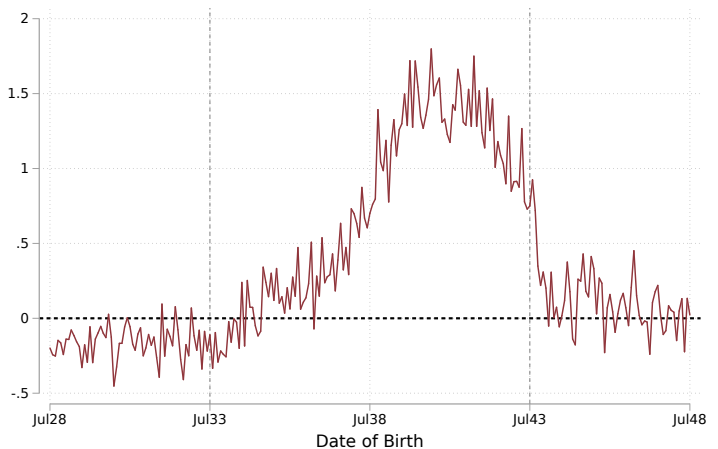

Note: Panels (a) and (c) show the average number of quarters that the workers are employed and on UI, respectively, until the quarter before they turn 55, among those employed in the quarter before they turn 50. Both plot rates by month of birth and within the REBP (red, short dashes) and non-REBP (blue, solid) regions. Panels (b) and (d) show the difference between the REBP and the control region by cohort. Cohorts born after 1943 were not covered by the policy as they turned 50 after the program was abolished 1993. 


\section{Figure A.5: "Phantom" Treatment Effects}

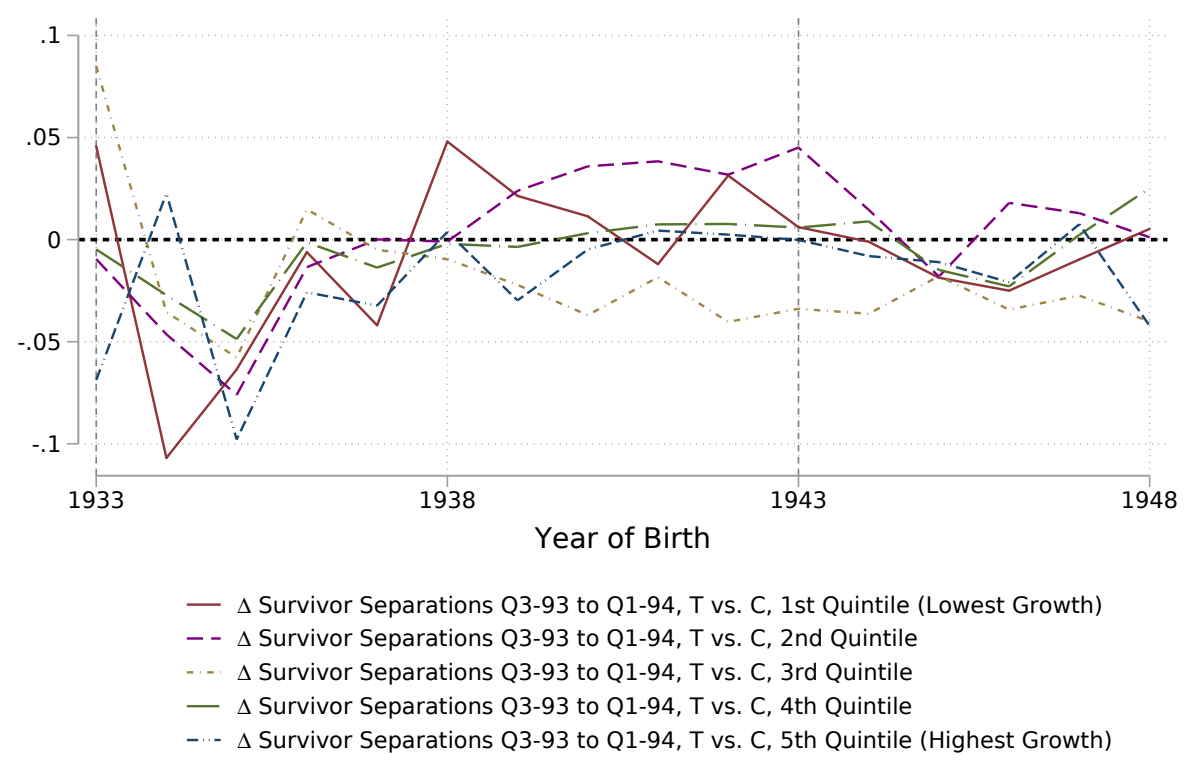

Note: This figure gives a sense of possible post-abolition separation effects by REBP. It plots, by year of birth, the separations from 1993q3, the first quarter after the abolition of the REBP, to 1994q1, among the workers who remain employed in their 1988q2 establishment in 1993q3, i.e. the REBP "survivors." It breaks the separations into quintiles of industry growth rates (not worker-weighted) to emphasize where the additional separations occur. This, along with grandfathering clauses in the law abolishing the REBP (see Section 2), motivates our decision to analyze possible resilience effects by looking at jobs that survived through 1994q1, rather than those surviving only to the abolition of the reform. 


\section{Separations vs. Annual Establishment Growth}

(a) 1994-1995

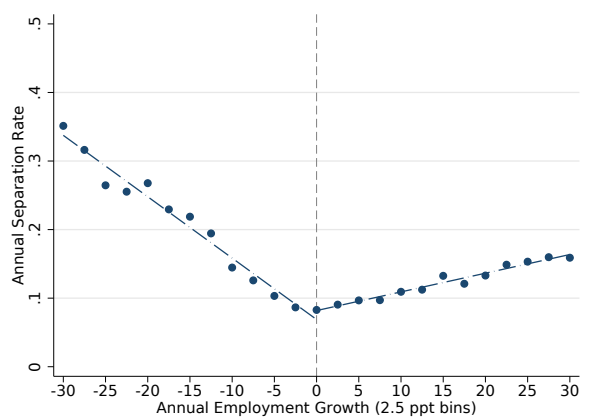

(b) 1994-1997

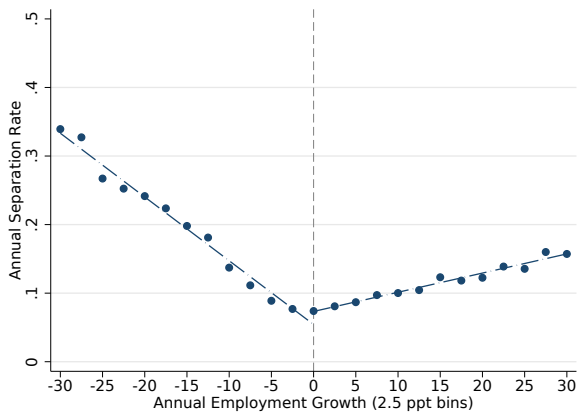

(c) $1994-1998$

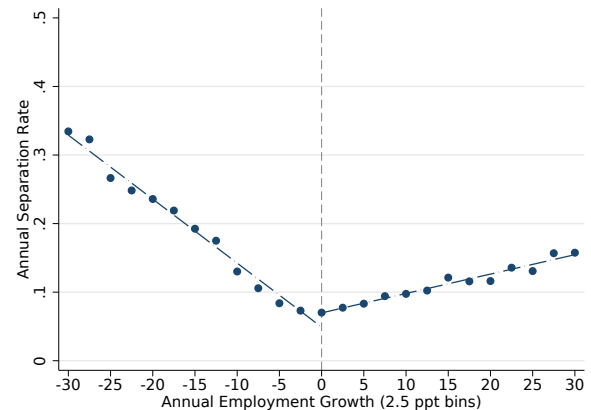

\section{Survivor Separations by Cohort and Region}

(d) 1994-1995

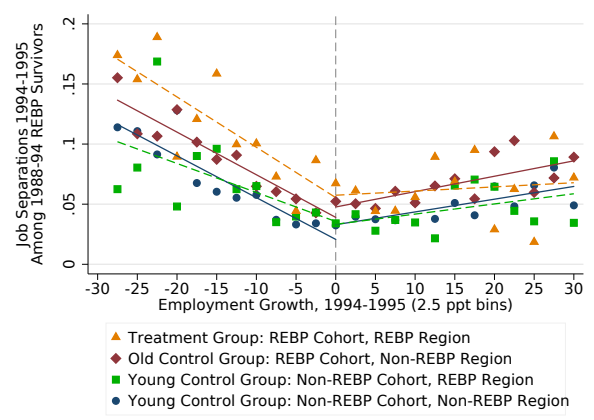

(g) 1994-1995

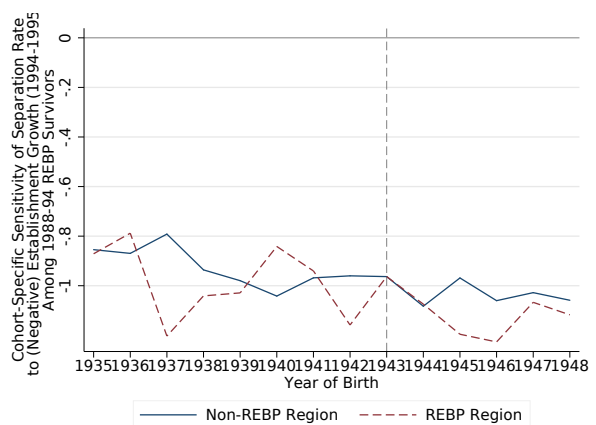

(e) 1994-1997

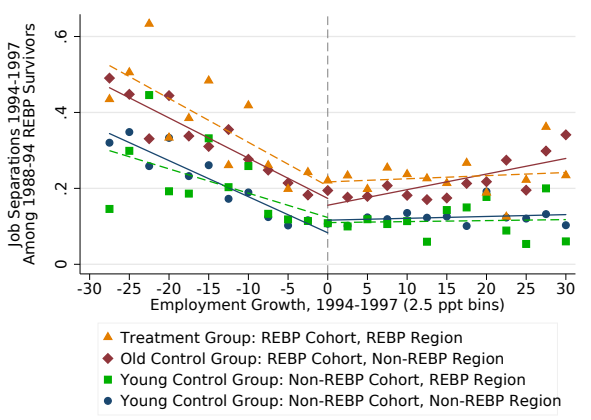

Birth Cohort-Specific Slopes

(h) 1994-1997

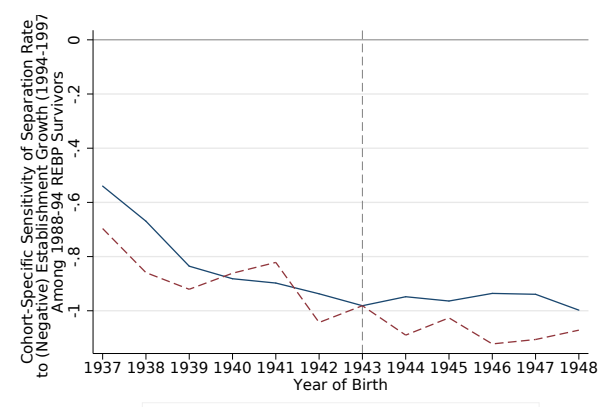

_ Non-REBP Region - - - - REBP Region (f) 1994-1998

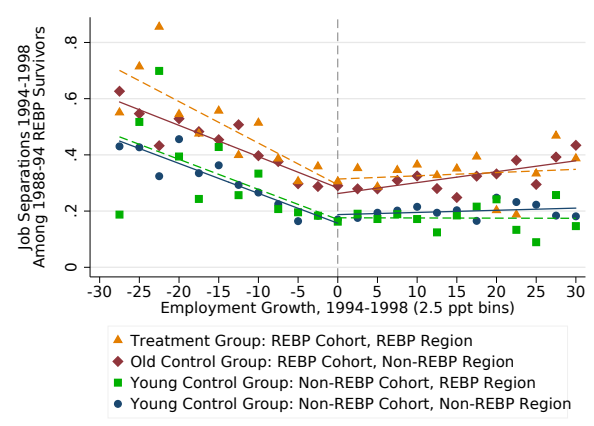

(i) $1994-1998$

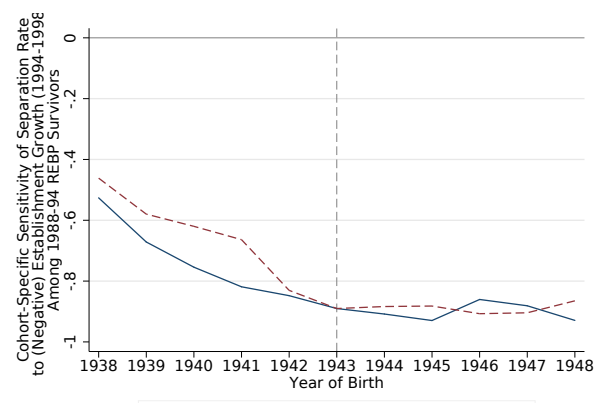

- Non-REBP Region - - --- REBP Region

Note: These figures plot the results of an analysis focusing on labor demand shifts within non-steel sector establishments. In an attempt to confirm the "hockey-stick" relationship between separations and employment growth at the establishment level (Davis et al. 2013) in the Austrian setting, Panels (a), (b), and (c) plot annual separation rates for male workers employed in a given year by bins of annual establishment employment growth rates (first quarter of every year), pooling years 1994 through 1995, 1997, and 1998, respectively. Panels (d), (e), and (f) focus on separations among the four relevant groups tracked throughout the paper: REBP-eligible and -ineligible birth cohorts and regions, while still plotting against total establishment employment growth. We ignore the cohorts born before 1935, 1937, and 1938 since they have reached retirement age in 1995, 1997, and 1998, respectively. Panels (g), (h), and (i) plot the slope of the cohort-specific relationship between separations and establishment growth among shrinking establishments for each year of birth and split between the REBP and non-REBP regions for each time horizon. We adjust throughout for spurious layoffs due to mergers, take-overs, and administrative changes to the ASSD using the procedure outlined in Fink et al. (2010). 


\section{RECENT WORKING PAPERS FROM THE CENTER FOR RETIREMENT RESEARCH AT BOSTON COLLEGE}

Measuring Racial/Ethnic Retirement Wealth Inequality

Wenliang Hou and Geoffrey T. Sanzenbacher, January 2020

A Study of Longitudinal Trajectories of Health and Job Demand on Retirement Age Amal Harrati and David Rehkopf, January 2020

Participation and Pre-Retirement Withdrawals in Oregon's Auto-IRA Laura D. Quinby, Alicia H. Munnell, Wenliang Hou, Anek Belbase, and Geoffrey T. Sanzenbacher, November 2019

Why Are 401(k)/IRA Balances Substantially Below Potential?

Andrew G. Biggs, Alicia H. Munnell, and Anqi Chen, November 2019

How Best to Annuitize Defined Contribution Assets?

Alicia H. Munnell, Gal Wettstein, and Wenliang Hou, October 2019

How Do Older Workers Use Nontraditional Jobs?

Alicia H. Munnell, Geoffrey T. Sanzenbacher, and Abigail N. Walters, October 2019

Will More Workers Have Nontraditional Jobs as Globalization and Automation Spread? Matthew S. Rutledge, Gal Wettstein, and Sara Ellen King, July 2019

Do States Adjust Medicaid Enrollment in Response to Capitation Rates? Evidence from the Medicare Part D Clawback

Laura D. Quinby and Gal Wettstein, June 2019

The Effect of Medicare Part D on Evergreening, Generic Entry, and Drug Prices Geoffrey T. Sanzenbacher and Gal Wettstein, May 2019

Is the Drop in Fertility Due to The Great Recession or a Permanent Change? Alicia H. Munnell, Anqi Chen, and Geoffrey T. Sanzenbacher, March 2019

Will Fewer Children Boost Demand for Formal Caregiving?

Gal Wettstein and Alice Zulkarnain, March 2019

The Relationship Between Occupational Requirements and SSDI Activity

Matthew S. Rutledge, Alice Zulkarnain, and Sara Ellen King, February 2019

How Does Contingent Work Affect SSDI Benefits?

Matthew S. Rutledge, Alice Zulkarnain, and Sara Ellen King, February 2019

All working papers are available on the Center for Retirement Research website (https://crr.bc.edu) and can be requested bye-mail (crr@bc.edu) or phone (617-552-1762). 